\title{
Ajuste de las Variables que Gobiernan los Modelos de Comportamiento de HDM-4 para Vías no Pavimentadas de la Región de Antofagasta - Chile
}

\author{
Oscar Orlando Rojas Cazaluade
}

Tesis presentada para el grado de MAGÍSTER EN INGENIERÍA VIAL

Área Departamental Construcciones

Facultad de Ingeniería

UNIVERSIDAD NACIONAL DE LA PLATA

Diciembre de 2008 
PROFESOR TUTOR

Ing. Juan Manuel Campana

Maestría en Administración (c)

PROFESOR CO-TUTOR

Ing. José Luís Infante

Maestría en Economía (c) 
AGRADECIMIENTOS

A mi familia, sin ellos los objetivos trazados no se hubiesen alcanzado.

A la universidad y compañeros de trabajos que creyeron en esta empresa y supieron esperar.

A Dios, compañero inseparable en mis desafíos. 


\section{Ajuste de las Variables que Gobiernan los Modelos de Comportamiento de HDM-4 para Vías no Pavimentadas de la Región de Antofagasta - Chile}

\section{RESUMEN}

En los últimos veinte años, los sistemas de gestión de carreteras han mejorado significativamente debido al avance logrado en el campo de la informática, permitiendo hacer un mejor uso de los recursos disponibles para el mantenimiento y la rehabilitación de las carreteras.

El programa desarrollado por el Banco Mundial HDM-4 (Highway Development and Management Model), considera modelos de deterioro y conservación de caminos; para pavimentos flexibles, rígidos y no pavimentados.

Respecto a los caminos no pavimentados, el programa hace uso de los modelos de rugosidad y de perdida de material.

En el estudio de Brasil, base del HDM-4, la rugosidad máxima se encontró en función de las propiedades de los materiales y la geometría del camino; la rugosidad mínima corresponde a la cota mínima que puede alcanzar la carpeta de rodado. En tanto la progresión de rugosidad está en función del tiempo y el tránsito.

En tanto la rugosidad mínima corresponde a la cota mínima que se puede alcanzar bajo ciertas condiciones granulométricas de la carpeta de rodado.

La aplicación directa de las expresiones encontradas para Brasil, no representan fielmente lo que acontece en la realidad chilena, por ser un territorio disímil al brasileño, al presentar climas, suelos, topografía y tránsito diferentes, es que se plantea ajustar el modelo a la realidad chilena, en particular para los caminos de Antofagasta.

Los valores encontrados en el ajuste, para la región de Antofagasta de Chile, se obtuvieron con datos recopilados en seguimientos realizados por la Dirección Nacional de Vialidad y con aquellos levantados en terreno durante el periodo de estudio.

La calidad del ajuste realizado es estadísticamente significativa y con un poder explicativo superior a los conseguidos por los modelos de Paterson para Brasil. 
INDICE DE MATERIAS

MATERIAS

Página

CAPITULO I

1.1

INTRODUCCIÓN

Modelo de Rugosidad

Modelo de Pérdida de Material

$1.2 \quad$ PLANTEAMIENTO DEL PROBLEMA 6

1.3 OBJETIVOS 8

1.3.1 Objetivo General 8

1.3.2 Objetivos Particulares 8

1.4 METODOLOGÍA 9

1.5 RESUMEN DE LOS CAPÍTULOS 10

CAPÍTULO II

ANTECEDENTES DE LOS ESTUDIOS DE DETERIORO DE 11

CAMINOS NO PAVIMENTADOS

$2.1 \quad$ INTRODUCCIÓN

2.2 HISTORIA DE LOS MODELOS HDM 12

2.3 ESTUDIO DE DETERIORO EN BRASIL 16

2.3.1 Factores Relevante en el Estudio 17

$\begin{array}{lll}\text { 2.3.2 } & 18\end{array}$

2.3.3 Elección de Modelos de Deterioro 19

2.3.4 Modelo de Variación de Rugosidad en el Tiempo 20

2.3.5 Modelo de Pérdida de Grava en el Tiempo 22

2.3.6 Conclusiones del Estudio Brasileño 24

$2.4 \quad$ VALIDACIÓN DEL MODELO DE DETERIORO - 24

2.4.1 Uso del Modelo de Deterioro HDM 25

2.4.2 Uso del Modelo de Deterioro HDM 26

2.4.3 Conclusiones - Estudio Lorena Araya 26

i) Modelo de Kenya: HDM-II 26

ii) Modelo de Brasil: HDM-III 27

2.5 COMPORTAMIENTO CAMINOS NO PAVIMENTADOS - 29

2.5.1 Resultados de la Medición de la Pérdida de Material 30

2.5.2 Análisis de Rugosidad en los Tramos Testigos 30

i) Rugosidades Mínimas $\quad 30$ 
ii) Rugosidad Máxima 31

iii) Análisis de Rugosidades Antes y Después del 31

2.5.3 Rugosidad en las Estaciones Climáticas 31

2.5.4 Calibración de los Modelos de Deterioro 32

i) Calibración del Modelo de Rugosidad 32

ii) Calibración del Modelo de Reperfilado 34

iii) Calibración del Modelo de Pérdida de Material 35

2.5.5 Conclusión estudio José García 36

i) Tendencias Encontradas en el Estudio 37

ii) Ajuste de los Modelos de Deterioro del HDM - III 39

CAPITULO III $\quad 40$

ESTUDIO DE CAMINOS NO PAVIMENTADOS ESTABILIZADOS 40

CON BISCHOFITA EN LA REGIÓN DE ANTOFAGASTA - CHILE

$3.1 \quad$ INTRODUCCION 40

3.2 OBJETIVO DEL ESTUDIO 40

$3.3 \quad$ AREA DE ESTUDIO 41

3.4 APORTE DE LA BISCHOFITA EN CAMINOS NO 42

3.5 CRITERIO DE SELECCION DE CAMINOS 43

3.5 CRITERIO DE SELECCION DE LOS TRAMOS 46

$\begin{array}{lll}3.6 & \text { DISEÑO DEL ESTUDIO }\end{array}$

3.6.1 Evolución de la Rugosidad 49

3.6.2 Evolución de la Pérdida de Material 49

i) Método de Calicatas $\quad 50$

ii) Método Topográfico $\quad 50$

3.7 VARIABLES MEDIDAS EN EL ESTUDIO 52

3.7.1 Variables Geométricas 52

3.7.2 Variables Geométricas 54

3.7.3 Variables Ambientales: 54

3.7.4 Variables de Tránsito 59

3.8 EQUIPOS E INSTRUMENTOS UTILIZADOS 61

3.8.1 Equipos de Características Geométricas 61

3.8.2 Equipo de Medición de Rugosidad 61

3.8.3 Equipo de medición Características Geotécnicas 64

3.8.4 Equipo de medición de Pérdida de Material 64 
3.9 TOMA DE DATOS 64

3.9.1 Medición de Rugosidad 64

3.9.2 Ensayes de Suelos 65

3.9.3 Mediciones de Pérdida de Material 65

3.9.4 Medición Características Geométricas 65

3.9.5 Almacenamiento de Datos 65

CAPITULO IV 66

ANALISIS DE RESULTADOS $\quad 66$

4.1 INTRODUCCION 66

4.2 CARACTERISTICAS MEDIDAS EN LOS TRAMOS 70

4.2.1 Características Geométricas 70

4.2.2 Características Geotécnicas 72

4.2.3 Tránsito solicitante 74

4.3 ANALISIS PLUVIOMETRICO EN EL AREA DE ESTUDIO 75

4.4 DATOS DE RUGOSIDAD EN LOS TRAMOS TESTIGOS 76

4.5 DATOS DE PÉRDIDA DE MATERIAL EN TRAMOS 87

4.5.1 Valores medidos en tramos del estudio 87

4.5.2 Sumario del análisis de la metodología de medición 88

4.6 ANALISIS DE RUGOSIDAD EN LOS TRAMOS 89

$\begin{array}{lll}\text { 4.6.1 Rugosidad mínima medida en terreno } & 89\end{array}$

4.6.2 Rugosidad máxima medida en terreno 90

4.6.3 Rugosidad media de cada tramo 91

4.6.4 Análisis de rugosidad antes y después de un reperfilado 93

4.6.5 Rugosidad en las estaciones climáticas 93

4.7 AJUSTE DE LOS MODELOS DE DETERIORO 94

4.7.1 Ajuste del modelo de Rugosidad Máxima 94

4.7.2 Ajuste del modelo de Progresión de la Rugosidad 101

4.7.3 Ajuste del modelo de Rugosidad Mínima 110

4.7.4 Ajuste del modelo de pérdida de material 117

$\begin{array}{lr}\text { CAPITULO V } & 119\end{array}$

CONCLUSIONES $\quad 119$

5.1 CONCLUSIONES GENERALES 119

5.1.1 Modelos de rugosidad 119

5.1.2 Modelo pérdida de material 122 
ANEXOS

1. Caminos tipos considerados en el presente estudio

2. Equipo de medición de índice de regularidad

3. Medición de Pérdida de Material por método de calicatas

4. Imágenes varias 
INDICE DE TABLAS

TABLAS

Página

CAPITULO I

Tabla 1.1 : Resumen de la Red Vial de Chile 6

Tabla 1.2 : Caminos no pavimentados por Regiones 7

CAPÍTULO II 11

ANTECEDENTES DE LOS ESTUDIOS DE DETERIORO DE 11 CAMINOS NO PAVIMENTADOS

Tabla 2.1 : Características de los tramos testigos 25

CAPITULO III $\quad 40$

ESTUDIO DE CAMINOS NO PAVIMENTADOS ESTABILIZADOS 40

CON BISCHOFITA EN LA REGIÓN DE ANTOFAGASTA - CHILE

Tabla 3.1 : Caminos no pavimentados de la Región de Antofagasta 45 seleccionados para medir rugosidad

Tabla 3.2 : Caminos no pavimentados de la Región de Antofagasta $\quad 45$ seleccionados para el estudio de pérdida de material

Tabla 3.3 : Tramos elegidos para medir rugosidad 48

Tabla 3.4 : Tramos elegidos para medir pérdida de material 48

Tabla 3.5 : Variables medidas en el estudio 52

Tabla 3.6 : Ubicación de estaciones pluviométricas en el norte de 57 Chile

Tabla 3.7 : Ubicación de estaciones pluviométricas asimiladas al $\quad 57$ estudio

CAPITULO IV

ANALISIS DE RESULTADOS $\quad 66$

Tabla 4.1 : Características geométricas de los tramos 71

Tabla 4.2 : Características geotécnicas de los tramos 73

Tabla 4.3 : Tránsito de los tramos en estudio 74

Tabla 4.4 : Precipitación año 2005 del norte grande 75

Tabla 4.5 : Precipitación año 2006 del norte grande 75

Tabla 4.6 : Precipitaciones totales y promedios de la zona de 76 estudio

Tabla 4.7 : Rugosidad medida en tramo CR1 77

Tabla 4.8 : Rugosidad medida en tramo CR2 77

Tabla 4.9 : Rugosidad medida en tramo CR3 y CR4 78 
Tabla 4.10 : Rugosidad medida en tramo CR5 y CR6 79

Tabla 4.11 : Rugosidad medida en tramo CR7, CR8 y CR9 80

Tabla 4.12 : Rugosidad medida en tramo CR10 y CR11 81

Tabla 4.13 : Rugosidad medida en tramo CR12 y CR13 82

Tabla 4.14 : Rugosidad medida en tramo CR14 y CR15 83

Tabla 4.15 : Rugosidad medida en tramo CR16 y CR17 84

Tabla 4.16 : Rugosidad medida en tramo CR18 y CR19 85

Tabla 4.17 : Rugosidad medida en tramo CR20 y CR21 86

Tabla 4.18 : Pérdida de material - Método calicatas 87

Tabla 4.19 : Pérdida de material (mm) - Método topográfico (mm) 88

Tabla 4.20 : Valores para el análisis de pérdida de material 89

Tabla 4.21 : Rugosidades mínimas $\quad 90$

Tabla 4.22 : Rugosidad máxima $\quad 91$

Tabla 4.23 : Rugosidad media de cada tramo 92

Tabla 4.24 : Correlación entre variable para 21 muestras 95

Tabla 4.25 : Variable dependiente $\mathrm{RI}_{\text {máx }} \quad 96$

Tabla 4.26 : Análisis de la varianza 96

Tabla 4.27 : Determinación rugosidad máxima 99

Tabla 4.28 : Correlación entre variable para 16 muestras 103

Tabla 4.29 : Variable dependiente "c" 104

Tabla 4.30 : Análisis de la varianza 104

Tabla 4.31 : Determinación variable "c" 106

Tabla 4.32 : Valores del parámetro "b" 107

Tabla 4.33 : Cálculo de la variable $\mathrm{RI}_{\mathrm{TG2}} \quad 108$

Tabla 4.34 : Variable dependiente "RImín/D95" 113

Tabla 4.35 : Análisis de la varianza 113

Tabla 4.36 : Modelo rugosidad mínima 115

CAPITULO V 119

CONCLUSIONES 119

GLOSARIO DE TÉRMINOS 125

GLOSARIO SIGLAS 126

REFERENCIAS $\quad 129$

ANEXOS 131 


\section{INDICE DE FIGURAS}

FIGURAS

Página

CAPITULO I

CAPÍTULO II

ANTECEDENTES DE LOS ESTUDIOS DE DETERIORO DE CAMINOS NO PAVIMENTADOS

Figura 2.1 : Componentes que afectan a los usuarios del camino.

Figura 2.2 : Desarrollo de los Modelos HDM.

\section{CAPITULO III}

Figura 3.1 : Zona de caminos en estudio - Región Antofagasta (II)

Figura 3.2 : Caminos no pavimentados, estabilizados con bischofita

- Región Antofagasta

Figura 3.3 : Ubicación geográfica de los caminos testigos

Figura 3.4 : Esquema de ubicación de las calicatas

Figura 3.5 : Esquema de nivelación topográfica

Figura 3.6 : llustración subida y bajadas "RF" y curvatura horizontal

Figura 3.7 : Mapa de Isoyetas de Precipitaciones de la World Meteorological Organization (WMO)

Figura 3.8 : Ubicación de puntos censales de la Región de Antofagasta

Figura 3.9 : Unidad de recolección de datos y acelerómetro

Figura 4.1 : Grafica de la rugosidad máx., mín. y media 93

Figura 4.2 : Gráfica de residuos - $\mathrm{RI}_{\text {máx }} \quad 98$

Figura 4.3 : Comportamiento de la Rugosidad Máxima 100

Figura 4.4 : Modelo ajustado versus modelo de Paterson 100

Figura 4.5 : Gráfica de residuos - "c" 105

Figura 4.6 : Progresión de la Rugosidad 109

Figura 4.7 : Tendencia de los modelos Progresión RI versus lo 109 medido

Figura 4.8 : Tendencia del incremento de la rugosidad 110

Figura 4.9 : Gráfica de residuos - $\mathrm{RI}$ mín 114

Figura 4.10 : Rugosidad Mínima 116 
Figura 4.11 : Modelo $\mathrm{RI}_{\min }$ ajustado versus modelo de Paterson

CAPITULO V

CONCLUSIONES

GLOSARIO DE TÉRMINOS

GLOSARIO SIGLAS

REFERENCIAS

ANEXOS

ANEXO 1: Fotográfico

1. Caminos tipos considerados en el presente estudio

2. Equipo de medición de índice de regularidad internacional - IRI

3. Medición de Pérdida de Material por método de calicatas

4. Imágenes varias 


\section{CAPITULO I}

\subsection{INTRODUCCIÓN}

Los caminos no pavimentados constituyen la mayor proporción de la red de carreteras en Chile y en muchos otros países en vías de desarrollo. La asignación de recursos en los caminos sin pavimentar ha sido realizada históricamente en base a la experiencia de las autoridades de carreteras.

La red vial existente en la actualidad en Chile, está compuesta por aproximadamente un tercio de caminos con carpetas pavimentadas, ya sea en hormigón o asfalto. Constituyendo éstas la red que nos permite satisfacer las demandas de transporte para los viajes interregionales e internacionales, e incluso da respuesta a la generación de viajes, que en el día a día, se materializan en las grandes ciudades. Los dos tercios restante de la red, permanecen con carpetas de ripio, o simplemente de tierra, las que se distribuyen en su mayoría en la red primaria y secundaria, que abarca a un sector un tanto más postergado por su lejanía a la ciudad, y que, dado su emplazamiento, tiene a estos caminos como arteria principal que permite su abastecimiento, desarrollo comercial, y la posibilidad cierta de optar a los centros educacionales y de libre esparcimiento.

Actualmente en Chile está en desarrollo el programa "Caminos Básicos 5.000", iniciativa con la cual se espera contribuir al desarrollo del país, a la superación de la pobreza y apoyar la economía productiva rural. Este gran desafío, que fue dado a conocer públicamente en agosto 2003 y se estima que concluya el presente año, para luego iniciar nuevos programas viales en dicha dirección. El programa ha sido calificado por el Director Nacional de Vialidad, como la "Segunda Revolución Vial en Chile", ya que la primera sería la desarrollada por el sistema de concesiones. El mejoramiento de caminos rurales no pavimentados, mediante soluciones básicas y una eficiente gestión de los recursos, es una forma de acercar el progreso en infraestructura del país a aquellas zonas rurales más apartadas.

El hecho de habilitar de mejor forma los caminos de zonas rurales implica un 
alto impacto social, ya que permite potenciar la conectividad en lugares aislados, como asimismo, elevar la calidad de vida de sus habitantes.

La alta inversión requerida no permite mejorar la conectividad de las comunidades que dependen de estos caminos mediante proyectos de alto estándar debido a su baja rentabilidad, por tanto la consigna es poder "hacer más con menos".

En los últimos veinte años, los sistemas de gestión de carreteras han mejorado significativamente debido al avance logrado en el campo de la informática. Hoy los administradores de carreteras disponen de una serie de herramientas o mecanismos que le permiten hacer un mejor uso de los recursos disponibles para el mantenimiento y la rehabilitación de las carreteras.

El HDM-4 fue desarrollado como parte del "International Study of Highway Development and Management Tools" (ISOHDM), proyecto internacional para desarrollar nuevos mecanismos de análisis de inversión, que contó con el patrocinio del Banco Mundial, la AIPCR, el Departamento de Desarrollo Internacional del Reino Unido, El Banco de Desarrollo Asiático y la Administración Nacional de Caminos Sueca entre otros ${ }^{1}$.

En la actualidad la Dirección de Vialidad de Chile utiliza, para la asignación de los limitados recursos de la red vial, los modelos computacionales Highway Development and Management Model (HDM-4) y Expediture Budgeting Model (EBM), los cuales permiten evaluar económicamente los proyectos viales y asignar recursos en forma óptima. Los modelos de deterioro que se aplican en Chile se basan en el análisis del estudio de Brasil ${ }^{2}$.

El programa desarrollado por el Banco Mundial HDM-4, contempla modelos de Deterioro y Conservación de Caminos; tanto para caminos con pavimentos flexibles, rígidos y aquellos no pavimentados. Respecto a los caminos no

\footnotetext{
${ }^{1}$ PIARC. (2005). Página Web oficial de la "World Road Association" (Asociación Mundial de Carreteras). http://www.piarc.org/es.

${ }^{2}$ William Paterson D.O. (1987). "Road Deterioration and Maintenance Effects: Models for Planning and Management. Highway Desing and Maintenance Standards Series", World Bank Transportation department, Washington D.C.
} 
pavimentados, el programa hace uso de los modelos de rugosidad y de perdida de material.

\section{Modelo de Rugosidad}

El modelo de rugosidad se fundamenta en el perfil del camino por donde transita la rueda, puesto que este incide en los costos de operación del vehículo. La ubicación de la huella tiende a variar cuando la rugosidad alcanza niveles altos, ya que los vehículos buscan minimizar el impacto dinámico; la predicción de la progresión de la rugosidad debe tener en cuenta esta tendencia autorreguladora. A causa de la alta variabilidad de las propiedades de los materiales, drenaje, erosión de la superficie y los altos niveles de rugosidad de los caminos no pavimentados, los errores de la predicción tienden a ser grandes, el error estándar del estudio brasileño es del orden de 1,5 a 2,5 $\mathrm{m} / \mathrm{km}$ IRI, para un nivel del confianza del 95\%.

Como se ha enunciado, los modelos de rugosidad, corresponden por un lado al de Progresión de la Rugosidad (variación de rugosidad en el tiempo) y al Efecto de Reperfilado (mejoramiento de la condición superficial luego de las acciones de conservación).

Los submodelos de la rugosidad son:

- Rugosidad máxima

- Progresión de la rugosidad

- Rugosidad mínima

- Efecto de la compactación en la progresión de la rugosidad.

- Efecto del reperfilado (graduación)

- Rugosidad media durante el año de análisis

- Ciclo de la rugosidad - estado estable -

El modelo adoptado por el HDM circunscribe a la rugosidad a un límite superior 
o rugosidad máxima $\left(R I_{\max }\right)$ desde la cual una función convexa decreciente con tasa exponencial inversa desciende hasta el valor esperado. En el estudio de Brasil $^{3}$, la rugosidad máxima $\left(R I_{\max }\right)$ se encontró en función de las propiedades de los materiales y la geometría del camino, mientras que la proporción de progresión de rugosidad está en función de la rugosidad en un tiempo determinado, la rugosidad máxima, el tiempo, el tránsito (vehículos livianos y pesados) y las propiedades de los materiales.

Respecto a las actividades de mantenimiento en caminos sin pavimentar, generalmente se realiza varias veces al año, cada una tendiente a reducir los niveles de rugosidad. En el estudio se encontró que la magnitud de reducción de la rugosidad, depende de la rugosidad antes de las actividades de mejoramiento, de las propiedades de los materiales y de la rugosidad mínima. El modelo del efecto de Reperfilado, nace de la condición de rugosidad más baja que puede alcanzar el camino $\left(R I_{\text {minn }}\right)$ y sobre la cual las actividades de mantenimiento no tienen mayor efecto.

La relación para predecir la rugosidad después del reperfilado se expresa como una función lineal de la rugosidad antes de las actividades de mantenimiento, de la relación de polvo y la rugosidad mínima.

\section{Modelo de Pérdida de Material}

Regravillado es la acción de mantenimiento mayor (principal) en los caminos no pavimentados, análogo en importancia a la acción de recubrir un camino pavimentado, así, la frecuencia requerida es una importante decisión de la planificación. La pérdida de material se define durante un período de tiempo como el cambio en el espesor de la capa granular y se usa para estimar cuando el espesor de esta capa, por desgaste, ha disminuido su espesor a un nivel dónde el regravillado es necesario. Paterson identificó tres factores que afectan la capa granular, incidiendo en la pérdida de material: desgaste, tráfico y la influencia de la graduación del material. Las propiedades del materiales, la

\footnotetext{
${ }^{3}$ William Paterson D.O. (1987). "Road Deterioration and Maintenance Effects: Models for Planning and Management. Highway Desing and Maintenance Standards Series", World Bank Transportation department, Washington D.C.
} 
alineación del camino y el ancho del camino, influyen directamente en la perdida de material de la capa granular de rodado. La relación para predecir la pérdida del material anual de la capa de rodadura, se desarrollo en función de la lluvia mensual, del volumen de tráfico, la geometría del camino y de las características del material granular.

En definitiva, los modelos de deterioro de los caminos no pavimentados, estiman el deterioro de la superficie en función del tipo de pavimento, tránsito, clima, geometría, tipo de material y de las políticas de conservación que se apliquen a los mismos.

Los modelos HDM son usados en más de 60 países en diferentes tipos de estudios de inversión. Predice la actuación del pavimento en el tiempo, bajo el efecto del tráfico y de las acciones de mantenimiento.

El programa desarrollado por el Banco Mundial para apoyar a los profesionales que se desempeñan en el ámbito de la administración de carreteras, incorpora modelos de comportamiento de estructuras de pavimentos que deben ser ajustados a las condiciones particulares de cada país o región donde serán utilizados.

La importancia del proceso de ajustar los modelos de comportamiento está en el impacto económico, ya que debido a la influencia directa en el inicio y progresión de los deterioros del camino, se hace necesaria una adecuada adaptación de ellos, de modo que se pueda efectuar una evaluación económica certera de los proyectos viales.

Este trabajo de Tesis tendrá como objetivo ajustar los modelos de deterioro utilizados en la actualidad por la Dirección de Vialidad de Chile en los caminos no pavimentados de la región de Antofagasta, específicamente los estabilizados con bischofita, los cuales están presente en el programa “Caminos Básico 5000". Los modelos que se ajustarán son los siguientes:

- Rugosidad máxima - $\mathrm{RI}_{\max }$

- Progresión de la rugosidad - $\mathrm{RI}_{\mathrm{TG} 2}$ 
- Rugosidad máxima - $\mathrm{RI}_{\min }$

- Pérdida de material - MLA

\subsection{PLANTEAMIENTO DEL PROBLEMA}

En atención a que la investigación se realizará sobre caminos chilenos, es importante describir en general el inventario de la red de carreteras de Chile y en particular la red de caminos de la Región de Antofagasta.

La tabla 1.1 entrega un resumen con información de las características más relevantes de la red vial de responsabilidad del Ministerio de Obras Públicas MOP, a diciembre del 2004, cuyo detalle está contenido en el documento "Red Vial Nacional, Dimensionamiento y Características - Dic. 2004", que edita el Departamento de Gestión Vial de la Subdirección de Desarrollo de la Dirección de Vialidad ${ }^{4}$.

Tabla 1.1: Resumen de la Red Vial de Chile

\begin{tabular}{|c|l|c|c|}
\hline Ítem & \multicolumn{1}{|c|}{ Detalle } & $\begin{array}{c}\text { Longitud en } \\
\text { kilómetros }\end{array}$ & $\begin{array}{c}\text { Distribución } \\
\text { Porcentual } \\
\text { (\%) }\end{array}$ \\
\hline 1 & Longitud Total Red Vial & $80.672,33$ & 100 \\
\hline 2 & Longitud Red Pavimentada & $16.785,85$ & 20.8 \\
\hline 3 & Red Vial Solución Básica & $5.163,68$ & 6,4 \\
\hline 4 & Longitud Red Vial No Pavimentada & $58.722,80$ & 72.8 \\
\hline 5 & Longitud de la Red Vial con Doble Calzada & $2.285,13$ & 2,8 \\
\hline 6 & Longitud de la Red Vial Concesionada & $2.138,16$ & 2,7 \\
\hline 7 & Longitud total de 22 Túneles & 26,33 & \\
\hline 8 & Longitud total de 416 Pasarelas (luz libre) & 18,14 & \\
\hline 9 & Longitud total de 47 Ciclovías & 179,18 & \\
\hline
\end{tabular}

Como se observa, la Red Vial Chilena tiene 80.672 kilómetros de caminos, de los cuales 63.886 kilómetros corresponden a caminos no pavimentados, y de

\footnotetext{
${ }^{4}$ MOP. (Ministerio de Obras Públicas) (abril 2005). Dirección de Vialidad. Subdirección de Planificación y Estudios. Informe anual "Red Vial Nacional Dimensionamiento y Características".
} 
estos, 4.670 kilómetros han recibido alguna de las soluciones del programa Caminos Básico 5000. Lo que significa que el $79 \%$ de la red total son caminos no pavimentados. De aquí la importancia de realizar una buena conservación de ellos y de continuar con el programa de gobierno Caminos Básicos 5000.

El deterioro de los caminos no pavimentados se caracteriza principalmente por su rugosidad y por la perdida de material de la superficie. Las relaciones de predicción para estos dos parámetros se basan en el análisis del estudio del HDM-4 de Brasil.

Tabla 1.2: Caminos no pavimentados por Regiones

\begin{tabular}{|c|c|c|c|c|}
\hline \multirow{2}{*}{ Región } & \multicolumn{3}{|c|}{ Red Vial No Pavimentada (en km.) } & \multirow{2}{*}{$\begin{array}{c}\text { Total } \\
\text { (en km.) }\end{array}$} \\
\hline & $\begin{array}{c}\text { Sol. Básica } \\
\text { (asfalto, sal, } \\
\text { bischofita, otras) }\end{array}$ & Ripio & Tierra & \\
\hline I & 246,05 & 417,07 & $2.842,56$ & $3.505,68$ \\
\hline II & 328,03 & 997,84 & $3.353,60$ & $4.679,47$ \\
\hline III & $1.436,08$ & 826,10 & $3.543,83$ & $5.806,01$ \\
\hline IV & 240,21 & $2.884,46$ & $1.327,76$ & $4.452,43$ \\
\hline V & 822,14 & 686,30 & 425,45 & $1.933,89$ \\
\hline VI & 307,42 & $1.563,49$ & $1.204,57$ & $3.075,48$ \\
\hline VII & 494,70 & $3.764,85$ & $1.993,93$ & $6.253,48$ \\
\hline VIII & 142,40 & $5.186,18$ & $1.948,08$ & $7.276,66$ \\
\hline IX & 282,50 & $7.706,85$ & $2.544,31$ & $10.533,66$ \\
\hline$x$ & 206,90 & $8.271,25$ & 834,70 & $9.312,85$ \\
\hline$X I$ & 62,70 & $2.605,48$ & 227,76 & $2.895,94$ \\
\hline XII & 172,72 & $2.318,08$ & 362,71 & $2.853,51$ \\
\hline R.M. & 421,83 & 885,59 & 393,53 & $1.307,42$ \\
\hline Total & $5.163,68$ & $38.113,54$ & $20.609,26$ & $63.886,48$ \\
\hline
\end{tabular}

En tanto, la tabla 1.2 corresponde a un extracto de la red de caminos no pavimentados por regiones, de responsabilidad del MOP y que se encuentra contenida en el documento "Red Vial Nacional, Dimensionamiento y Características - Dic. 2004". En lo importante, se detallan los caminos de tierra, ripio y aquellos caminos no pavimentados insertos en el programa Caminos 
Básicos 5000, que han recibido soluciones básicas de estabilización, como es el caso de las carpetas granulares estabilizadas con bischofita en la región de Antofagasta.

\subsection{OBJETIVOS}

Asumiendo que la aplicación directa de las expresiones encontradas para el caso brasileño, no representan fielmente lo que acontece en la realidad chilena y sus regiones, por ser un territorio muy disímil al brasileño, además de presentar en toda su extensión diferentes zonas climáticas, tipos de suelos, topografía, tránsito, etc., es que se plantea la necesidad de ajustar el modelo HDM-4 a la realidad chilena, en particular para los caminos estabilizados con bischofita de la Región de Antofagasta.

\subsubsection{Objetivo General}

El objetivo principal de la investigación es ajustar los modelos de comportamiento de caminos no pavimentados en HDM-4, específicamente los modelos de rugosidad y pérdida de material.

Con respecto a la progresión de la rugosidad, el modelo existente predice esta variable con valores que escapan de los resultados empíricos existentes, siendo necesario efectuar un ajuste de los actuales modelos. Para tal efecto, se utilizarán mediciones existentes de rugosidad y pérdida de material, las consideraciones y recomendaciones efectuadas a los modelos brasileños expuestos en los estudios hasta hoy realizados, y las actividades de conservación de la Dirección Regional de Vialidad de Chile.

\subsubsection{Objetivos Particulares}

En tanto que los objetivos particulares que se persiguen en este estudio son los siguientes:

- Comprender los fenómenos que determinan el comportamiento de un camino no pavimentado en el tiempo y la sensibilidad de la condición del camino que se observa ante la incidencia de determinados factores en el 
inicio y progresión de los deterioros que se presentan típicamente en este tipo de estructuras.

- Entender la influencia de cada uno de los componentes de las ecuaciones que conforman los modelos de comportamiento de caminos no pavimentados y la sensibilidad de cada una de las variables que actúan en los deterioros estudiados.

- Determinar las variables y parámetros de mayor influencia en la obtención de factores óptimos de ajuste.

\subsection{METODOLOGÍA}

Para alcanzar los objetivos planteados, se propuso la siguiente metodología:

- Recopilación de antecedentes bibliográficos (desarrollo de los modelos de comportamiento del HDM en el mundo) y de la base de datos de la Dirección de Vialidad de Chile información referida a la región de Antofagasta, respecto de la estructura de los caminos no pavimentados, tránsito, clima, índice de estado, conservación, etc.

- Con los instrumentos que posee Vialidad se desarrolló una metodología similar a la realizada en Brasil para medir tanto la rugosidad como la perdida de material.

- Se definió como zona de testigo a los caminos de la Región de Antofagasta.

- Se midió la evolución de rugosidad y pérdida de material.

- Con los resultados obtenidos se calibraron los modelos de deterioro dados por el HDM-4

- Se analizaron los resultados obtenidos con los modelos originales y los propuestos.

- Finalmente, se plantean las conclusiones generales a las que se arribó en el estudio. 


\subsection{RESUMEN DE LOS CAPÍTULOS}

El Capítulo I corresponde a la presente introducción, que contiene el planteamiento del problema, los objetivos, la metodología y el resumen de los capítulos.

El Capítulo II entrega una recopilación de los últimos estudios referente al deterioro de caminos no pavimentados realizados en Chile y el experimento brasileño.

En el Capítulo III se detalla el presente estudio, explicando el diseño y la ejecución de la experiencia.

El Capítulo IV corresponde al análisis de las variables y a la calibración de los modelos de deterioro que incluye el HDM-4. En este punto, considerando los resultados encontrados en la etapa anterior, y los datos con que se dispone en la actualidad, se procederá a ajustar el modelo de rugosidad existente, comparándose los resultados de la nueva modelación con la propuesta por el modelo brasileño.

Finalmente, en el Capítulo $\mathrm{V}$ se plantean las conclusiones generales a la que se arribó en el estudio, así como también recomendaciones pertinentes para futuras investigaciones. 


\section{CAPÍTULO II}

\section{ANTECEDENTES DE LOS ESTUDIOS DE DETERIORO DE CAMINOS NO PAVIMENTADOS}

\subsection{INTRODUCCIÓN}

Como ya comentáramos en el capítulo anterior, los caminos no pavimentados constituyen la mayor proporción de la red de carreteras en Chile y en muchos otros países en vías de desarrollo e históricamente la asignación de recursos para estos caminos ha sido en base a la experiencia de los administradores de carreteras, siendo para el caso de Chile la Dirección de Vialidad.

En la actualidad la Dirección de Vialidad utiliza, para la evaluación económica de la red vial, el modelo de deterioro HDM en su tercera versión, que posee las relaciones de costo de operación de vehículos derivadas de los estudios de Kenya, Caribe, Brasil e India, así como las relaciones de deterioro obtenidas fundamentalmente del estudio desarrollado en Brasil; y en el presente se encuentra implementando la versión HDM-4.

La Dirección de Vialidad del Ministerio de Obras Públicas, a cargo de la administración de la red vial pública nacional, a través de su Unidad de Gestión Vial, ha venido desarrollando desde comienzo de la década de los ochenta, investigaciones sistemáticas al comportamiento de los pavimentos.

Estructuras de pavimentos flexibles se han trabajado en conjunto con la Pontificia Universidad Católica de Chile para ajustar los modelos de deterioro del programa HDM-III, obteniéndose valores de los factores de adaptación local para distintas condiciones climáticas, de tránsito y de capacidad estructural, principalmente ${ }^{1}$.

En tanto que en estructuras de caminos de tierra y de ripio, denominados caminos de bajo tránsito, la Dirección Nacional de Vialidad en conjunto con el

\footnotetext{
${ }^{1}$ Videla, C.; De Solminihac, H.; Gaete, R.; Bustos, M. (1996), Ajuste de Factores de Calibración para Ampliar Modelos de Deterioro de Pavimentos Asfálticos. Ministerio de Obras Públicas y Pontificia Universidad Católica de Chile.
} 
IDIEM (Centro de Investigación, Desarrollo e Innovación de Estructuras y Materiales) de la Universidad de Chile, desarrollaron la investigación "Análisis de los Modelos de Deterioro para Caminos no Pavimentados Incluidos en el HDM-III y Proposición de Umbrales de rugosidad para Evaluaciones Económicas" ${ }^{2}$, basada fundamentalmente en los estudios desarrollados por Lorena Araya Carvajal $^{3}$ en el año 1988 y José Antonio García Aranzaes ${ }^{4}$ en el año 1993.

Este capítulo tiene como objetivo entregar los antecedentes históricos y básicos de los estudios realizados sobre la materia, deteniéndose en el de Brasil por su mayor importancia y en Chile, a los desarrollados por Lorena Araya Carvajal y José Antonio García por ser más explícitos y encontrarse en la dirección de la presente investigación.

\subsection{HISTORIA DE LOS MODELOS HDM}

La relación entre los estándares de diseño de las carreteras, su condición y los costos de operación de los vehículos ha sido por mucho tiempo de interés de los ingenieros de caminos.

El costo de transporte, a menudo llamado "costo de operación del vehículo (VOC)" o "efectos del camino en los usuario (RUE)", es utilizado para modelar y apreciar los efectos medioambientales y económicos sobre los usuarios del camino.

EI VOC refleja los componentes específicamente asociado con el funcionamiento del vehículo; en tanto que el RUE reflejar los otros componentes que afectan a los usuarios de los caminos, figura 2.1.

Mientras la mayoría de las primeras investigaciones del costo de operación de los vehículos se dirigió en los países desarrollados, particularmente en los

\footnotetext{
${ }^{2}$ IDIEM - Vialidad. (2000). Informe final "Análisis de los Modelos de Deterioro para Caminos no Pavimentados Incluidos en el HDM-III y Proposición de Umbrales de rugosidad para Evaluaciones Económicas"

${ }^{3}$ Araya C., Lorena (1988). "Validación del Modelo de Deterioro Brasileño de Caminos no Pavimentados. Aplicación al caso Chileno".

4 García A., José (1993). "Estudio del Comportamiento de Caminos no Pavimentados de la Zona Central de Chile".
} 
Estados Unidos de América, había una necesidad creciente por conocer las apreciaciones económicas en los países en vías de desarrollo.

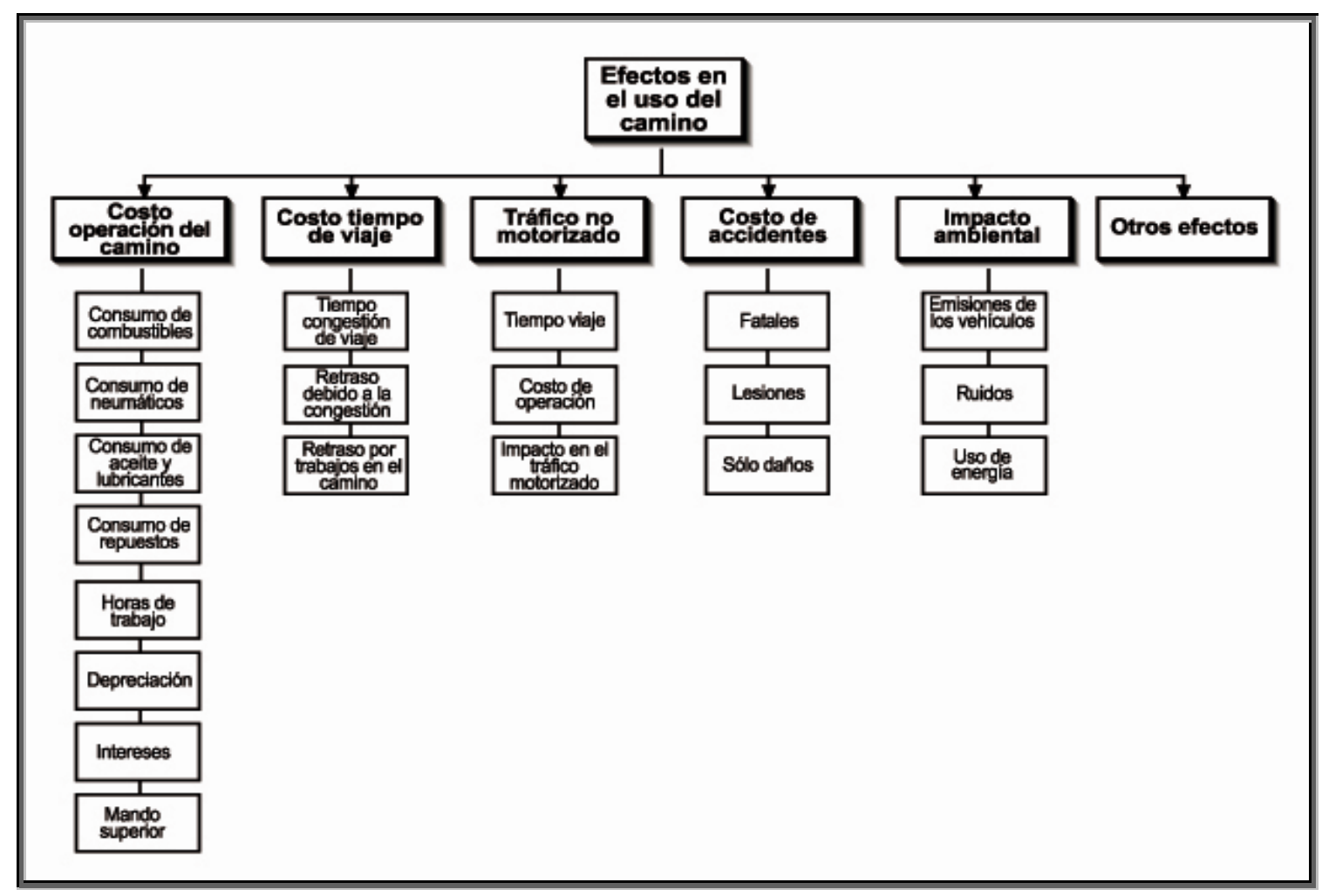

Figura 2.1: Componentes que afectan a los usuarios del camino.

Inicialmente, los análisis económicos de carreteras se dirigieron a minimizar los costos de construcción. Con la publicación de la información de los costos de operación de los vehículos, era posible determinar los costos de operación asociados con algún diseño en particular. Pero no había ningún sustento para considerar la interrelación entre las normas de construcción, de mantenimiento y el costo de operación de los vehículos.

En 1969 el Banco Mundial comenzó un programa para investigar esta interrelación con respecto a los caminos de bajo volumen de tránsito.

La primera fase del estudio se completo en 1971, qué emprendió un grupo de investigación del Instituto Tecnológico de Massachusetts (MIT). Quienes desarrollaron una base conceptual para relacionar la construcción y el mantenimiento a los costos de operación de los vehículos. El objetivo era determinar un conjunto de normas que minimizaran los costos totales del 
transporte. Se concluyó de este estudio inicial que faltaba evidencia empírica legítima en muchas de las relaciones de costo que eran necesarias para determinar el modelo económico y las estrategias de mantenimiento.

Los resultados del principal estudio de campo emprendido entre los años 197175 en Kenya, que investigó pavimentos y el deterioro de caminos no pavimentados, así como los factores que afectan los costos de operación de los vehículos ${ }^{5}$, se usaron como base por el Transport and Road Research Laboratory - TRRL para desarrollar Modelos de Inversión del Transporte Caminero (RTIM), evaluando los costos totales del transporte para una sola ruta ${ }^{6}$.

El HDM - The Highway Design and Maintenance Standards Model (Desarrollo de Modelos de Gerenciamiento de Carreteras) se desarrolló en el año 1977, incorporando las características de los modelos RTIM y MIT. En el año 1981 presenta al mercado la segunda versión del modelo $\mathrm{HDM}^{-1 I^{7}}$ y en 1987 se presenta una nueva actualización de la versión del modelo HDM-III ${ }^{8}$.

En la figura 2.2 se muestran las distintas etapas del desarrollo de HDM desde sus inicios. Desde 1987 se han venido realizado variados y complejos mejoramientos a los nuevos modelos desarrollados, desde una versión simple para computadoras personales hasta modelos más especializados que usan los componentes de HDM-III para predecir los costos de operación de los vehículos y los de deterioro del pavimento.

No obstante, el HDM-III se haya aplicado en más de 100 países, se le reconocen limitaciones en las relaciones entre el costo de operación de los

\footnotetext{
${ }^{5}$ Hodges, J.W., Rolt, J. and Jones, T.E. (1975). The Kenya Road Transport Cost Study: Research on Road Deterioration. TRL Report LR 673, Department of the Environment, Crowthorne.

${ }^{6}$ Robinson, R., Hide, H., Hodges, J.W., Rolt, J. and Abaynayaka, S.W. (1975). A Road Transport Investment Model for Developing Countries. TRL Report LR 675, Department of the Environment, Crowthorne.

${ }^{7}$ Watanatada, T. (1981). Highway Design and Maintenance Standards Model (HDM) Model Description and User's Manual - Release II. Transportation, Water and Telecommunications Department Report, the World Bank, Washington, D.C.

${ }^{8}$ Watanatada T, Harral C G, Paterson W D O, Dhareshwar A M, Bhandari A, and Tsunokawa K, (1987), The Highway Design and Maintenance Standards Model, Volume 1 - Description, The World Bank, John Hopkins University Press.
} 
vehículos y el deterioro de los pavimento, no logrando modelar todos los efectos del camino sobre los usuarios.

Esto llevó a ISOHDM - International Study of Highway Development and Management Tools (Estudio Internacional de Herramientas para el Desarrollo y Administración de Carreteras) que puso en práctica y actualizó el HDM-III desde 1993 al 2000, a desarrollar el HDM-4, versión que también ha estado actualizándose desde su puesta en servició a la fecha.

El HDM-4 fue parte del proyecto internacional para el desarrollo de nuevos mecanismos de análisis de inversión, que contó con el patrocinio del Banco Mundial, la AIPCR, el Departamento de Desarrollo Internacional del Reino Unido, El Banco de Desarrollo Asiático y la Administración Nacional de Caminos Sueca entre otros.

Los modelos de deterioro de caminos HDM-4 para caminos no pavimentados, están basados en las especificaciones del HDM-III estudiadas por Watanatada $(1987)^{9}$. Se han agregado modificaciones menores en el texto y se han incorporando los factores de calibración de los modelos para facilitar la calibración y adaptación local. Los aspectos de fondo del modelo están dados por Paterson ${ }^{10}$.

El nuevo HDM-4, comparado con su predecesor (HDM-III), abarca un rango más amplio de necesidades de las agencias de transportes, instituciones internacionales de financiamiento, consultores e institutos de investigación. Esto se ha logrado a través del desarrollo de herramientas independientes que permiten realizar las siguientes funciones:

- Planificación estratégica

- Elaboración de programas de trabajo

\footnotetext{
${ }^{9}$ Watanatada et al. (1987), The Highway Design and Maintenance Standards Model, Volume 1 - Description, The World Bank, John Hopkins University Press.

${ }^{10}$ William Paterson D.O. (1987). "Road Deterioration and Maintenance Effects: Models for Planning and Management. Highway Desing and Maintenance Standards Series", World Bank Transportation department, Washington D.C.
} 
- Preparación de proyectos

- Investigación y estudio de políticas

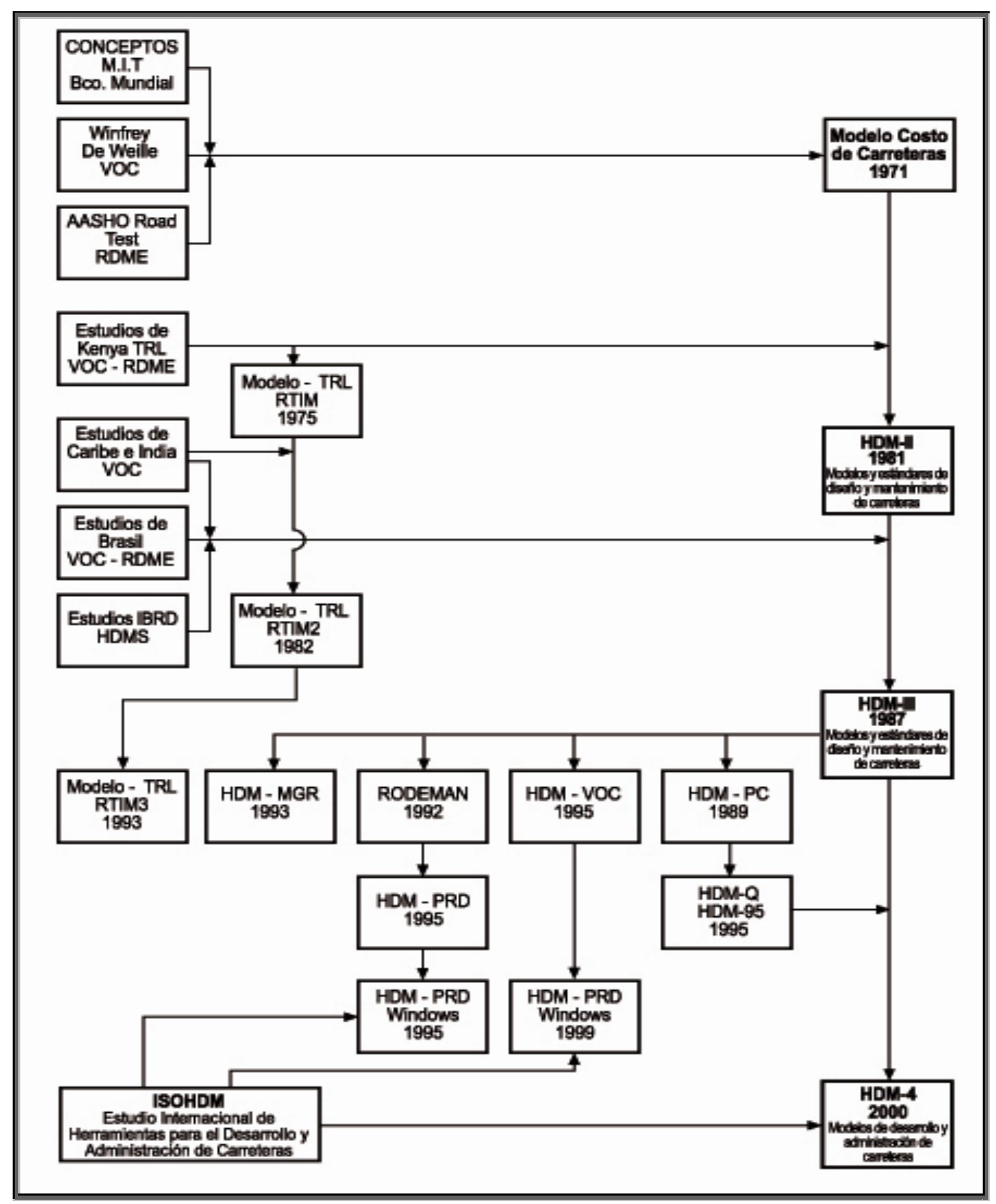

Figura 2.2: Desarrollo de los Modelos HDM.

\subsection{ESTUDIO DE DETERIORO EN BRASIL}

En enero de 1975 se acordó entre el Gobierno de Brasil y el Programa de Naciones Unidas para el Desarrollo (PNUD), analizar y desarrollar métodos y 
modelos tendientes a minimizar los costos de transporte en vías brasileñas de bajo volumen de transito y a su vez, ampliar la investigación para determinar las interrelaciones de los principales componentes del Costo de Transporte de Vías, Costo de Construcción, Costo de Conservación y Costo de Operación de vehículos.

El objetivo principal del gobierno brasileño era minimizar los costos de transporte en vías de bajo volumen de tránsito. Esto se lograría mediante una herramienta que le permitiera evaluar en forma global el conjunto de alternativas disponibles, en los ámbitos de la construcción de nuevas vías, mejoramiento de la servicialidad de las vías existentes y operaciones de conservación de las mismas.

A partir de este estudio nace la versión tercera del Modelo de Normas de Diseño y Conservación de carreteras (Highway Design and Maintenance Satandards Model - HDM), programa destinado a evaluar económicamente las distintas alternativas de transporte en vías pavimentadas y no pavimentadas.

Con anterioridad a este estudio, Brasil no contaba con una base de datos que sirviera para el desarrollo del proyecto, por lo que inicialmente se estimó un período de 42 meses, que por diferentes razones se amplió en 24 meses para enriquecer la base de datos.

\subsubsection{Factores Relevante en el Estudio}

El estudio comprendió una serie de variables independientes, capaces de influenciar en el deterioro del camino. Esta selección se basó en la observación de diferentes tramos de vías del Brasil, así como de evaluaciones de investigaciones anteriores del tema. Estas variables independientes, factores relevantes, resultaron ser:

- Tipo de material de la capa de rodadura.

- Tránsito medio diario anual - TMDA.

- Geometría vertical. 
- Geometría horizontal.

- Frecuencia de reperfilado.

Los equipos e instrumentos utilizados para medir estos factores relevantes se detallan a continuación:

- Medición de rugosidad: Perfilómetro MAYS - RIDE - METER.

- Medición de pendiente: Gradómetro.

- Medición de curvatura horizontal: Girocompás aeronáutico direccional.

- Medición de distancia horizontal: Odómetro.

- Contador de tránsito: Automático.

- Medidor de profundidad de huellas: Triángulo de aluminio con barra graduada.

- Equipo de medición de material suelto: Flexómetro y escuadras.

- Pluviómetros: Automático y manual.

\subsubsection{Modos de Deterioro}

En vías no pavimentadas se pueden diferenciar cuatro modos de deterioro inducidos por el tránsito, estos son:

- Deterioro de superficie en la estación seca, evidenciado por el aumento de rugosidad.

- Deterioro de superficie en estación húmeda en los casos donde existe buen drenaje y los materiales de la capa de rodadura poseen suficiente capacidad de soporte (CBR) para soportar los esfuerzos producidos por las cargas del tránsito.

- Deterioro de superficie cuyo material posee baja resistencia al corte cuando está húmedo. Esto se encuentra durante la estación húmeda.

- Deformación de la capa de rodadura durante la estación húmeda. Esto 
ocurre cuando el material tiene una baja capacidad de soporte (CBR) y el espesor de la superficie es insuficiente para reducir las tensiones en la sub-base dentro de los límites de acomodo del material.

\subsubsection{Elección de Modelos de Deterioro}

En la selección de un modelo que pudiese presentar la curva de deterioro, hay dos restricciones importantes:

- Que el modelo pudiese ser transformado a una forma lineal, de modo que la cantidad de ciclos de deterioro puedan ser estudiados a través de técnicas de regresión lineal.

- Que los datos permitiesen el desarrollo del modelo.

El análisis de los datos indica que la tasa de crecimiento de rugosidad con respecto al tiempo o al tránsito, es una función del nivel de rugosidad actual $\left(\mathrm{QI}^{\star}\right)$ y no del nivel inicial de rugosidad después del reperfilado, como algunos pueden sugerir. Esto significa que:

$$
\frac{d Q I^{*}}{d t}=f\left(Q I^{*}\right)
$$

El primer modelo analizado fue la curva típica en forma de "S", Logia, cuya expresión matemática es:

$$
Q I^{*}=Q I *{ }_{\min }+\frac{\left(Q I{ }^{*} \max -Q I *{ }_{\min }\right)}{(1+E X P(-t \times f)}
$$

Donde:

$$
\begin{aligned}
& \text { QI* : Rugosidad en QI } \\
& \text { t : Tiempo en días } \\
& \text { f : Función de regresión, que es una combinación lineal de las }
\end{aligned}
$$


La mayor ventaja de este modelo es que puede ser fácilmente transformado a una forma lineal.

Otro modelo que podría representar la curva supuesta de deterioro es una combinación de dos curvas exponenciales. Debido a la escasez de datos en la gama más alta de la rugosidad, la curva que mejor se ajustaría a los datos, sería una curva exponencial con forma similar a la curva " $S$ " en la gama más baja de la rugosidad, pero que continuase creciendo a partir de ahí. La expresión matemática sería entonces:

$$
Q I^{*}=E X P(f 1+t \times f)
$$

Donde:

f1 : Parámetro de posición de inicio de la curva.

t : Tiempo en días

f : Función de regresión, que es una combinación lineal de las variables independientes.

Una ventaja de ambos modelos, el Logia y el Exponencial, es que debido a que la desviación estándar está relacionada con la magnitud de la rugosidad, una transformación logarítmica produce una varianza homogénea para el análisis de regresión. El modelo exponencial fue preferido por ser más fácil de trabajar en computación.

\subsubsection{Modelo de Variación de Rugosidad en el Tiempo}

Para la función exponencial Rugosidad - Tiempo se utilizo el conjunto de Paquete de Programas Estadísticos Statistical Análisis System (SAS), obteniéndose el siguiente modelo:

$$
\begin{aligned}
L D Q= & D(0,4314-0,1705 T 2+0,001159 N A+0,000895 N C-0,000227 N C * R \\
& +E(-0,1442-0,0198 R+0,00621 P N-0,01421 P-0,000617 N A))
\end{aligned}
$$

Donde: 
LDQ : Variación del logaritmo natural de rugosidad (QI)

D : Número de días desde el último reperfilado, en centenas (tiempo/100).

T2 : Variable indicadora del tipo de material de carpeta de rodado:

$\mathrm{T} 2=1$, si es tierra.

$\mathrm{T} 2=0$, si es otro.

NA : Tránsito medio diario de automóviles y utilitarios, en ambas direcciones.

NC : Tránsito medio diario de buses y camiones, en ambas direcciones.

$\mathrm{R}$ : Valor absoluto de la pendiente longitudinal media, en porcentaje.

E : Variable indicadora de estación:

$E=0$, si es estación seca

$E=1$, si es estación lluviosa

PN : Porcentaje de material de la capa de rodadura que pasa por el tamiz 0,074 mm (malla 200).

IP : Índice de plasticidad del material de la capa de rodadura (\%)

Este modelo tiene un valor de $\mathrm{R}^{2}$ igual a 0,26 , con un tamaño de muestra de 8.276 observaciones.

Se observa que el valor de $\mathrm{R}^{2}$ es bajo, debido a la gran variabilidad de la rugosidad en la dirección transversal y longitudinal de los tramos estudiados. Sin embargo, como el número de observaciones es muy grande, la ecuación es estadísticamente significativa.

Para mejorar el modelo anterior se consideró como variable la precipitación acumulada desde el último reperfilado (PPA en $\mathrm{mm}$ ), resultando: 
$L Q D=D(0,3759-0,101 T 2+0,0032 N A+0,00101 N C)+P P A(-0,00016-$ $0,0000354 R+0,00000883 P N-0,0000218 I P))$

Este modelo tiene un valor de $\mathrm{R}^{2}$ igual a 0,31 , con un tamaño de muestra igual al anterior, por lo que también es estadísticamente significativa.

También se evaluó el modelo de rugosidad después del reperfilado, concluyéndose que en este caso, ella depende principalmente de la habilidad del operador de la motoniveladora. Se observa que los puntos medidos no siguen un patrón y varían de posición en forma aleatoria con el tiempo, corroborando la declaración de que la correlación entre Rugosidad y Tiempo es baja debido a que cambia la posición de las huellas de las ruedas con el correr del tiempo.

También se puede concluir que las propiedades de los materiales de la capa de rodadura, no explican satisfactoriamente su influencia en la predicción de la variación de rugosidad con el tiempo.

\subsubsection{Modelo de Pérdida de Grava en el Tiempo}

La perdida de grava está definida como la variación de espesor de grava a lo largo de un periodo de tiempo. Los enripiados dan inicio a un nuevo ciclo de análisis de perdida de grava. Si no hubiese ripio, el intervalo de análisis abarcaría desde la primera observación hasta el reperfilado siguiente.

Los tres factores identificados como los que más influyen en la pérdida de material son: La acción del clima, el tránsito y la conservación representada por el reperfilado.

El modelo quedó representado en la siguiente expresión matemática:

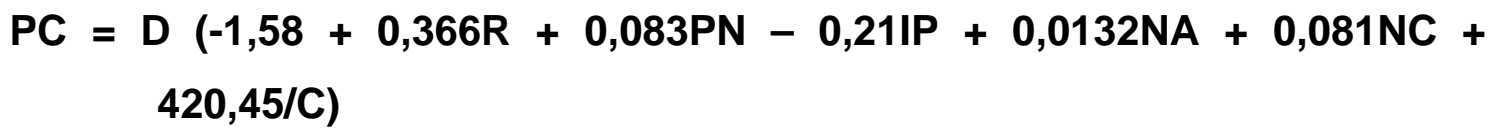
$420,45 / C)$

Donde:

PC : Pérdida de espesor de grava en milímetros 
D : Intervalo de tiempo considerado en centenas de días (tiempo/100).

$\mathrm{R} \quad$ : Valor absoluto de la pendiente en porcentaje.

IP : Índice de plasticidad en porcentaje.

PN : Porcentaje de material de la capa de rodadura que pasa por el tamiz 0,074 mm (malla 200).

NA : Tránsito medio diario de automóviles y utilitarios en ambas direcciones.

NC : Tránsito medio diario de buses y camiones en ambas direcciones.

C : Radio de curvatura horizontal en metros.

El valor de $R^{2}$ de este modelo es igual a 0,60 y el tamaño de muestra utilizada fue de 604 observaciones.

En función de la ecuación anteriormente descrita se puede observar lo siguiente:

- La perdida de grava aumenta con la pendiente, el material fino, el tránsito de los vehículos, livianos y pesados, y cuando disminuye el radio de curvatura.

- Cuando el índice de plasticidad aumenta, la perdida de grava disminuye, ya que el índice representa la capacidad de aglutinamiento de material fino.

- A medida que aumenta la frecuencia de reperfilado disminuye la pérdida de grava.

Se realizó sin éxito la tentativa de incluir en el modelo la precipitación pluviométrica acumulada, ya que los resultados fueron contrarios a la experiencia. 


\subsubsection{Conclusiones del Estudio Brasileño}

Los investigadores concluyeron que los resultados obtenidos eran inadecuados para uso generalizado, motivo por el cual deberían ser usados con cautela y reserva. Se recomendaron estudios adicionales para obtener modelos más realistas y confiables que puedan ser ampliamente utilizados en Planeamiento Vial.

Posteriormente, entre los años 1983 y 1985, Williams Paterson, investigador del Banco Mundial, realizó un análisis para suprimir ciertas tendencias altas del modelo y estimó la rugosidad promedio durante el ciclo de reperfilado, expresiones de rugosidad mínima, máxima y variación de la rugosidad en el tiempo. Estas expresiones están incorporadas en los modelos del HDM-III.

\subsection{VALIDACIÓN DEL MODELO DE DETERIORO - LORENA ARAYA ${ }^{11}$}

El objetivo del experimento para la validación de los modelos de deterioro realizados por Lorena Araya, fue lograr una toma de datos en terreno para realizar el análisis del modelo de deterioro de caminos no pavimentados. En este estudio las características analizadas fueron geometría, tránsito, propiedades del suelo y la rugosidad que presentaban los caminos en el periodo de estudio.

La tabla siguiente nos detalla un resumen de las características de los caminos considerados en el estudio. La toma de datos se realizó entre los años 1986 y 1987.

Los caminos no pavimentados seleccionados para este estudio fueron un total de siete, eligiendo de entre ellos 15 tramos. Para cuantificar el deterioro de los caminos seleccionados, el estudio se abocó en forma especial a medir la evolución de la rugosidad durante un período de tiempo, considerando éste como el factor más representativo del deterioro para esta clase de caminos. El período de observación abarcó alrededor de un año e incluyo una estación

\footnotetext{
${ }^{11}$ Araya Carvajal, Lorena (1988). Validación del Modelo de Deterioro Brasileño de Caminos no Pavimentados. Aplicación al caso chileno. Memoria para optar al título de Ingeniero Civil, Universidad de Chile. Santiago de Chile.
} 
seca y otra húmeda.

Tabla 2.1: Características de los tramos testigos

\begin{tabular}{|c|c|c|c|c|}
\hline Variables & Media & Desv. St. & Mín & Máx \\
\hline \multicolumn{5}{|l|}{ Número de tramos: 15} \\
\hline Largo (m) & 1.343 & & 732 & 2,53 \\
\hline Pendiente (\%) & 1,9 & & 0 & 6,8 \\
\hline Ancho de la vía (m) & 7,9 & & 6 & 10 \\
\hline Curvatura (o/km) & 45 & 80 & 0 & 260 \\
\hline Precipitación (mm/año) & 656 & 290 & 179 & 1.114 \\
\hline \multicolumn{5}{|l|}{ Característica del material } \\
\hline \% Pasa malla 10 & 50 & 13 & 35 & 79 \\
\hline \% Pasa malla 40 & 34 & 10 & 21 & 52 \\
\hline \% Pasa malla 200 & 15 & 5 & 9 & 23 \\
\hline Índice Plasticidad & 3,4 & 4,8 & 0 & 12 \\
\hline Límite líquido & 7,6 & 9,6 & 0 & 25 \\
\hline \multicolumn{5}{|c|}{ Tránsito medio diario (ambos sentidos) } \\
\hline Automóviles & 108 & 36 & 42 & 147 \\
\hline Camionetas & 105 & 40 & 40 & 173 \\
\hline Camiones 2 ejes & 57 & 17 & 27 & 83 \\
\hline Camiones +2 ejes & 7 & 4 & 2 & 14 \\
\hline Buses & 10 & 6 & 25 & 4 \\
\hline \multicolumn{5}{|c|}{ Información sobre medidas de rugosidad } \\
\hline Rugosidad NAASRA (mm/km) & 11.435 & 2.947 & 4.798 & 19.337 \\
\hline Rugosidad - IRI & 13,5 & & 6,6 & 19 \\
\hline Número de Observaciones & 752 & & & \\
\hline
\end{tabular}

De los resultados obtenidos en el estudio, se destacan algunos comentarios importantes sobre el uso de los Modelos de Deterioros.

\subsubsection{Uso del Modelo de Deterioro HDM-II}

Por los resultados obtenidos en el estudio se puede afirmar que el modelo siempre estima valores por debajo de las rugosidades medidas. Por lo tanto, no 
se estaría confiando en sus resultados especialmente en la estimación de la rugosidad promedio para una cierta política de conservación.

Referente al rango de rugosidad, los datos de terreno se encuentran dentro de lo observado en el estudio de Kenya, no así las condiciones de los tramos como la plasticidad, tamaño máximo y otros, por lo cual creemos que este es el factor principal por lo que el modelo presenta un aumento de rugosidad no muy pronunciado en caminos de grava. Se recomendó medir la rugosidad mínima para ser utilizada en la ecuación de proyección de la rugosidad, con esto se estaría disminuyendo la diferencia entre lo estimado y lo real.

\subsubsection{Uso del Modelo de Deterioro HDM-III}

Los resultados que muestran los cuadros comparativos entre los datos de terreno y los valores estimados por el modelo, se encuentran dentro de los rangos aceptables, excepto el de rugosidad máxima. Para el cálculo de la rugosidad promedio, el valor de la rugosidad máxima es influyente en el resultado, por lo que conviene utilizar datos más apropiados a la realidad nacional.

\subsubsection{Conclusiones - Estudio Lorena Araya}

Finalmente el estudio de Lorena Araya concluye para los modelos del HDM-II y HDM-III lo siguiente:

\section{i) Modelo de Kenya: HDM-II}

\section{Ventajas del Modelo:}

- Fácil uso del modelo, el usuario solamente necesita el tipo de carpeta, el tránsito y la cantidad de lluvia anual.

- Los rangos de las variables chilenas están dentro de los rangos de las estudiadas en Kenya.

\section{Desventajas del Modelo:}

- Este modelo siempre se mantuvo por debajo de los datos 
medidos, lo cual puede falsear los beneficios de la conservación de caminos.

- No es generalizado. Las características del suelo (índice de plasticidad y granulometría) no se utilizan como variables de entrada en las ecuaciones. Tampoco las características geométricas, salvo en los modelos de grado de soltura del material. Se le da mucha importancia al tránsito.

- En épocas de lluvia el aumento de la rugosidad es mayor que en épocas secas. Esto se opone a lo medido en el área de estudio.

\section{Recomendaciones:}

- Para utilizar la ecuación de progresión de la rugosidad se debe conocer la rugosidad mínima. El estudio recomienda un valor de $4.400 \mathrm{~mm} / \mathrm{km} \mathrm{BI}$.

- $\quad$ Se recomienda la siguiente relación para llevar de BI a IRI:

$$
I R I=0,0032 * B I^{0,89}
$$

\section{ii) Modelo de Brasil: HDM-III}

\section{Ventajas del Modelo:}

- Es más generalizado. Considera características del suelo, geometría condiciones ambientales y tipo de vehículo.

- En épocas de lluvia resultó ser un buen modelo de simulación de rugosidad.

- El rango de rugosidad es más amplio que el de Kenya, lo mismo que el tránsito.

- La estimación de la rugosidad mínima estuvo dentro del rango medido en el área de estudio. 


\section{Desventajas del Modelo:}

- El modelo sobrestima la rugosidad cuando la política de conservación considera bajas frecuencias de reperfilados, menores a tres reperfilados anuales.

- El modelo subestima la rugosidad cuando la política de conservación considera altas frecuencias de reperfilado, mayores a ocho reperfilados anuales.

- La estimación de la rugosidad máxima para los tramos fue muy alta comparada con la medida. Por otra parte, esto corrige la tendencia a subestimar que poseía el modelo de Kenya.

- El modelo brasileño fue diseñado para zonas lluviosas, por lo que pierde su validez en regiones secas. Se debe tener en cuenta que la lluvia es una variable importante en el modelo de deterioro.

- El modelo de deterioro brasileño presenta una baja correlación, pero fue aceptado estadísticamente por la gran cantidad de datos tomados.

\section{Recomendaciones:}

- Al igual que el modelo de Kenya, se recomienda no dejar que el modelo estime las rugosidades de entrada, como son la mínima y máxima. En este estudio se llegó a valores aproximados de 85 QI y 159 QI, es decir, 6,5 IRI y 12,2 IRI respectivamente.

- Se debe tener en cuenta que al realizar una evaluación económica, el modelo escoge siempre las políticas de conservación que tiene mayor frecuencia de reperfilado.

Respecto de la investigación y los datos tomados en el área de estudio se concluyó que:

- El deterioro de los caminos no pavimentados en épocas secas es 
mayor que en estaciones lluviosas. Esto puede deberse al bajo porcentaje de material fino cohesivo en las estructuras granulares de la región central, y a valores bajo de humedad de las capas granulares, reduciendo su capacidad resistente.

- La variación de la rugosidad producto de un reperfilado es del orden de un 25\% (3.000 a 4.000 mm/km NAASRA).

- A medida que la carpeta de rodadura presenta mayor tamaño máximo, mayor es el promedio de la rugosidad en el tramo.

- Las características geométricas no reflejaron gran influencia en el deterioro de los caminos. Sin embargo, en épocas lluviosas, los caminos con pendiente y curvatura presentaron una menor rugosidad promedio. Este fenómeno puede deberse a una mejora de la drenabilidad de la superficie de la capa granular debido a la pendiente longitudinal y transversal (curvas), y en razón a una mayor porosidad por déficit de finos en la estructura granular.

\subsection{COMPORTAMIENTO CAMINOS NO PAVIMENTADOS - JOSE GARCÍA ${ }^{12}$}

Los modelos de deterioro que se aplican en Chile se basan en el análisis del estudio de Brasil - PNUD ${ }^{13}$. Como se expresó en el punto anterior, dichos modelos fueron validados en el estudio de Lorena Araya ${ }^{14}$, en el cual se cuestiona su uso directo. El trabajo efectuado por José García tuvo como objetivo calibrar los modelos de deterioro ocupados en la actualidad y además proponer políticas de conservación, con frecuencias y/o umbrales predefinidos que respondan a un nivel de exigencias basándose en lo obtenido en la práctica y los resultados a través del modelo HDM-III.

Para lograr la calibración de los modelos de deterioro del HDM-III, se efectúo

\footnotetext{
${ }^{12}$ García Aranzaes, José (1993). Estudios de caminos no pavimentados de la zona central de Chile. Memoria para optar al título de Ingeniero Civil. Universidad de Chile. Santiago de Chile. ${ }^{13}$ William Paterson D.O. (1987). "Road Deterioration and Maintenance Effects: Models for Planning and Management. Highway Desing and Maintenance Standards Series", World Bank Transportation department, Washington D.C.

${ }^{14}$ Araya Carvajal, Lorena (1988). Pavimentados. Aplicación al caso chileno. Memoria para optar al título de Ingeniero Civil, Universidad de Chile. Santiago de Chile
} 
un seguimiento de las variables de Rugosidad y Pérdida de Material en diferentes tramos de algunos caminos de la zona central de Chile.

Las mediciones de rugosidad abarcaron el período comprendido entre noviembre del año 1992 y agosto de 1993.

\subsubsection{Resultados de la Medición de la Pérdida de Material}

Para medir la Pérdida de Material se utilizaron dos métodos de mediciones, uno de ellos fue el método de las calicatas, que no es otra cosa que sondeos que permiten observar y medir el espesor de carpeta remanente. El otro método utilizado fue el topográfico, que mide directamente la disminución del espesor de la carpeta efectuando una nivelación con respecto a una cota conocida.

Se puede ver que los resultados en promedio arrojados dicen que la Pérdida de Material alcanza aproximadamente a unos $40 \mathrm{~mm}$ al año, esto medido con el método de las calicatas que presenta una gran dispersión al observar las mediciones particulares. Por otra parte, el método topográfico obtuvo mediciones más estables que arrojaron valores mayores, del orden de $64 \mathrm{~mm}$ al año.

\subsubsection{Análisis de Rugosidad en los Tramos Testigos}

\section{i) Rugosidades Mínimas}

El modelo del efecto de Reperfilado, nace de la condición de rugosidad más baja que puede alcanzar el camino ( $\left.R I_{\text {mín}}\right)$ y sobre la cual las actividades de mantenimiento no tienen mayor efecto.

La rugosidad mínima $\left(R I_{\text {mín}}\right)$, es la aspereza más baja que puede alcanzar un camino, bajo la cual no se puede reducir más el grado de ésta. La rugosidad mínima depende del tamaño máximo de las partículas del material y de la pendiente de gradación media del material.

Del conjunto de datos tomados en terreno, la rugosidad mínima alcanzada fue de $4.676 \mathrm{~mm} / \mathrm{km}$ ó $6,4 \mathrm{~m} / \mathrm{km}$ IRI. Además, no se apreció 
una relación directa entre la rugosidad mínima y el tamaño máximo de la partícula o el tránsito medio diario anual (TMDA).

\section{ii) Rugosidad Máxima}

El modelo adoptado por el HDM circunscribe a la rugosidad o aspereza, a un límite superior alto, rugosidad máxima $\left(R I_{\max }\right)$, desde la cual una función convexa decreciente con tasa exponencial inversa desciende hasta el valor esperado. La rugosidad máxima es función de las propiedades de los materiales y la geometría del camino.

Se puede apreciar de los datos del estudio, que la rugosidad máxima alcanzada en las mediciones fue de 18.578 mm/km ó 18,6 m/km IRI.

\section{iii) Análisis de Rugosidades Antes y Después del Reperfilado}

Basados en los gráficos de rugosidad en función del tiempo de algunos caminos estudiados, se pudo extrapolar las rugosidades aproximadas antes y después del reperfilado. El resultado obtenido indica que existe una variación del 46\% entre la rugosidad del antes y del después del reperfilado.

\subsubsection{Rugosidad en las Estaciones Climáticas}

En el estudio se pudo apreciar claramente que a pesar del corto tiempo de lluvia analizado, se presentó una rugosidad promedio mayor en prácticamente todos los tramos, en comparación con el período seco.

A pesar que los resultados del modelo contradicen lo obtenido en el estudio chileno para la misma zona, se considera que representa efectivamente la evolución de la rugosidad, ya que de acuerdo a lo observado en el período de lluvias, el agua al acumularse sobre la superficie de la carpeta sumado al paso de los vehículos, los baches se producen con mayor facilidad, es decir, al existir un mal bombeo del camino debido a la acumulación de material a los costados del camino, estos evitan que el agua drene rápidamente de la superficie de la carpeta de rodado; esto sumado a un cierto tránsito de 
vehículos, se produce un aumento de los baches, las grietas de erosión por efecto drenante de las aguas lluvias y por ende la rugosidad.

Este resultado concuerda con lo obtenido en estudios de otros países en donde la rugosidad aumenta al llegar el período de lluvias, es por ello que a pesar de todos los errores asociados en este tipo de mediciones, se consideró que los resultados efectivamente muestran la evolución de la rugosidad.

\subsubsection{Calibración de los Modelos de Deterioro}

La intención de efectuar la calibración, no fue otra cosa que una forma de ajustar los modelos originales desarrollados por Paterson en su estudio de Brasil, conservando sus formas funcionales y modificar los coeficientes para acomodar los resultados a la realidad chilena.

\section{i) Calibración del Modelo de Rugosidad}

El modelo de rugosidad mostrado en su forma analítica en el estudio brasileño se sustenta entre otros aspectos en un submodelo de rugosidad máxima, por lo que se partió por calibrar esta fórmula inicial. El resultado obtenido en la calibración fue el siguiente:

$$
\mathrm{QI}_{\max }=156,9958+661,9011 *(0,5-\mathrm{MGD})^{2}-0,63102 * \mathrm{RF}^{*} \mathrm{MMP}-4.804,65 * \mathrm{KCV}
$$

Donde:

$$
\begin{aligned}
& \mathrm{KCV}=\quad \text { Curvatura horizontal promedio del camino, en grados } / \mathrm{km} \\
& \mathrm{RF}=\text { Promedio de subidas más bajadas del camino, en } \mathrm{m} / \mathrm{km} \\
& \mathrm{MGD}=\quad \text { Relación del material en gradación polvo } \\
& \mathrm{MMP}=\quad \text { Precipitación media mensual, en } \mathrm{mm} / \mathrm{mes}
\end{aligned}
$$

Para esta calibración se llegó a un error estándar de 23,70 y un coeficiente de correlación múltiple de $\mathrm{R}^{2}=0,35$. Esta baja correlación es mayor que la obtenida por los modelos brasileños, considerando este modelo más ajustado al caso chileno. 
Para calibrar el modelo completo de progresión de la rugosidad, se procedió a obtener el parámetro "c" encontrado por Paterson ${ }^{15}$ y que está determinada por la relación:

$$
\mathrm{QI}_{\mathrm{TG2}}=\mathrm{QI}_{\max }-\mathbf{b}{ }^{*}\left(\mathrm{Q} \mathrm{I}_{\max }-\mathrm{QI}_{\mathrm{TG} 1}\right)
$$

Donde:

$$
\mathrm{b}=\exp \left[\mathrm{c} *\left(\mathrm{TG}_{2}-\mathrm{TG}_{1}\right)\right] \quad \text { donde: } 0<\mathrm{b}<1
$$$$
c=-0,001 *\left[0,461+0,0174^{\star}(\mathrm{ADL})+0,0114^{\star}(\mathrm{ADH})-0,0287^{\star}(\mathrm{ADT})^{\star}(\mathrm{MMP} / 1000)\right]
$$

En tanto que las variables son:

$\mathrm{RI}_{\mathrm{TG} 1}=$ Rugosidad al tiempo $\mathrm{TG}_{1}$, en $\mathrm{m} / \mathrm{km} \mathrm{IRI}$

$\mathrm{RI}_{\mathrm{TG} 2}=$ Rugosidad al tiempo $\mathrm{TG}_{2}$, en $\mathrm{m} / \mathrm{km} \mathrm{IRI}$

$\mathrm{TG}_{\mathrm{i}}=$ Tiempo desde la última nivelación, en días

$\mathrm{ADL}=$ Tránsito medio diario liviano (bajo $3.500 \mathrm{Kg}$ ) en ambas direcciones, en vehículos/días

$\mathrm{ADH}=$ Tránsito medio diario pesado (sobre $3.500 \mathrm{Kg}$ ) en ambas direcciones, en vehículos/días

$\mathrm{ADT}=$ Todo el tránsito medio diario (livianos más pesados) en ambas direcciones, en vehículos/días

El ajuste del parámetro "c" entregó el siguiente resultado:

$$
c=0,451941-0,00261 \text { * ADL + 0,005967 * ADH }-0,07158296 \text { * ADT * MMP }
$$

Con un error estándar de 0,25 y un coeficiente de correlación $R^{2}=0,040$, mostrando una baja correlación del modelo con lo medido en el estudio. Dado los resultados obtenidos en este modelo de progresión de rugosidad, se decidió solo linealizar la rugosidad máxima.

15 William Paterson D.O. (1987). "Road Deterioration and Maintenance Effects: Models for Planning and Management. Highway Desing and Maintenance Standards Series", World Bank Transportation department, Washington D.C. 
Posteriormente como una forma de verificar el grado de incidencia de los factores más importantes que influyen en estos modelos y comprobar la variabilidad que presentan, se realizó un análisis de sensibilidad.

Los resultados demostraron que efectivamente la progresión propuesta ajusta la predicción de la rugosidad máxima a lo observado, pero se consideró que no es suficientemente sensible a factores importantes como lo son el tránsito y la lluvia en los porcentajes señalados. De este modo, su uso no es recomendable.

Se propuso como alternativa ingresar la rugosidad máxima ( $\mathrm{QI}$ max $)_{\text {con }}$ un valor de 273 QI ó 21 IRI como dato de entrada del modelo, valor que fue el máximo obtenido en el estudio.

\section{ii) Calibración del Modelo de Reperfilado}

La ecuación para estimar la rugosidad después del reperfilado, se expresa como función lineal de la rugosidad antes del reperfilado, de la pendiente de gradación media del material (MG) y la rugosidad mínima.

El resultado de la calibración fue:

$$
Q I_{\min }=-19,906 \text { * D95 - 0,07753 * (D95 * MG) + 119,26552 }
$$

Con un error estándar igual a 25.3 Ql y un coeficiente de correlación $\mathrm{R}^{2}$ = 0,010, demostrando que dada esta ínfima correlación el modelo no es aplicable al caso chileno. Se propone como alternativa ingresar la rugosidad mínima como dato de entrada al ejecutar el programa, ocupando los siguientes valores:

$$
\begin{aligned}
& \mathrm{QI}_{\min }=83 \text { QI para caminos de grava } \\
& \mathrm{QI}_{\min }=90 \text { QI para caminos de tierra }
\end{aligned}
$$

Con la salvedad anterior se procedió a calibrar la relación que da cuenta del efecto de reperfilado. Para ello se intentó obtener el parámetro "a" de la expresión: 


$$
Q I_{\text {después }}=Q I_{\min }+a^{*}\left(Q I_{\text {antes }}-Q I_{\text {min }}\right)
$$

El resultado de esta operación fue:

$$
a=-0,2617+1,38738 * M G D
$$

La regresión entregó un error estándar de 0,114 y un coeficiente de correlación $R^{2}=0,629$.

Se debe señalar además, que el efecto de reperfilado baja la rugosidad en un $45 \%$ aproximadamente, entre la situación antes de reperfilar y la condición final.

Al comparar los datos medidos con el propuesto y el original, se concluyó que el orden de magnitud de los resultados de ambos es muy similar, teniendo el modelo propuesto una mejor aproximación a los valores medidos.

Se recomendó ajustar este efecto introduciendo la rugosidad mínima como dato de entrada en el programa (HDM - III) e incorporando los valores encontrados para el factor "a".

Los restantes modelos que involucra la rugosidad no necesitan ajustes al caso chileno, ya que corresponden a un análisis matemático de la rugosidad media en el año y de reperfiladura frecuente que se basan en los modelos ya analizados.

En cuanto al modelo del efecto de la compactación sobre la progresión de la rugosidad, no fue posible analizarlo, ya que considera recebos con y sin compactación mecánica, que por razones de tiempo y recursos no resultaron posibles de realizar en el estudio.

\section{iii) Calibración del Modelo de Pérdida de Material}

El segundo modelo de deterioro del HDM - III, está referido a la cuantificación de la Pérdida de Material, es decir, intenta predecir la disminución del espesor de la carpeta de grava o tierra del camino. 
Con los pocos datos que se tenían para efectuar la calibración se llegó al siguiente resultado:

\section{$M L A=72,346+76,998 * M M P * R F+0,0661$ * ADT $-2.095,0$ * ADT * MMP * PI - 0,34278 * ADT*MMP * P075 - 12,2067 * MMP * ADT}

MLA = Pérdida anual de material, en mm/año

MMP = Precipitación media mensual $(\mathrm{mm} / \mathrm{mes})$

P075 = Porcentaje de material que pasa malla \# 200

$\mathrm{PI} \quad=$ Índice de plasticidad

$\mathrm{RF} \quad=$ Subidas más bajadas $(\mathrm{m} / \mathrm{km})$

ADT = Tránsito medio diario anual (vehículos/día)

El error estándar obtenido del análisis fue de $3 \mathrm{~mm}$ y un coeficiente de correlación $\mathrm{R}^{2}=0,999$, valor que indicaría una gran precisión entre el modelo y los resultados empíricos, pero como se trata de un número mínimo de mediciones (9), con respecto al número de variables, no corresponde a un buen indicador en este caso.

Al realizar un análisis de sensibilidad del modelo, se comprobó que al igual que el modelo original, falla al aumentar la precipitación promedio mensual y el tránsito, prediciendo una menor pérdida de material para estos casos. Esto indicaría que el modelo no debe aplicarse en nuestro país, por lo que es necesario realizar un estudio más acabado para tener un modelo definitivo.

\subsubsection{Conclusión estudio José García}

El estudio tuvo como objetivo último, ajustar los modelos de deterioro para caminos no pavimentados que incluye el HDM - III y señalar las tendencias en el deterioro de estos caminos.

Se comprobó que los modelos no representan fielmente el caso chileno, ya que 
poseen muy baja correlación con los datos medidos, por lo que estos no pudieron ser ajustados. Además se pudo detectar que el modelo de Pérdida de Material contradice lo que sucede en la realidad para los rangos de material fino que se utiliza en nuestro país.

Se considera que los coeficientes de correlación encontrados al analizar el comportamiento de rugosidad en el tiempo, no pueden ser aceptados como razonables, a pesar del gran error asociado a este tipo de mediciones, ya que se trata de un número muy pequeño de datos. Se estima que las diferencias detectadas se deben principalmente a dos razones:

- Al rango de precipitación media mensual, que en el caso brasileño supera aproximadamente 4 veces el de la zona central del país; y

- Al porcentajes de fino de los materiales constitutivos de los caminos chilenos respectos de los brasileños, fuente de los modelos incorporados en el HDM - III.

\section{i) Tendencias Encontradas en el Estudio}

- El rango de rugosidad media para caminos no pavimentados de la zona de estudio fluctúa entre 6.3 y $21 \mathrm{~m} / \mathrm{km}$ IRI. De acuerdo a lo detectado, se puede alcanzar valores de hasta $25 \mathrm{~m} / \mathrm{km}$ IRI en tramos muy cortos (aproximadamente 50 metros).

- La perdida de material en la zona de estudio varía entre 0 y $13 \mathrm{~cm}$ anuales de acuerdo a lo medido. Estos valores son cuestionables ya que la forma en que fueron determinados no fue con la precisión y exactitud requerida, debido a la carencia de medios para realizar mediciones con menor error asociado, se sugirió en un futuro estudio implementar nuevos sistemas de medición.

- De acuerdo a lo medido se encontró que la geometría no es un factor muy determinante en el deterioro de caminos, salvo en periodos de lluvias en que caminos con gran pendiente (> 5\%) se conservan mejor que los de pendiente baja. La explicación de acuerdo a lo 
observado puede deberse a que en épocas de lluvia disminuye considerablemente el tránsito y la pendiente mejora la drenabilidad, evacuando en menor tiempo el agua de la superficie del camino. En tanto que en caminos con baja pendiente, estos son mal drenados, acumulándose con mayor facilidad el agua sobre la capa superficial; sumado el efecto del tránsito, nos encontramos con que se produce, con gran rapidez, una gran cantidad de baches de tamaños variables, con el consiguiente aumento de la rugosidad.

- Se comprobó que existe una relación muy estrecha entre la rugosidad y la conservación aplicada.

- Se encontró que el efecto del reperfilado disminuye la rugosidad del camino en un $45 \%$ como promedio, siendo los valores extremos de $20 \%$ y $60 \%$ como mínima y máxima respectivamente.

- Se comprobó que la operación de conservación de reperfilado con compactación mecánica y riego, disminuye la rugosidad en $65 \%$ lo que equivale a $8 \mathrm{~m} / \mathrm{km} \mathrm{IRI}$, pero su efecto sobre el camino no perdura más de 45 días y su costo es muy superior al de un reperfilado simple.

- Se detectó que las operaciones de conservación de recebo y bacheo no son tan malas como el modelo predice y de acuerdo a lo observado en este estudio se recomienda ejecutarlas acompañadas por un reperfilado 3 a 4 veces durante el año.

- Se encontró que el efecto del bacheo baja la rugosidad en un $39 \%$ como promedio, lo que equivale a $6 \mathrm{~m} / \mathrm{km} \mathrm{IRI} \mathrm{aproximadamente,} \mathrm{este}$ valor se determinó a través de las mediciones hechas en un solo tramo, y corresponde al caso particular ya que no pudo ser implementada esta operación en otros caminos.

- No se detectaron relaciones entre las propiedades geotécnicas de la carpeta de rodado del camino y las variables estudiadas, esto se 
debió principalmente a la aplicación de distintas políticas de conservación en cada caso.

\section{ii) Ajuste de los Modelos de Deterioro del HDM - III}

A pesar que los modelos no representan fielmente el caso chileno, se logró encontrar rangos que limitan las tendencias de éstos. En el caso de la rugosidad máxima se encontraron dos valores:

- $Q I_{\max }(1)=242$ QI: Este valor corresponde al máximo medido en el seguimiento de la evolución de la rugosidad. Es más representativo para caminos que tengan al menos una conservación al año. Se observó que los caminos sin conservación presentaban rugosidades cuyo crecimiento disminuía al superar los 17000 mm/km o 17 m/km IRI, es decir, la pendiente de la curva en este caso tendía a cero.

- $Q I_{\max }(2)=273$ QI: Este valor corresponde al máximo medido en el estudio y cuya forma de medición de rugosidad difiere del método tradicional y corresponde a un camino en pésimas condiciones, con sobretamaño y muy bacheado lo que no representa el promedio de los caminos no pavimentados de nuestro país.

En el caso de la rugosidad mínima ( $\left(\mathrm{QI}_{\min }\right)$ se encontró el valor de 6.4 m/km ó 4676 mm/km Naasra, correspondiendo al efecto producido por un reperfilado con compactación y riego quedando el camino en óptimas condiciones.

Se recomienda introducir estos valores extremos $\mathrm{QI}_{\max }=21 \mathrm{~m} / \mathrm{km} \mathrm{IRI} \mathrm{y}$ $\mathrm{QI}_{\min }=6.4 \mathrm{~m} / \mathrm{km} \mathrm{IRI}$ como datos de ingreso del HDM-III, evitando las predicciones erradas del modelo.

El efecto del reperfilado puede ser ajustado a los valores encontrados en este estudio. Con respecto a la pérdida de material se propone hacer un estudio más extenso y minucioso para determinar un nuevo modelo que se ajuste al caso chileno. 


\section{CAPITULO III}

\section{ESTUDIO DE CAMINOS NO PAVIMENTADOS ESTABILIZADOS CON BISCHOFITA EN LA REGIÓN DE ANTOFAGASTA - CHILE}

\subsection{INTRODUCCION}

Asumiendo que la aplicación directa de las expresiones encontradas para el caso brasileño, no representan fielmente lo que acontece en la realidad chilena y sus regiones, por ser un territorio muy disímil al brasileño, además de presentar en toda su extensión diferentes zonas climáticas, tipos de suelos, topografía, tránsito, etc., es que se plantea la necesidad de ajustar el modelo HDM-4 a la realidad chilena, en particular a la Región de Antofagasta.

Para llevar a cabo el ajuste de los modelos de deterioro que cuenta el HDM-IV, fue necesario hacer un seguimiento a las variables de Rugosidad y Pérdida de Material en diferentes tramos de algunos caminos de la Región de Antofagasta de Chile.

El desarrollo de este estudio no podría haber sido posible sin el apoyo y colaboración de la Dirección de Vialidad del MOP de la II Región, en especial del Departamento de Proyectos de Vialidad de Antofagasta, el Laboratorio Nacional de Vialidad (LNV) y del Laboratorio Regional de Vialidad.

\subsection{OBJETIVO DEL ESTUDIO}

La importancia del proceso de ajustar los modelos de comportamiento está en el impacto económico, ya que debido a la influencia directa en el inicio y progresión de los deterioros del camino, se hace necesaria una adecuada adaptación de ellos, de modo que se pueda efectuar una evaluación económica certera de los proyectos viales.

En el caso de las capas granulares, los modelos de deterioro consideran dos variables de respuesta; la rugosidad que es un indicador y que afecta los costos de operación de los vehículos y la perdida de material que es el principal deterioro asociado a los costos de conservación. 
A su vez, una correcta evaluación económica nos permitirá definir una eficiente planificación en el contexto del ciclo de gestión. Recordemos que la planificación comprende el análisis del sistema de carreteras en su conjunto y, típicamente, requiere la preparación de presupuestos a medio y largo plazo o estratégicos, de estimaciones de gastos de desarrollo y conservación de carreteras bajo diferentes supuestos económicos y presupuestarios. Se pueden hacer previsiones de las condiciones de redes de carreteras bajo diversos niveles de financiación en términos de indicadores clave, junto con previsiones de los gastos necesarios bajo partidas presupuestarias definidas.

Es por ello que el objetivo principal de la investigación es ajustar los modelos de comportamiento de caminos no pavimentados del HDM-4, específicamente los modelos de rugosidad y pérdida de material, para las vías no pavimentadas de la Región de Antofagasta de Chile, según las características propias de las mismas.,

Con respecto a la progresión de la rugosidad, el modelo existente predice esta variable con valores que escapan de los resultados empíricos existentes, siendo necesario efectuar un ajuste de los actuales modelos. Para tal efecto, se utilizarán mediciones efectuadas en terreno y existentes de rugosidad y de pérdida de material; las consideraciones y recomendaciones efectuadas a los modelos brasileños expuestos en los estudios hasta hoy realizados, y las actividades de conservación de la Dirección Regional de Vialidad de Chile.

\section{3 ÁREA DE ESTUDIO}

El área de estudio donde se realizó el seguimiento de los caminos no pavimentados de red vial chilena, fue la zona correspondiente a la Región de Antofagasta. El trabajo se limitó solo a los caminos no pavimentados estabilizados con bischofita de esta zona, debido a restricciones de recursos y disponibilidad del equipo de rugosidad del Laboratorio Nacional de Vialidad. 

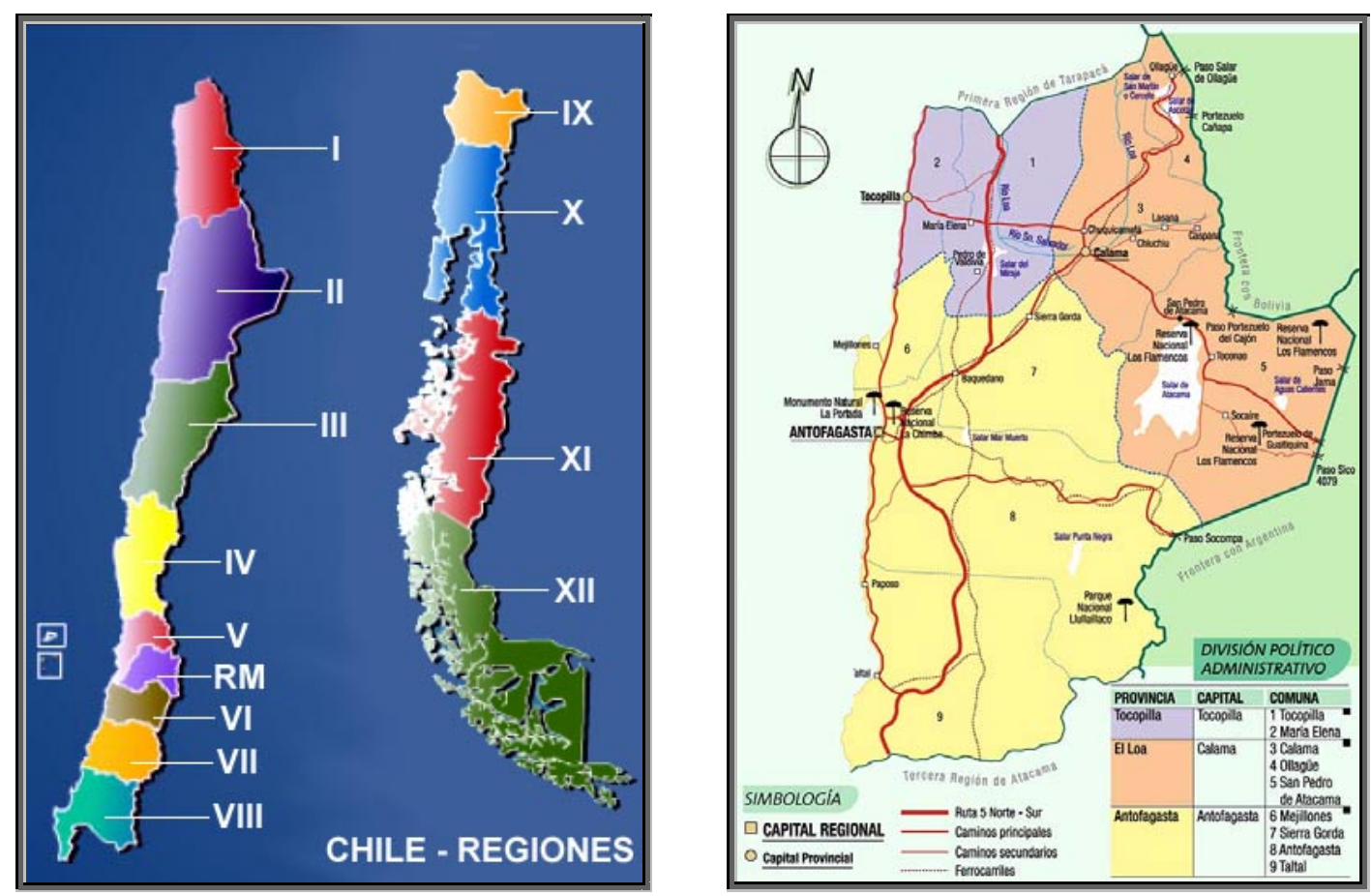

Figura 3.1: Zona de caminos en estudio - Región Antofagasta (II)

\subsection{APORTE DE LA BISCHOFITA EN CAMINOS NO PAVIMENTADOS}

La Bischofita es un estabilizador químico y agente de control de polvo para caminos no pavimentados. Se compone mayoritariamente de Cloruro de Magnesio Hexahidratado (Bischofita). Su apariencia es transparente, cristalina $y$ sin olor.

La Bischofita se produce de manera natural a partir de salmueras ricas en magnesio existentes en el Salar de Atacama. Estas salmueras son bombeadas y depositadas en pozas de evaporación solar donde cristaliza, proceso que es controlado en forma continua con el propósito de asegurar la calidad del producto. Su manera de funcionar es gracias a su alta higroscopicidad que permite atraer y retener la humedad, aminorando de este modo la pérdida de partículas finas del suelo y controlando la emisión de polvo. La Bischofita aglomera las partículas finas produciendo superficies de rodado duraderas. Además provee resistencia a la acción abrasiva del tránsito debido a la formación de una cubierta en la superficie del camino producida por su cristalización. 
Su forma de uso es diluyendo la Bischofita en agua formando una solución de cloruro de magnesio, pudiendo ser usado como estabilizador de capas de rodado (mezclándola homogéneamente con el suelo del camino) o como agente de control de polvo (riego sobre superficie del camino).

La Bischofita es utilizada con éxito en caminos no pavimentados y faenas mineras de Perú y Chile, y en centros urbanos y agrícolas del centro y norte del país. El Cloruro de Magnesio es usado en todo el mundo siendo Norteamérica el principal mercado. En esta región soluciones de cloruro de magnesio se utilizan con éxito en áreas ambientales sensibles como caminos del Servicio Forestal de los Estados Unidos.

La Bischofita además mejora la trabajabilidad de los suelos, reduce el contenido de humedad óptimo y en algunos casos aumenta la densidad de compactación.

\subsection{CRITERIO DE SELECCION DE CAMINOS}

Para seleccionar los caminos se ocuparon dos criterios; el primero está relacionado con la factibilidad de medir la rugosidad y el segundo la pérdida de material.

En ambos caso, los caminos seleccionados deberían cumplir con los siguientes requisitos:

a) Caminos pertenecientes a la red vial chilena sobre los cuales se tienen programas de inversión específica, cuya clasificación es la siguiente:

- Tipo A : Caminos nacionales que pertenecen a la red vial estructurante del País.

- Tipo B : Caminos Regionales Primarios que pertenecen a la red vial estructurante del País.

- Tipo C : Caminos Regionales Secundarios de importancia a nivel provincial. 
- Tipo D : Caminos Comunales Primarios de baja importancia como estructurante de los viajes.

- Tipo E : Caminos Comunales Secundarios de baja importancia como estructurante de los viajes.

En el estudio, sólo se incluyeron caminos de la clasificación A y B, por tratarse de caminos no pavimentados considerados en el programa de gobierno "Caminos Básicos 5000" que tiene como objetivo disminuir el mantenimiento y la contaminación ambiental por emanación de polvo a través de la aplicación de bischofita.

b) El estado de los caminos que se encontraron comprendió desde muy buenos hasta regulares.

c) Caminos que tuvieran censo de tránsito regular, con el objeto de tener posibilidades de comparar los resultados.

d) Transitabilidad durante todo el período de estudio.

e) Con distintos tránsitos medios diarios anuales (TMDA).

f) Para medir la Pérdida de Material, se requirió que los caminos tuviesen una capa granular superior a 5 centímetros de espesor.

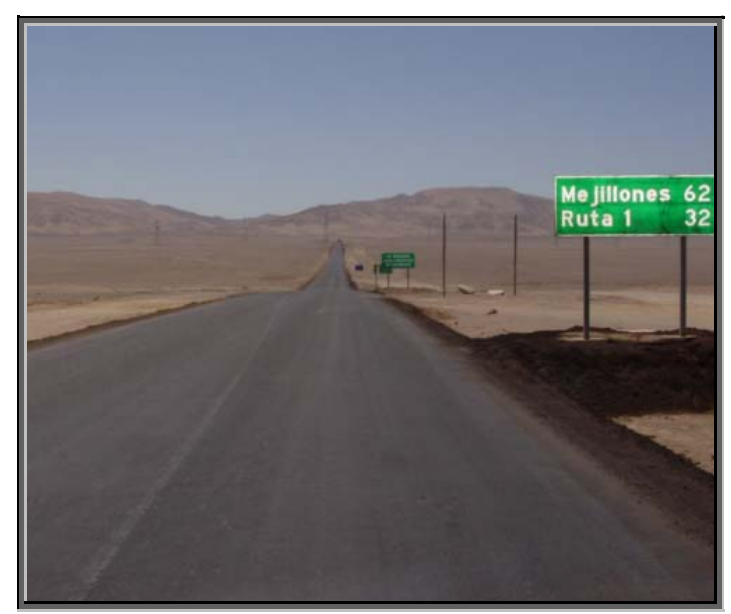

Ruta - B - 400

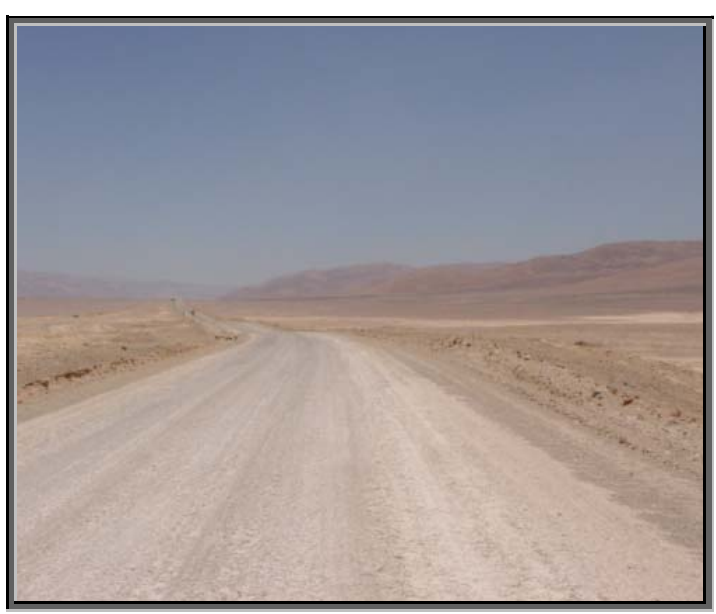

Ruta - B - 55

Figura 3.2: Caminos no pavimentados, estabilizados con bischofita - Región Antofagasta 
La nómina de los caminos elegidos que cumplían con las condiciones antes señaladas, se indican en las tablas siguientes:

Tabla 3.1: Caminos no pavimentados de la Región de Antofagasta seleccionados para medir rugosidad

\begin{tabular}{|l|c|l|l|l|}
\hline ROL & CLASE & \multicolumn{1}{|c|}{ REGIóN } & \multicolumn{1}{|c|}{ NOMBRE DEL CAMINo } & \multicolumn{1}{|c|}{ ZONA } \\
\hline 21-CH & A & Antofagasta & Calama - Ollagüe & Depresión Andina \\
\hline B-55 & B & Antofagasta & Cruce ruta 5 - Socompa & Depresión Intermedia \\
\hline B-155 & B & Antofagasta & Cruce ruta 21 - CH - Linzor & Depresión Andina \\
\hline B-165 & B & Antofagasta & Vado Rio Salado - Toconce & Depresión Andina \\
\hline B-169 & B & Antofagasta & Chiu-Chiu - Vado Rio Salado & Depresión Andina \\
\hline B-180 & B & Antofagasta & María Elena - Cruce Ruta 24 & Depresión Intermedia \\
\hline B-207 & B & Antofagasta & Cuesta Barros Arana - Rio Grande & Depresión Andina \\
\hline B-400 & B & Antofagasta & Estación Uribe - Guanaquitos & Cordillera de la Costa \\
\hline B-955 & B & Antofagasta & La Isla - Altamira & Depresión Intermedia \\
\hline
\end{tabular}

Tabla 3.2: Caminos no pavimentados de la Región de Antofagasta seleccionados para el estudio de pérdida de material

\begin{tabular}{|c|c|c|l|l|}
\hline ROL & CLASE & REGION & \multicolumn{1}{|c|}{ NOMBRE DEL CAMINO } & \multicolumn{1}{|c|}{ ZONA } \\
\hline B-55 & B & Antofagasta & Cruce ruta 5 - Socompa & Depresión Intermedia \\
\hline B-207 & B & Antofagasta & Cuesta Barros Arana - Rio Grande & Depresión Andina \\
\hline B-400 & B & Antofagasta & Estación Uribe - Guanaquitos & Cordillera de la Costa \\
\hline
\end{tabular}



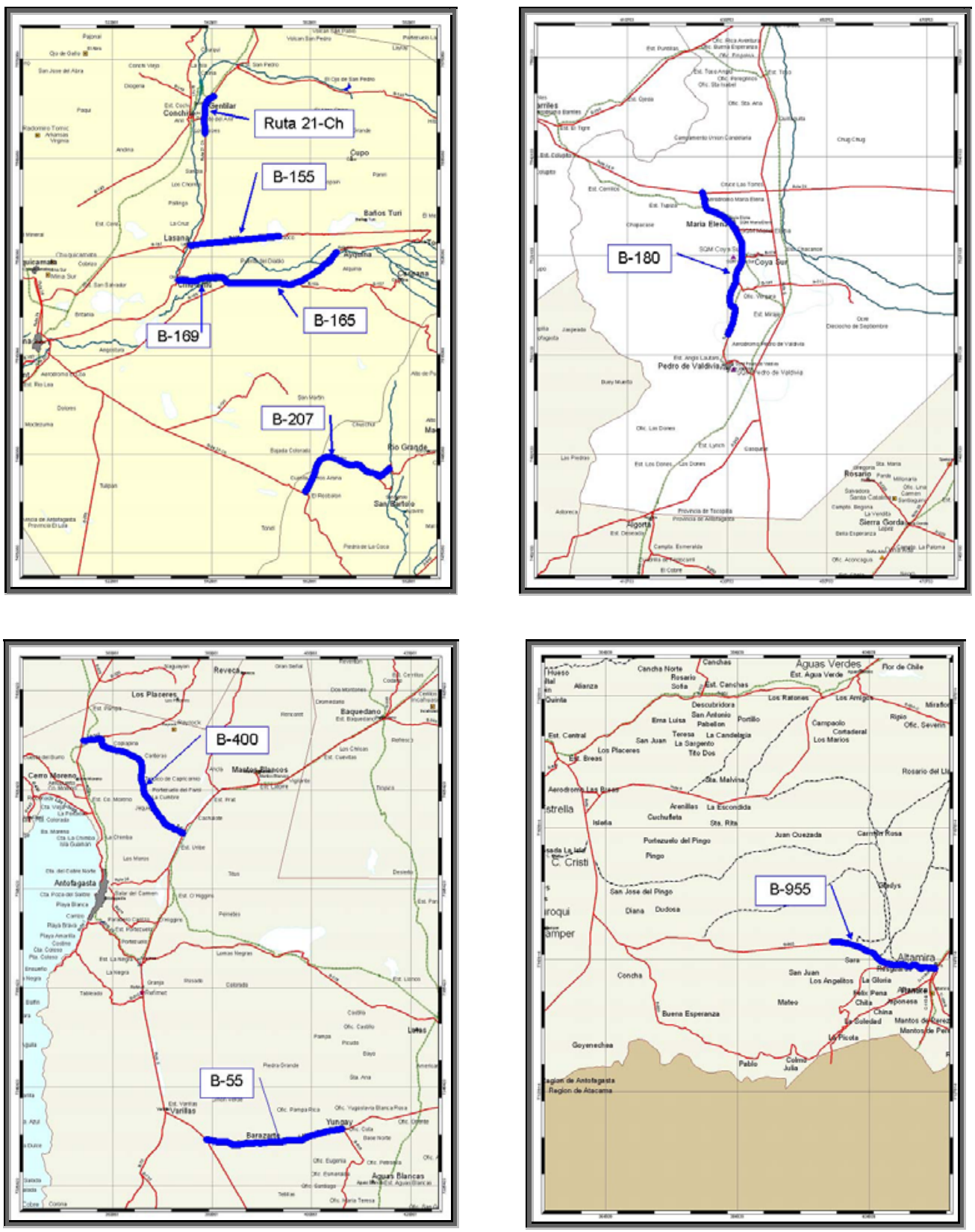

Figura 3.3: Ubicación geográfica de los caminos testigos

\subsection{CRITERIO DE SELECCION DE LOS TRAMOS TESTIGOS}

Junto con seleccionar los caminos, se seleccionaron los tramos testigos en cada camino. Para el estudio de la rugosidad el criterio ocupado fue el siguiente:

i) El largo del tramo recomendable es entre 500 y 3000 metros para facilitar las mediciones de rugosidad según las condiciones de cada 
camino, con lecturas de conteo cada 50, 100 ó 200 metros.

ii) Los puntos referenciales, tanto inicial como de fin de tramo, debían ser de fácil ubicación, en lo posible inmóviles en el tiempo y espacio, por ello se procedió a balizar los tramos.

iii) Homogeneidad del suelo de la capa a lo largo del tramo.

iv) Homogeneidad geométrica a lo largo del tramo; las zonas de curvas o rectas, tomarlas en lo posible en forma separada, evitando tramos con geometría mixta, fundamentalmente para evitar una posible distorsión en los valores medidos por efectos de una reducción de la velocidad media del tránsito medio diario en las zonas de curvas. Este criterio fue imposible de cumplir en la mayoría de los caminos.

v) El tramo no debería estar en zonas urbanas, ya que dificulta la medición de rugosidad, en especial para mantener la velocidad constante durante la medición.

Para determinar la Evolución de la Rugosidad en el tiempo se consideraron 18 tramos que se caracterizaron con las letras CR (Camino-Rugosidad), los cuales se detallan en la tabla 3.3.

El criterio ocupado para los tramos en que se midió la Pérdida de Material fue el siguiente:

i) Homogeneidad del suelo de la capa a lo largo del tramo

ii) Homogeneidad geométrica del tramo.

iii) Capa de grava superior a cinco centímetros.

iv) Largo aproximado de ciento veinte metros.

v) Con políticas de conservación conocidas.

En este caso, se eligieron nueve tramos que se caracterizaron con las letras CPM (Camino-Pérdida de Material), los cuales se detallan en la tabla 3.4. 
Tabla 3.3: Tramos elegidos para medir rugosidad

\begin{tabular}{|c|c|c|c|c|c|}
\hline Tramo & Ruta & TMDA & Provincia & Km - Inicial & Km. - Final \\
\hline CR1 & $21 C h$ & 66 & El Loa & 70,00 & 71,40 \\
\hline CR2 & $21 C h$ & 66 & El Loa & 71,50 & 72,90 \\
\hline CR3 & B-55 & 126 & Antofagasta & 15,60 & 18,60 \\
\hline CR4 & B-55 & 126 & Antofagasta & 22,00 & 25,00 \\
\hline CR5 & B-155 & 17 & El Loa & 1,00 & 4,00 \\
\hline CR6 & B-155 & 17 & El Loa & 4,50 & 7,50 \\
\hline CR7 & B-165 & 29 & El Loa & 37,00 & 39,00 \\
\hline CR8 & B-165 & 30 & El Loa & 39,00 & 41,00 \\
\hline CR9 & B-165 & 30 & El Loa & 41,00 & 43,00 \\
\hline CR10 & B-169 & 22 & El Loa & 3,00 & 6,00 \\
\hline CR11 & B-169 & 22 & El Loa & 7,00 & 10,00 \\
\hline CR12 & B-180 & 319 & Tocopilla & 13,00 & 16,00 \\
\hline CR13 & B-180 & 319 & Tocopilla & 25,00 & 28,00 \\
\hline CR14 & B-207 & 20 & El Loa & 6,30 & 8,80 \\
\hline CR15 & B-207 & 20 & El Loa & 9,00 & 11,50 \\
\hline CR16 & B-400 & 190 & Antofagasta & 1,00 & 5,00 \\
\hline CR17 & B-400 & 190 & Antofagasta & 6,00 & 10,00 \\
\hline CR18 & B-400 & 190 & Antofagasta & 16,00 & 20,00 \\
\hline CR19 & B-400 & 190 & Antofagasta & 21,00 & 25,00 \\
\hline CR20 & B-955 & 62 & Antofagasta & 43,00 & 46,00 \\
\hline CR21 & B-955 & 62 & Antofagasta & 46,00 & 49,00 \\
\hline
\end{tabular}

Tabla 3.4: Tramos elegidos para medir pérdida de material

\begin{tabular}{|c|c|c|c|c|c|}
\hline Tramo & Ruta & TMDA & Provincia & Km - Inicial & Km. - Final \\
\hline CPM1 & B-55 & 131 & Antofagasta & 13,45 & 13,57 \\
\hline CPM2 & B-55 & 131 & Antofagasta & 13,60 & 13,72 \\
\hline CPM3 & B-55 & 131 & Antofagasta & 13,80 & 13,92 \\
\hline CPM4 & B-207 & 20 & El Loa & 10,66 & 10,78 \\
\hline CPM5 & B-207 & 20 & El Loa & 10,80 & 10,92 \\
\hline CPM6 & B-207 & 20 & El Loa & 11,00 & 11,12 \\
\hline CPM7 & B-400 & 190 & Antofagasta & 25,00 & 25,12 \\
\hline CPM8 & B-400 & 190 & Antofagasta & 25,20 & 25,32 \\
\hline CPM9 & B-400 & 190 & Antofagasta & 25,40 & 25,52 \\
\hline
\end{tabular}




\subsection{DISEÑO DEL ESTUDIO}

Para determinar la importancia de los factores que inciden en el deterioro de los caminos no pavimentados estabilizados con bischofita, se abocó en forma especial a medir la evolución tanto de rugosidad como de pérdida de material que presentaban los tramos escogidos en un período de tiempo.

\subsubsection{Evolución de la Rugosidad}

Para determinar la evolución de la rugosidad a través del tiempo, se trabajó con la base de datos de la Dirección Regional de Vialidad más una medición en campo en el período en estudio.

El procedimiento de medición consistió en pasar un rugosímetro MIS4 a una velocidad constante de $50 \mathrm{kph}$ una vez por sentido, midiendo la regularidad superficial de la huella externa.

El rugosímetro MIS4 es un medidor de Regularidad Superficial del tipo respuesta, que permite la recolección y presentación de la regularidad del pavimento o IRI. Su principio de funcionamiento está basado directamente en la evaluación de la respuesta dinámica de un vehículo, es decir, mide las aceleraciones verticales acumuladas percibidas por la suspensión del vehículo al recorrer la superficie de rodado.

El equipo va montado en un vehículo acondicionado para la instalación de un acerelerómetro, un odómetro y un computador que procesa la información.

\subsubsection{Evolución de la Pérdida de Material}

Para determinar la evolución de la Pérdida de Material a través del tiempo se procedió a medir ésta en forma regular durante un período de 6 meses, mediante dos métodos: "Calicatas y Topográfico".

El método topográfico se implementó fundamentalmente para evitar el deterioro de las capas de rodado con la perforación de calicatas, aumentar el número de registros y mejorar la precisión de las mediciones realizadas por el "Método Calicatas". 


\section{i) Método de Calicatas}

Este método pretende determinar la Pérdida de Material a través de la medición del espesor remanente de la capa, es decir, medir la disminución o evolución del espesor de la capa a través del tiempo, y así poder estimar la Pérdida de Material en un año.

El procedimiento consistió en la realización de calicatas, cuyas dimensiones eran $40 \mathrm{~cm}$ de largo por $30 \mathrm{~cm}$ de ancho y una altura variable entre 10 y $40 \mathrm{~cm}$ dependiendo del espesor de la capa.

Las mediciones se realizaron cada 45 días por un periodo de seis meses, extrapolando linealmente para un año los valores obtenidos en las mediciones. Se hicieron un total de siete calicatas por tramo según la distribución que muestra la figura 3.4.

\begin{tabular}{|l|c|c|c|c|c|c|c|}
\hline \multicolumn{1}{|c|}{ Km } & 0.000 & 0.020 & 0.040 & 0.060 & 0.080 & 0.100 & 0.120 \\
\hline $\begin{array}{l}\text { Huella externa } \\
\text { pista Derecha }\end{array}$ & 0 & & & & 0 & & \\
\hline Eje & & 0 & & 0 & & 0 & \\
\hline $\begin{array}{l}\text { Huella externa } \\
\text { pista Izquierda }\end{array}$ & & & 0 & & & & 0 \\
\hline
\end{tabular}

Figura 3.4: Esquema de ubicación de las calicatas

Por tratase de calicatas relativamente rectangulares era posible medir el espesor de la capa en sus cuatro costados y así obtener un valor promedio por calicata, el cual era nuevamente promediado con los valores de las calicatas restantes, obteniéndose un valor más representativo del espesor remanente de la capa y por consiguiente de la pérdida de Material.

\section{ii) Método Topográfico}

Este método trata de utilizar las ventajas de la topografía para medir la 
Pérdida de Material, la cual consiste en efectuar el método usual de nivelación, es decir, conocer los desniveles y cotas respectivas de ciertos puntos de interés con respecto a una cota dada materializada en terreno.

Se logró hacer una medición topográfica cada 45 días por un período de seis meses, extrapolando linealmente para un año los valores obtenidos en la medición. Todas las mediciones se realizaron con la ayuda del Laboratorio Regional de Vialidad.

El procedimiento del Método Topográfico consistió en hacer una nivelación de cada uno de los tramos medidos por el Método de Calicatas, con tres puntos en el ancho de la ruta. La nivelación topográfica, se realizó el mismo día de la medición de las calicatas, ésta pretende verificar la variación del nivel superficial de la capa y en base a ello determinar la Pérdida de Material. En la nivelación topográfica se utilizó como instrumento un taquímetro digital de alta precisión. Ante la imposibilidad de balizar los puntos de medición con algún tipo de pintura, de tal forma de tener la certeza de que las futuras mediciones se realizarán sobre el mismo punto, sólo se optó por referenciar las mediciones en base al borde de las calicatas, trazando un línea separada $10 \mathrm{~cm}$ del borde de la calicata, perpendicular al eje del camino. Sobre la línea se trazaban los puntos: huella externa pista derecha, eje y huella externa pista izquierda.

\begin{tabular}{|c|c|c|c|c|c|c|c|}
\hline $\mathrm{Km}$ & 0.000 & 0.020 & 0.040 & 0.060 & 0.080 & 0.100 & 0.120 \\
\hline $\begin{array}{l}\text { Huella externa } \\
\text { pista Derecha }\end{array}$ & 0 & 0 & 0 & 0 & 0 & 0 & 0 \\
\hline Eje & 0 & 0 & 0 & 0 & 0 & 0 & 0 \\
\hline $\begin{array}{l}\text { Huella externa } \\
\text { pista Izquierda }\end{array}$ & 0 & 0 & 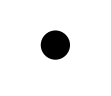 & 0 & 0 & 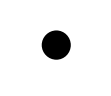 & 0 \\
\hline
\end{tabular}

Figura 3.5: Esquema de nivelación topográfica 


\subsection{VARIABLES MEDIDAS EN EL ESTUDIO}

\subsubsection{Variables Geométricas}

- LARGO: Se adoptó como largo mínimo 500 m y máximo de 3000 m.

- PENDIENTE: Las pendientes longitudinales medidas en los tramos seleccionados variaban desde nula a pendiente media del $3 \%$.

- RADIO DE CURVATURA HORIZONTAL: En general se seleccionaron tramos con poca curvatura.

- ANCHO DE VIA: Los anchos de vía de los tramos variaron entre 5 y $9 \mathrm{~m}$.

Tabla 3.5: Variables medidas en el estudio

\begin{tabular}{|c|c|c|c|c|}
\hline \multirow{2}{*}{ VARIABLES } & \multirow{2}{*}{ MEDIA } & \multirow{2}{*}{$\begin{array}{l}\text { DESV. } \\
\text { STANDARD }\end{array}$} & \multicolumn{2}{|c|}{ LÍMITES } \\
\hline & & & MÍNIMO & MÁXIMO \\
\hline \multicolumn{5}{|l|}{ NUMERO DE TRAMOS = 21} \\
\hline PENDIENTE ( RF - m/km) & 10,9 & 10,33 & 0,5 & 33,0 \\
\hline CURVATURA (HC - \%/km) & 35,9 & 36,76 & 0,0 & 128,8 \\
\hline LARGO DE TRAMOS(m) & 2.594 & 723,94 & 600 & 3.000 \\
\hline ANCHO DE LA VIA (m) & 7,00 & 1,07 & 6,00 & 9,00 \\
\hline PRECIPITACION (mm/mes) & 0,68 & 0,67 & 0,14 & 1,43 \\
\hline \multicolumn{5}{|c|}{ CARACTERISTICAS DEL MATERIAL } \\
\hline - \%" QUE PASA POR MALLA 10 & 51 & 6,98 & 40 & 69 \\
\hline - \%- QUE PASA POR MALLA 40 & 33 & 5,25 & 22 & 44 \\
\hline -\%" QUE PASA POR MALLA 200 & 17 & 5,80 & 8 & 30 \\
\hline -INDICE DE PLASTICIDAD (\%") & 5 & 2,70 & 1,50 & 10 \\
\hline -LIMITE LIQUIDO(\%) & 24 & 5,30 & 17 & 35 \\
\hline \multicolumn{5}{|c|}{ TRANSITO MEDIO DIARIO (en los dos sentidos) } \\
\hline - AUTOMOVILES & 16 & 31,20 & 1 & 101 \\
\hline - BUSES & 6 & 10,72 & 1 & 35 \\
\hline - CAMIONETAS & 33 & 35,31 & 10 & 124 \\
\hline - CAMIONES LIVIANOS & 9 & 8,70 & 1 & 24 \\
\hline - CAMIONES PESADOS & 31 & 35,66 & 0 & 108 \\
\hline \multicolumn{5}{|c|}{ INFORMACION SOBRE PÉRDIDA DE GRAVA } \\
\hline \multicolumn{5}{|l|}{ - Número de Observaciones $=27$} \\
\hline - Perdida anual de material (mm) & 27.5 & 11.7 & 12 & 45 \\
\hline \multicolumn{5}{|c|}{ INFORMACION SOBRE MEDIDAS DE RUGOSIDAD } \\
\hline \multicolumn{5}{|l|}{ - Número de Observaciones $=600$} \\
\hline - Rugosidad medida (IRI) & 5,0 & 1,94 & 2,0 & 14,0 \\
\hline
\end{tabular}

Fuente: elaboración propia 
Modelo en alzado para calcular el promedio de subidas y bajadas (RF)

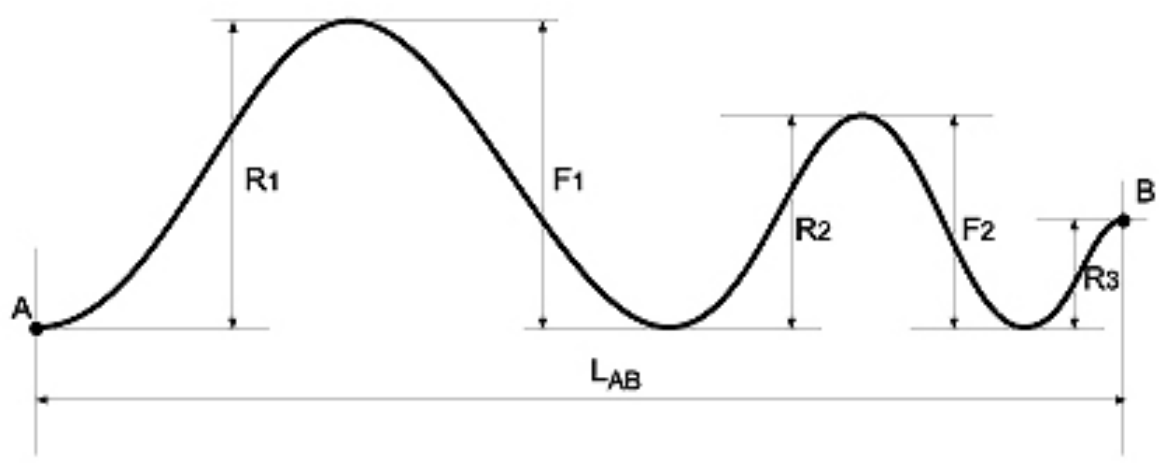

$$
R F=\frac{R_{1}+R_{2}+R_{3} \cdots+R_{n}+F_{1}+F_{2} \cdots+F_{n}}{L_{A B}} \underset{(\mathrm{Km})}{(m)}
$$

Modelo en planta para calcular el promedio de curvatura horizontal (C)

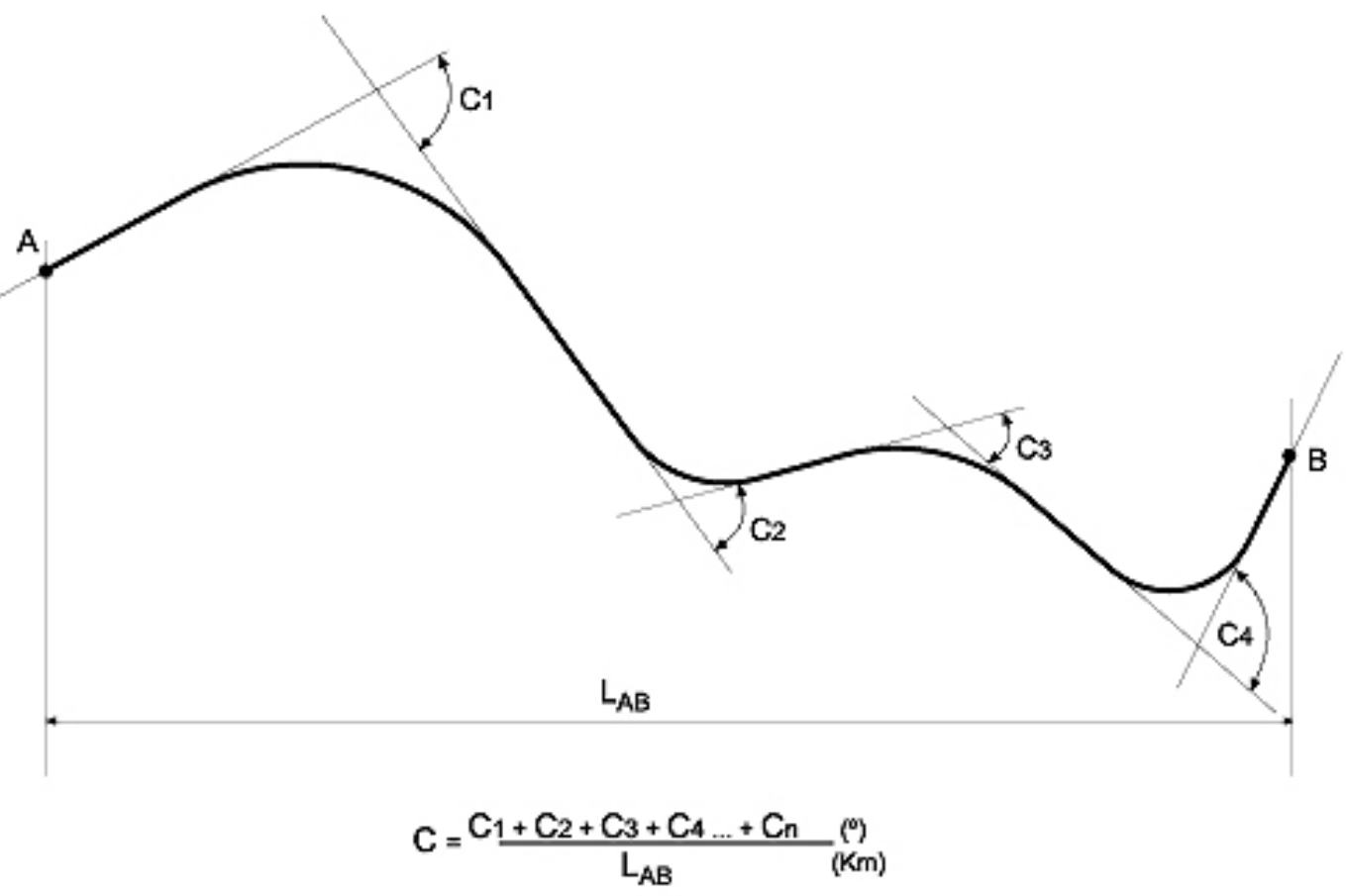

Figura 3.6: Ilustración subida y bajadas "RF" y curvatura horizontal "HC" 


\subsubsection{Variables Geotécnicas}

Las variables geotécnicas que se estudiaron son las que requieren los modelos, y corresponden a las características del material de la capa de rodadura, ya que según el estudio brasileño, para efectos del deterioro, no se consideran las características de la sub-base si la hubiese.

- GRANULOMETRIA: Tamaño máximo del material granular (mm).

- Porcentaje de material que pasa tamiz 10.

- Porcentaje de material que pasa tamiz 40.

- $\quad$ Porcentaje de material que pasa tamiz 200.

- $\quad$ LIMITES DE ATTERBERG: Límite Líquido e Indice de Plasticidad

- ESPESOR DE LA CAPA DE RODADURA: en mm.

- DENSIDAD EN TERRENO DE LA CAPA: en $\mathrm{Kg} / \mathrm{cm}^{3}$.

- RAZON DE SOPORTE DE CALIFORNIA (CBR)

\subsubsection{Variables Ambientales:}

\section{Altura sobre el nivel del mar:}

Según el HDM-IV ésta corresponde a una variable ambiental. La región de Antofagasta se caracteriza por tener cinco zonas geográficas básicas, cada una de las cuales cuenta con características climáticas y ecológicas propias.

Estas son: la costa y las planicies litorales (altura media $70 \mathrm{msnm}$ ), la zona de la Cordillera de la Costa (con cerros que sobrepasan los $2.000 \mathrm{msnm}$ ), la Depresión Intermedia (altura media de $1.400 \mathrm{msnm}$ ), las serranías del brazo occidental de la Cordillera de los Andes o Depresión Andina (alturas del orden de los $2.500 \mathrm{msnm}$ ) y el altiplano o Cordillera de los Andes (alturas del orden de los $6.000 \mathrm{msnm})$. Además, al este de la Depresión Intermedia nace un nuevo accidente geográfico denominado Cordillera de Domeyko (4.300 msnm). 


\section{Cantidad de agua caída:}

Las características climáticas de la región de Antofagasta son de una marcada aridez. El desierto se manifiesta plenamente hacia la zona intermedia, donde la influencia marítima, propia del relieve pierde importancia. La situación de extrema aridez en la depresión intermedia y la escasa vegetación existente, definen un paisaje natural conocido como desierto de Atacama. En esta región se localizan cuatros subtipos climáticos desérticos, localizados en franjas longitudinales ${ }^{1}$.

- Clima desértico costero nubloso: se localiza a lo largo de la costa, sus efectos se manifiestan hasta 20 kilómetros hacia el interior, donde la sequedad atmosférica es mayor, debido a que por causas del relieve, la influencia marítima es retenida en los cerros de la Cordillera de la Costa. Las características principales de este subtipo climático se traduce en un efecto modelador de las temperaturas, produciendo la corriente fría de Humboldt la presencia de abundante humedad, neblinas matinales y la ausencia de precipitaciones. Las lluvias registran un leve aumento hacia el sur del litoral, de igual manera lo mismo ocurre hacia el interior del altiplano de la región.

- Clima desértico interior: se desarrolla en la franja intermedia de la región y corresponde al clima desértico propiamente tal, caracterizado por una aridez extrema, ausencia de humedad, gran sequedad atmosférica y una gran amplitud térmica entre el día y la noche, las temperaturas diurnas extremas superan los $30^{\circ} \mathrm{C}$ y en la noche descienden a $1^{\circ} \mathrm{C}$ o menos. En las zonas intermedias de las pampas interiores encerradas por las serranías del oriente de la precordillera Andina, posee las características climáticas mas áridas del norte chileno, en el desierto de Atacama, las precipitaciones son muy escasas y la humedad relativa es inferior al $25 \%$.

\footnotetext{
${ }^{1}$ Inzunza B. Juan Carlos. "Climas de Chile. Capitulo 15 “. Universidad de Concepción de Chile. http://www2.udec.cl/ iinzunza/meteo/meteo.htm
} 
- Clima desértico marginal de altura: se localiza entre los 2000 y 3500 metros sobre el nivel del mar, el cual presenta mayor cantidad de volumen de precipitaciones en los meses de verano, con valores entre 20 y $60 \mathrm{~mm}$ anuales, esto permite el asentamiento de poblados cordilleranos como San Pedro de Atacama, Toconao, Chiu-Chiu entre otros.

- Clima de estepa de altura: se localiza preferentemente en las áreas de los bordes o márgenes de los desiertos. En esta región se ubica por sobre los $3500 \mathrm{~m}$ sobre el nivel del mar lo que corresponde a la zona altiplánica o Puna, sus principales características son las bajas temperaturas, siendo la media anual de $2^{\circ} \mathrm{C}$, la amplitud térmica entre el día y la noche es muy alta, superior a $20^{\circ} \mathrm{C}$. Las precipitaciones se producen en los meses de verano y no sobrepasan los $100 \mathrm{~mm}$ anuales, a medida que se avanza hacia el sur del altiplano de esta región, las lluvias de verano comienzan a disminuir y a mayor altura predomina una precipitación sólida.

La cantidad de agua lluvia caída en la zona de estudio y durante el período en que éste duró (2005-2006), se obtuvo de la Dirección Meteorológica de Chile y de la Dirección de Aguas del Ministerio de Obras Públicas. Respecto de aquellas zonas que no cuentan con registros por no ubicarse una estación meteorológica en las inmediaciones, la pluviometría promedio anual se estimó contrastando un Mapa de Isoyetas (isohietas) de Precipitaciones de la World Meteorological Organization (WMO) y los valores medios registrados por las estaciones meteorológicas de la región.

En los cuadros siguientes se establecen los datos pluviométricos registrados en las estaciones meteorológicas del Norte Grande de Chile, que comprende a las regiones de Tarapacá, Antofagasta y Atacama y aquellas utilizadas en el estudio: 
Tabla 3.6: Ubicación de estaciones pluviométricas en el norte de Chile

\begin{tabular}{|l|l|c|c|c|}
\hline \multicolumn{1}{|c|}{ REGIÓN } & \multicolumn{1}{|c|}{ ESTACION } & $\begin{array}{c}\text { LATITUD } \\
\text { SUR }\end{array}$ & $\begin{array}{c}\text { LONGITUD } \\
\text { OESTE }\end{array}$ & $\begin{array}{c}\text { ALTURA } \\
\text { msnm }\end{array}$ \\
\hline Tarapacá (I) & Arica & $18^{\circ} 20^{\prime} 58^{\prime \prime}$ & $70^{\circ} 20^{\prime} 11^{\prime \prime}$ & 58 \\
\hline Tarapacá (I) & Iquique & $20^{\circ} 32^{\prime} 50^{\prime \prime}$ & $70^{\circ} 10^{\prime} 44^{\prime \prime}$ & 52 \\
\hline Tarapacá (I) & Central Chapiquiña & $18^{\circ} 20^{\prime} 09^{\prime \prime}$ & $69^{\circ} 30^{\prime} 03^{\prime \prime}$ & 3.300 \\
\hline Antofagasta (II) & Ollagüe & $21^{\circ} 13^{\prime} 29^{\prime \prime}$ & $68^{\circ} 15^{\prime} 19^{\prime \prime}$ & 3.700 \\
\hline Antofagasta (II) & Lequena & $21^{\circ} 11^{\prime} 52^{\prime \prime}$ & $68^{\circ} 40^{\prime} 03^{\prime \prime}$ & 3.300 \\
\hline Antofagasta (II) & Embalse Conchi & $22^{\circ} 01^{\prime} 28^{\prime \prime}$ & $68^{\circ} 37^{\prime} 06^{\prime \prime}$ & 3.100 \\
\hline Antofagasta (II) & Calama & $22^{\circ} 29^{\prime} 43^{\prime \prime}$ & $68^{\circ} 54^{\prime} 30^{\prime \prime}$ & 2.250 \\
\hline Antofagasta (II) & Antofagasta & $23^{\circ} 27^{\prime} 02^{\prime \prime}$ & $70^{\circ} 26^{\prime} 27^{\prime \prime}$ & 130 \\
\hline Atacama (III) & Copiapó & $27^{\circ} 17^{\prime} 56^{\prime \prime}$ & $70^{\circ} 24^{\prime} 52^{\prime \prime}$ & 302 \\
\hline Atacama (III) & Embalse Lautaro & $27^{\circ} 33^{\prime} 52^{\prime \prime}$ & $70^{\circ} 14^{\prime} 39^{\prime \prime}$ & 640 \\
\hline Atacama (III) & Vallenar & $28^{\circ} 35^{\prime} 38^{\prime \prime}$ & $70^{\circ} 45^{\prime} 27^{\prime \prime}$ & 535 \\
\hline
\end{tabular}

Tabla 3.7: Ubicación de estaciones pluviométricas asimiladas al estudio

\begin{tabular}{|l|c|l|l|l|c|}
\hline \multicolumn{1}{|c|}{ Región } & Rol - Ruta & \multicolumn{1}{|c|}{ ESTACION } & $\begin{array}{c}\text { LATITUD } \\
\text { SUR }\end{array}$ & $\begin{array}{c}\text { LONGITUD } \\
\text { OESTE }\end{array}$ & $\begin{array}{c}\text { ALTURA } \\
\text { msnm }\end{array}$ \\
\hline Antofagasta & $21-\mathrm{CH}$ & Embalse Conchi & $22^{\circ} 01^{\prime} 28^{\prime \prime}$ & $68^{\circ} 37^{\prime} 06^{\prime \prime}$ & 3.100 \\
\hline Antofagasta & B-55 & Antofagasta & $23^{\circ} 27^{\prime} 02^{\prime \prime}$ & $70^{\circ} 26^{\prime} 27^{\prime \prime}$ & 130 \\
\hline Antofagasta & B-155 & Embalse Conchi & $22^{\circ} 01^{\prime} 28^{\prime \prime}$ & $68^{\circ} 37^{\prime} 06^{\prime \prime}$ & 3.100 \\
\hline Antofagasta & B-165 & Embalse Conchi & $22^{\circ} 01^{\prime} 28^{\prime \prime}$ & $68^{\circ} 37^{\prime} 06^{\prime \prime}$ & 3.100 \\
\hline Antofagasta & B-169 & Embalse Conchi & $22^{\circ} 01^{\prime} 28^{\prime \prime}$ & $68^{\circ} 37^{\prime} 06^{\prime \prime}$ & 3.100 \\
\hline Antofagasta & B-180 & Antofagasta & $23^{\circ} 27^{\prime} 02^{\prime \prime}$ & $70^{\circ} 26^{\prime} 27^{\prime \prime}$ & 130 \\
\hline Antofagasta & B-207 & Calama & $22^{\circ} 29^{\prime} 43^{\prime \prime}$ & $68^{\circ} 54^{\prime} 30^{\prime \prime}$ & 2.250 \\
\hline Antofagasta & B-400 & Antofagasta & $23^{\circ} 27^{\prime} 02^{\prime \prime}$ & $70^{\circ} 26^{\prime} 27^{\prime \prime}$ & 130 \\
\hline Antofagasta & B-955 & Antofagasta & $23^{\circ} 27^{\prime} 02^{\prime \prime}$ & $70^{\circ} 26^{\prime} 27^{\prime \prime}$ & 130 \\
\hline
\end{tabular}




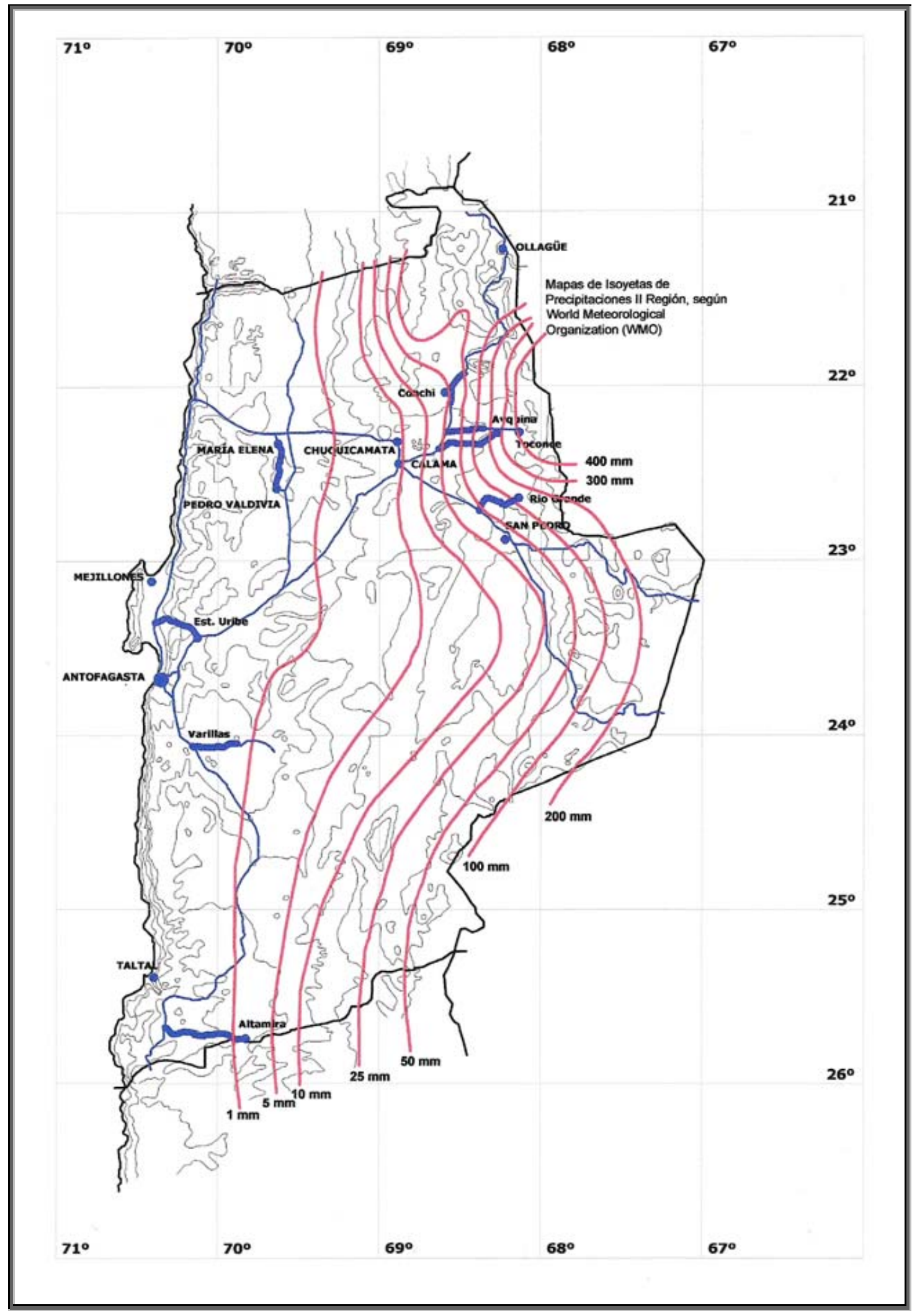

Figura 3.7: Mapa de Isoyetas de Precipitaciones de la World Meteorological Organization (WMO) 


\subsubsection{Variables de Tránsito}

Esta variable corresponde al Tránsito Medio Diario Anual que poseen los caminos en observación. Nos basamos en los censos nacionales de Vialidad de los años 2002 y en especial el del año 2004.

Para tal efecto los vehículos se clasificaron de la siguiente manera:

- AUTOS - STATION: Vehículo destinado al transporte de personas, cuya capacidad máxima es de nueve pasajeros.

- CAMIONETAS - FURGONES: Vehículo liviano usado para el movimiento de personas y pequeñas cargas.

- CAMIONES DE 2 EJES: Vehículo automotor de operación libre, destinado al transporte de carga.

- CAMIONES DE MÁS DE 2 EJES: En esta clasificación se han incluido a los camiones de más de dos ejes, a los vehículos remolcables (semitráiler) cuyo peso y carga se transmiten al camión que lo remolca, y a los tráileres (remolques) que son vehículo de uno, dos o más ejes simples o en tándem sin tracción propia, cuyo peso total descansa sobre sus propios ejes.

- BUSES: Vehículo automotor destinado al transporte colectivo de pasajeros con capacidad para más de 21 pasajeros y que habitualmente realizan viajes interregionales. Se incluyen también taxibuses y microbuses. 


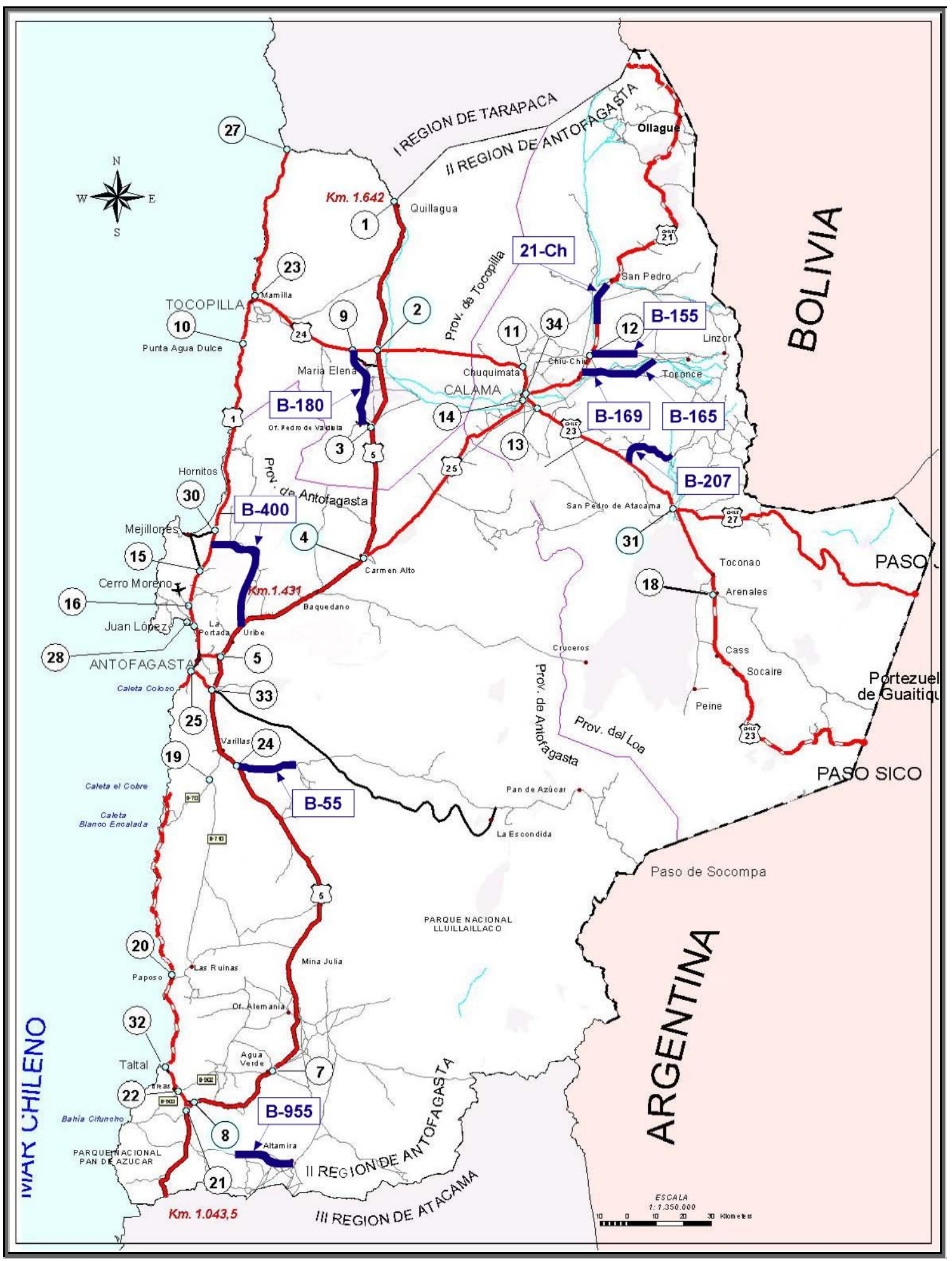

Figura 3.8: Ubicación de puntos censales de la Región de Antofagasta 


\subsection{EQUIPOS E INSTRUMENTOS UTILIZADOS}

\subsubsection{Equipos de Características Geométricas}

En esta oportunidad no fue necesario realizar la medición de estas características (pendiente, radio de curvatura y altura sobre el nivel del mar), ya que la Dirección Regional de Vialidad cuenta con una base de datos al respecto.

No obstante, Vialidad Regional utiliza para medir estas características un sistema GPS Pathfinder. Estos sistemas son muy efectivos en la captura, actualización y procesamiento de datos. El sistema se compone de un software, colectores de datos y receptores GPS. Son simples de usar y de fácil integración con las bases de datos GIS.

\subsubsection{Equipo de Medición de Rugosidad}

Para medir la rugosidad de los caminos se utilizó el rugosímetro MIS4, medidor de Regularidad Superficial del tipo respuesta, de fabricación chilena, que permite la recolección y presentación de la regularidad del pavimento o IRI. Su principio de funcionamiento está basado directamente en la evaluación de la respuesta dinámica de un vehículo. Según lo anterior, el equipo se define como rugosímetro del tipo respuesta o clase 3 , de acuerdo a la clasificación del Banco Mundial.

De acuerdo con la clasificación del Banco Mundial los métodos para la medición de la rugosidad se agrupan en 4 clases, siendo los de Clase 1 los más exactos (mira y nivel, viga TRRL, perfilómetros estáticos como el MERLIN). La Clase 2 agrupa a los métodos que utilizan los perfilómetros estáticos y dinámicos, pero que no cumplen con los niveles de exactitud que son exigidos para la Clase 1. Los métodos Clase 3 utilizan ecuaciones de correlación para derivar sus resultados a la escala del IRI (Bump integrator, Mays meter). Los métodos Clase 4 permiten obtener resultados meramente referenciales y se emplean cuando se requieren únicamente estimaciones gruesas de la rugosidad. 
Este instrumento mide las aceleraciones verticales acumuladas percibidas por la suspensión del vehículo al recorrer la superficie de rodado (huella externa de la pista).

Este equipo va montado en un vehículo especialmente acondicionado para la instalación de un acerelerómetro, un odómetro y un computador de proceso de la información.
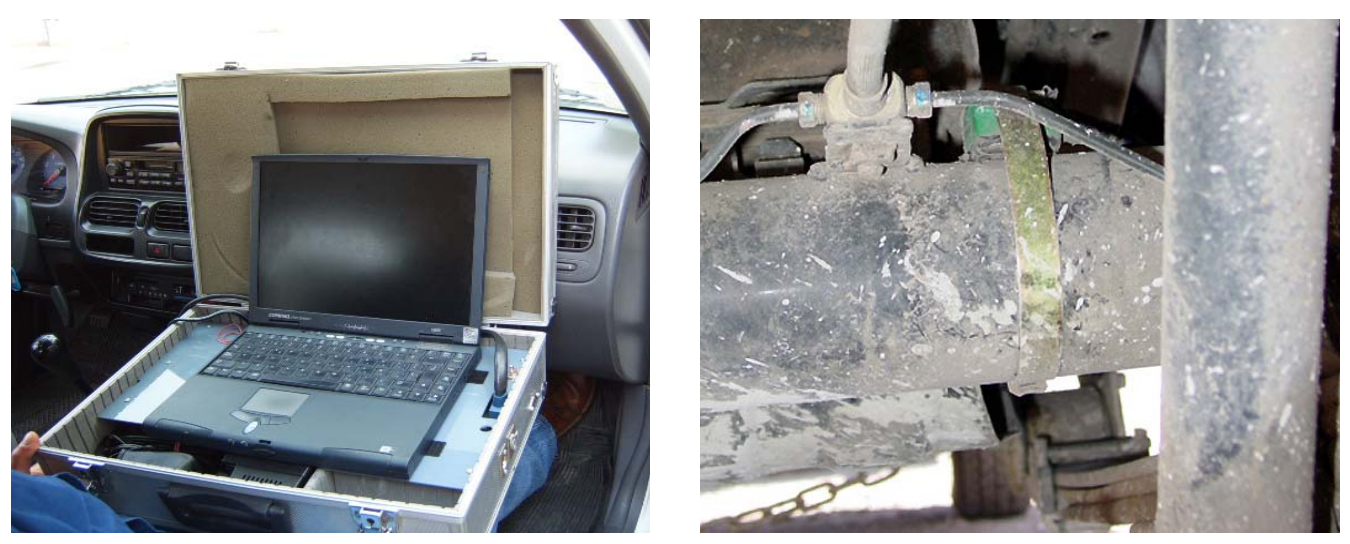

Figura 3.9: Unidad de recolección de datos y acelerómetro

Las aceleraciones obtenidas son correlacionadas con el Índice de Regularidad Internacional IRI, obteniéndose de ésta manera mediante software los valores de IRI en tiempo real.

La simpleza de este equipo permite evaluar la regularidad superficial de los caminos de bajo estándar sin problemas de operación. Los valores de IRI obtenidos tienen una correlación aceptable con los determinados a partir de un equipo con sensores láser, lo que permitiría medir sin mayores problemas la red pavimentada del país.

El equipo consta básicamente de tres componentes:

i) Acelerómetro. Dispositivo que mide la respuesta a los desplazamientos verticales acumulados por unidad de distancia.

ii) Odómetro. Dispositivo para determinar la distancia de recorrido.

iii) Sistema automático de adquisición, almacenamiento y procesamiento de la información, que además permite almacenar dicha información en 
medio digital para su posterior transferencia a bancos de datos.

El éxito de este experimento depende de la confiabilidad y calidad de la información recolectada por el equipo, ésta debe permitir obtener un IRI con características de repetibilidad y correlación con respecto a equipos de precisión clase I, según clasificación del Banco Mundial (perfilómetros tipo láser u ópticos). La desviación estándar de las mediciones de IRI en un mismo sector de 100 metros debe ser menor a $0.1 \mathrm{~m} / \mathrm{km}$., con un coeficiente de correlación $\mathrm{R}^{2} \geq 0.80$.

\section{- Calibración del Rugosímetro MIS4}

La calibración consistió en la medición simultánea de la regularidad superficial con un Perfilómetro Láser y con el MIS4, en tramos de 1,5 km de longitud de ruta con distintos IRI.

La medición para calibración con equipo tipo respuesta se efectuó a las velocidades posibles de medición, que pueden ser 40, 50, 60 ó 70 kph. Se realizaron tres repeticiones por velocidad, para verificar la repetibilidad del equipo. Luego de procesar los valores de IRI medidos con equipo Clase I, se contrastaron con el promedio de las aceleraciones verticales obtenidas a partir de la respuesta dinámica del vehículo en un intervalo de $200 \mathrm{~m}$.

El factor de calibración se determinó a partir de la relación lineal que se obtuvo entre las aceleraciones promedio y el valor de IRI. Obteniendo un factor de calibración para cada velocidad de medición.

Es importante tener presente que antes de cada campaña de medición se debe verificar el factor de calibración a aplicar, efectuando mediciones en los tramos de control definidos para ello.

\section{- Calibración del Odómetro incorporado al Rugosímetro}

El odómetro, de fabricación chilena, se conecta al sistema eléctrico del vehículo. Mediante pulsos generados en un sensor que se instala 
directamente al cuentakilómetros, el instrumento contabiliza las revoluciones de éste, lo que se traduce en distancias.

Este instrumento también debe calibrarse en cada operativo, para ello se cuenta con pistas en la Ruta 5 Norte, con la que se obtiene el valor de conversión de pulsos del odómetro a metros.

\subsubsection{Equipo de medición Características Geotécnicas}

Las características geotécnicas de los caminos se obtuvieron de la base de datos del Laboratorio Regional de Vialidad (LRV) y para los datos faltantes se emplearon los equipos del mismo laboratorio. Además para determinar la Densidad en terreno se empleó un densitómetro nuclear.

\subsubsection{Equipo de medición de Pérdida de Material}

Los elementos empleados para medir la pérdida de material consistieron en un pico y una pala para la confección de las calicatas y un vernier para la medición del espesor de la capa.

En cuanto a las mediciones topográficas estas fueron hechas a través de un taquímetro digital, odómetro, cinta metálica y estacas de madera.

\subsection{TOMA DE DATOS}

\subsubsection{Medición de Rugosidad}

Para realizar un seguimiento completo de los caminos no pavimentados del área de estudio era deseable que se midiese la rugosidad como mínimo una vez al mes en un período de tres meses, lo que no fue posible de realizar por no contar con la disponibilidad de los equipos de propiedad del Laboratorio Nacional de Vialidad. Sin embargo, se logró medir la rugosidad en cada tramo en una oportunidad. Además se contó con una base de datos que establece registros de dos mediciones anteriores, lográndose conformar una serie histórica sólo de algunas rutas, situación que queda señalada en las tablas de datos del capítulo cuarto. 
Las campañas de medición de los caminos considerados en el estudio y almacenadas en las bases de datos de la Dirección Regional de Vialidad, se practicaron durante los años 2003, 2004 y 2005. Realizando una última medición durante el período de esta investigación, enero del 2006.

\subsubsection{Ensayes de Suelos}

Estos fueron realizados por la Sección de Geotecnia del LNV en conjunto con el Laboratorio Regional de Vialidad de Antofagasta (LRV) durante un período comprendido entre octubre del 2003 y mayo del 2004.

\subsubsection{Mediciones de Pérdida de Material}

Las mediciones por los métodos calicatas y topográfico se realizaron durante el período comprendido entre Enero y Julio del 2006, logrando conformar una serie histórica de un período de seis meses, extrapolando linealmente los valores para un año.

Finalmente para ajustar los parámetros del modelo de Perdida de Material se utilizó la serie histórica de datos medidos por el método topográfico.

\subsubsection{Medición Características Geométricas}

Durante los años 2003 y 2004 vialidad regional llevó adelante un programa que consistió en georreferenciar los caminos pavimentados y no pavimentados de la región de Antofagasta, para incorporarlos a su base de datos GIS.

\subsubsection{Almacenamiento de Datos}

Los datos tomados en el área de estudio fueron almacenados en la base de datos de la Dirección Nacional de Vialidad, en formularios y resumidos en cuadros que se presentan en el próximo capítulo. 


\section{CAPITULO IV}

\section{ANALISIS DE RESULTADOS}

\subsection{INTRODUCCION}

Las relaciones del HDM-III que predicen el deterioro de los caminos no pavimentados y los efectos del mantenimiento, se han mantenido en el HDM-4. En el HDM-III las unidades de rugosidad se especificaron en términos del "Índice Cuarto Carro", QI. Considerando que la rugosidad en el HDM-4 se especifica como IRI en $\mathrm{m} / \mathrm{km}$, es que la nueva versión del HDM ha reformulado las relaciones de los modelos de deterioro declaradas por Paterson ${ }^{1} \mathrm{y}$ Watanatada ${ }^{2}$ para reflejar la rugosidad en unidades de IRI.

A causa de la alta variabilidad de las propiedades de los materiales, drenaje, erosión de la superficie y los altos niveles de rugosidad de los caminos no pavimentados, los errores de la predicción tienden a ser grandes, el error estándar del estudio brasileño es del orden de 1,5 a 2,5 m/km IRI, para un nivel del confianza del $95 \%$.

El modelo adoptado por el HDM circunscribe a la rugosidad a un límite superior, o rugosidad máxima $\left(R I_{\max }\right)$, desde la cual una función convexa decreciente con tasa exponencial inversa desciende hasta el valor esperado.

En el estudio de Brasil, la rugosidad máxima $\left(R I_{\max }\right)$ se fundamentó en función de las propiedades de los materiales y la geometría del camino, mientras que la proporción de progresión de rugosidad está en función de la rugosidad en un tiempo determinado, la rugosidad máxima, el tiempo, el tránsito (vehículos livianos y pesados), las propiedades de los materiales y el clima.

Se determinó, además, que la magnitud de reducción de la rugosidad depende de la rugosidad antes de las actividades de mejoramiento, de las propiedades

\footnotetext{
${ }^{1}$ William Paterson D.O. (1987). "Road Deterioration and Maintenance Effects: Models for Planning and Management. Highway Desing and Maintenance Standards Series", World Bank Transportation department, Washington D.C.

${ }^{2}$ Watanatada et al. (1987), The Highway Design and Maintenance Standards Model, Volume 1

- Description, The World Bank, John Hopkins University Press.
} 
de los materiales y de la rugosidad mínima $\left(R I_{\min }\right)$.

El modelo del efecto de Reperfilado, nace de la condición de rugosidad más baja que puede alcanzar el camino $\left(R I_{\text {mín }}\right)$ y sobre la cual las actividades de mantenimiento no tienen mayor efecto. La relación para predecir la rugosidad después del reperfilado se expresa como una función lineal de la rugosidad antes de las actividades de mantenimiento, de la relación de polvo y la rugosidad mínima.

Para el caso del presente estudio, las vías consideradas son aquellas que están insertas en el programa del Ministerio de Obras Públicas "Caminos Básicos 5.000", rutas que no consultas estrategias de conservación. Por tanto, se consideró que la rugosidad máxima es aquella alcanzada por el camino luego de transcurrido tres o más años desde la puesta en operación y sin ningún tipo de mantenimiento.

El HDM-4 fue desarrollado como parte del "International Study of Highway Development and Management Tools" (ISOHDM), proyecto internacional para desarrollar nuevos mecanismos de análisis de inversión vial, que contó con el patrocinio del Banco Mundial, la AIPCR, el Departamento de Desarrollo Internacional del Reino Unido, El Banco de Desarrollo Asiático y la Administración Nacional de Caminos Sueca entre otros. La Dirección Nacional de Vialidad de Chile adopto el HDM-4, para la asignación de los recursos de la red vial, permitiendo evaluar económicamente los proyectos viales y asignar recursos más eficientemente.

Las variables explicativas de las condiciones consideradas en los modelos de deterioro de los caminos no pavimentados (grado de curvatura en planta de los caminos, pendiente de la vías, relación de gradación de polvo del material granular, precipitación media mensual de la zona, etc.), desde el punto de vista ingenieril, son las más incidentes en las variables respuestas (rugosidad máxima, mínima y progresión de la misma). No obstante, la variable pluviométrica, sobre todo en la extensa aridez de la macro zona norte de Chile, sería la menos atingente o con menor poder explicativo, debido a que es casi 
nula en toda época del año, lo que podría hacer aconsejable su exclusión del modelo.

Por otro lado, es valido pensar que las características geográficas y climáticas de la zona en que se emplazan los caminos de la región de Antofagasta, sugieran otras variables a considerar en el modelo. Por ejemplo, el gradiente térmico (temperaturas bajo cero por la noche y sobre 35 grados Celsius por el día) y/o la cohesión. La temperatura incide en la humedad relativa del ambiente, la que a su vez incide en la humedad natural de las carpetas granulares. Al disminuir la humedad del material granular también disminuye su cohesión y por tanto se incrementa el desprendimiento de material fino de las carpetas y con ello acrecienta el efecto "calamina" (ondulación transversal al eje del camino de la superficie de rodado).

Otra variable que el modelo de Paterson ${ }^{3}$ y Watanatada ${ }^{4}$ no consulta es el peralte, el cual controla y disminuye el efecto de la fuerza centrífuga en las curvas circulares de pequeño radio, como es el caso de los caminos por laderas de cerros (caminos cornisas).

En definitiva, ante la ausencia de lluvias y el alto gradiente térmico en la región de Antofagasta, lo recomendable desde el punto de vista de la ingeniería vial, sería eliminar la variable pluviométrica del modelo e incorporar la variable cohesión.

Ante la incorporación y/o eliminación de variables del modelo, estaríamos desarrollando un nuevo modelo de deterioro, y lo que se ha planteado en el presente estudio es ajustar los parámetros lineales de los modelos en cuestión.

Las variables, que podrían mejorar el ajuste del modelo a las condiciones de la región de Antofagasta, no han sido incorporadas en primer lugar, por que en el estudio se plantea ajustar los parámetros lineales de los modelos de deterioro

\footnotetext{
${ }^{3}$ William Paterson D.O. (1987). "Road Deterioration and Maintenance Effects: Models for Planning and Management. Highway Desing and Maintenance Standards Series", World Bank Transportation department, Washington D.C.

${ }^{4}$ Watanatada et al. (1987), The Highway Design and Maintenance Standards Model, Volume 1

- Description, The World Bank, John Hopkins University Press.
} 
definidos en el HDM-4, y en segundo lugar, debido a que el modelo original no consulta dichas variables, la Dirección de Vialidad de Chile no ha considerado evaluarlas en el tiempo. Situación que el autor ha informado a la Dirección de Vialidad, lo relevante que en el futuro se realicen mediciones y pueda ser evaluada su incidencia en los modelos de deterioro y comportamiento de los caminos no pavimentados.

Por tanto, dada la falta de datos, desde el punto de vista ingenieril, no se ha considerado incorporar otras variables que pudiesen influir en las condiciones de los caminos no pavimentados en la Región de Antofagasta.

No obstante, en el contexto del presente estudio, se buscar ajustar los diferentes modelos de deterioro, estimando los parámetros asociados a las variables explicatorias de los modelos de Paterson para Brasil, en función de las características físicas geométricas y naturales del entorno de las vías no pavimentadas de la región de Antofagasta, Chile.

La Dirección de Vialidad de Chile ha desarrollado un sistema de medición que permita ajustar el HDM-4 para todo el país. Sin embargo la heterogeneidad de la geografía hace aconsejable realizar mediciones adicionales que capturen los efectos de la diversidad de climas presentes en el territorio nacional sobre los caminos no pavimentados.

Respecto de la base de datos con que se trabajó para determinar los ajustes a las variables independientes de los modelos de deterioro del HDM-4, estos se obtienen de datos históricos de la Dirección Nacional de Vialidad de Chile, de la Dirección de Meteorología de Chile, Mapas Isoyetas de WMO (Organización Mundial de Meteorología) y de los datos levantado en campo, en el marco del presente estudio.

Referente al levantamiento de datos, es importante dejar presente, que estos se realizaron bajo estándares metodológicos establecidos y aceptados por los estamentos internacionales de medición. Las mediciones pluviométricas son realizadas por centros de medición de superficie que pertenecen a la red nacional de meteorología. Los muestreos y ensayos de laboratorio de 
materiales son realizados por el laboratorio regional de Vialidad, según los estándares establecidos en las Normas Chilenas de Ensayos o Normas Internacionales de Ensayos de Materiales (ASTM). Las mediciones de tránsito medio anual de vehículos y las mediciones de rugosidad (IRI - índice de rugosidad internacional) son realizadas por la unidad de Gestión Vial del Ministerio de Obras Públicas. Lo expuesto garantiza la homogeneidad en la recolección de la información.

Por tanto, todos los datos (bases históricas y de mediciones recientes) componen el "Marco Estadístico" con que se trabajo en los ajustes de los parámetros lineales que gobiernan las variables independientes, mediante modelos de regresión lineal múltiple.

Los datos que conforman el marco estadístico se encuentran tabulados (ordenados y detallados) a lo largo del desarrollo del presente capítulo, en cada uno de los apartados en que se analiza y ajusta alguno de los modelos de deterioro.

En resumen, en atención a lo expuesto, en el presente capítulo se estiman los parámetros asociados a las variables independientes de los modelos originales de deterioro, formulados en los estudios del HDM-4, de tal forma, de obtener los nuevos parámetros que definan la incidencia de las variables independiente en los modelos de deterioro, para las condiciones particulares de la región de Antofagasta, Chile.

\subsection{CARACTERISTICAS MEDIDAS EN LOS TRAMOS}

\subsubsection{Características Geométricas}

Las características geométricas de los 21 tramos seleccionados para este estudio se resumen en la tabla 4.1. No obstante, las variables curvaturas y subidas/bajadas señaladas en la tabla 4.1 denotan bastante heterogeneidad, se ha preferido ajustar el modelo bajo esta condición de diversidad; primero por ser la condición topográfica de las vías no pavimentadas de la región de Antofagasta y en segundo lugar con la finalidad de ajustar el modelo para una 
condición general.

Tabla 4.1: Características geométricas de los tramos

\begin{tabular}{|c|c|c|c|c|c|}
\hline \multirow[t]{2}{*}{ TRAMO } & LARGO & ANCHO & $\begin{array}{c}\text { CURVA } \\
\text { Horizontal }\end{array}$ & $\begin{array}{l}\text { SUBIDAS + } \\
\text { BAJADAS }\end{array}$ & ALTURA \\
\hline & (m) & (m) & $(\% / k m)$ & $(\mathrm{m} / \mathrm{km})$ & (msnm) \\
\hline CR1 & 1.500 & 7 & 28,0 & 17,00 & 3.000 \\
\hline CR2 & 1.500 & 7 & 92,7 & 17,00 & 3.030 \\
\hline CR3 & 3.000 & 6 & 8,0 & 5,00 & 1.150 \\
\hline CR4 & 3.000 & 6 & 10,7 & 5,00 & 950 \\
\hline CR5 & 3.000 & 8 & 0,0 & 8,00 & 2.700 \\
\hline CR6 & 3.000 & 8 & 0,0 & 8,00 & 2.800 \\
\hline CR7 & 2.000 & 8 & 3,0 & 20,00 & 2.700 \\
\hline CR8 & 2.000 & 8 & 9,0 & 20,00 & 2.800 \\
\hline CR9 & 2.000 & 8 & 3,0 & 20,00 & 2.900 \\
\hline CR10 & 3.000 & 7 & 47,7 & 5,00 & 2.500 \\
\hline CR11 & 3.000 & 7 & 7,7 & 5,00 & 2.570 \\
\hline CR12 & 3.000 & 7 & 5,0 & 5,00 & 1.300 \\
\hline CR13 & 3.000 & 7 & 13,0 & 5,00 & 1.400 \\
\hline CR14 & 2.500 & 9 & 128,8 & 5,00 & 3.150 \\
\hline CR15 & 2.500 & 9 & 69,2 & 5,00 & 3.200 \\
\hline CR16 & 4.000 & 7 & 13,0 & 33,00 & 500 \\
\hline CR17 & 4.000 & 7 & 65,8 & 25,00 & 400 \\
\hline CR18 & 4.000 & 7 & 54,5 & 22,00 & 320 \\
\hline CR19 & 4.000 & 7 & 2,0 & 20,00 & 250 \\
\hline CR20 & 3.000 & 6 & 0,0 & 9,00 & 1.300 \\
\hline CR21 & 3.000 & 6 & 11,3 & 9,00 & 1.600 \\
\hline Media & 2.857 & 7 & 27 & 13 & 1.930 \\
\hline Desviación Est. & 760,64 & 0,89 & 35,74 & 8,50 & 1057,92 \\
\hline Mínimo & 1.500 & 6 & 0 & 5 & 250 \\
\hline Máximo & 4.000 & 9 & 129 & 33 & 3.200 \\
\hline
\end{tabular}

La geometría del camino queda definida por las siguientes variables:

- Subidas del camino (RF): Promedio de las pendientes en el tramo de análisis, se expresa en metros por kilómetros y su medición se realiza según se indica en el esquema de la figura 3.6 del capítulo tres.

- Curvatura horizontal (HC): Promedio de grado de curvatura en el tramo de análisis, se expresa en grados sexagesimales por kilómetro y se cuantifica según se señala en la figura 3.6 del capítulo tres. 
- Ancho del camino: Distancia entre los bordes del camino, se expresa en metros.

- Espesor de la capa de grava: Corresponde al espesor remanente que presenta la capa de material granular en el tramo en estudio, se expresa en milímetros.

\subsubsection{Características Geotécnicas}

El Tipo de Suelo se caracteriza principalmente con las siguientes variables:

- Tamaño Máximo: Es el tamaño de los áridos, definidos como la abertura de la malla ASTM equivalente, a través de la cual pasa el 95\% del material en peso y se mide en milímetros.

- Material Que Pasa Tamiz: Es la cantidad de material granular que pasa la malla ASTM expresado en porcentajes de la masa:

- $\quad \mathrm{ASTM} \mathrm{N}^{\circ} 10=$ abertura de $2,0 \mathrm{~mm}$

- $\quad$ ASTM N $\mathrm{N}^{\circ} 40=$ abertura de 0,425 mm

- $\quad \mathrm{ASTM} \mathrm{N}^{\circ} 200=$ abertura de $0,075 \mathrm{rnm}$

- Índice De Plasticidad (IP): Es la capacidad de absorción de humedad de un material, sin que .pierda su consistencia plástica, definida según los límites de Atterberg entre el límite líquido (LL) y el límite plástico (LP) expresado en porcentaje.

- Limite Líquido (LL): Es el contenido de humedad, en que el suelo pasa de consistencia plástica a líquida, expresado en el \% y según el ensayo normal del LL.

- Limite Plástico (LP): Es el contenido de humedad, en que el suelo deja de tener consistencia plástica, según el ensayo normal del LP y expresado en \%.

Los ensayos para determinar las características del suelo de la carpeta de 
rodadura fueron realizados y entregados por Laboratorio Nacional de Vialidad y por el Laboratorio Regional Metropolitano.

El estudio sólo se centró en los caminos no pavimentados de la Región de Antofagasta incluidos en el programa de gobierno "Caminos Básicos 5.000"; que corresponden a aquellos caminos con capas de rodado de gravas estabilizadas con bischofita.

En la tabla 4.2 se resume las características geotécnicas de los tramos y en el anexo fotos se puede observar la textura de los suelos.

Tabla 4.2: Características geotécnicas de los tramos

\begin{tabular}{|c|c|c|c|c|c|c|c|c|c|}
\hline \multirow{2}{*}{ TRAMO } & \multirow{2}{*}{$\begin{array}{c}\text { ESPESOR } \\
\text { CARPETA } \\
\mathrm{mm}\end{array}$} & \multirow{2}{*}{$\begin{array}{c}\text { TAMAÑO } \\
\text { MÁXIMO } \\
\text { mm }\end{array}$} & \multicolumn{3}{|c|}{$\%$ PASA MALLA } & \multirow{2}{*}{$\begin{array}{l}\text { LL } \\
\%\end{array}$} & \multirow{2}{*}{$\begin{array}{l}\text { IP } \\
\%\end{array}$} & \multirow{2}{*}{$\begin{array}{c}\text { DENSIDAD } \\
\text { TOTAL } \\
\mathrm{kg} / \mathrm{m} 3 \\
\end{array}$} & \multirow{2}{*}{$\begin{array}{c}\text { CBR } \\
\%\end{array}$} \\
\hline & & & 10 & 40 & 200 & & & & \\
\hline CR1 & 120 & 40 & 50 & 33 & 17 & 22,5 & 5 & 2.076 & 100 \\
\hline CR2 & 120 & 40 & 53 & 36 & 17 & 23,7 & 4,8 & 2.101 & 100 \\
\hline CR3 & 150 & 40 & 55 & 34 & 22 & 35 & 6 & 2.153 & 80 \\
\hline CR4 & 150 & 40 & 55 & 34 & 22 & 35 & 6 & 2.153 & 80 \\
\hline CR5 & 120 & 40 & 53 & 36 & 14 & 23 & 4 & 2.088 & 98 \\
\hline CR6 & 120 & 40 & 55 & 38 & 14 & 24 & 4 & 2.035 & 100 \\
\hline CR7 & 150 & 40 & 46 & 26 & 8 & IND & N.P. & 2.249 & 84 \\
\hline CR8 & 150 & 40 & 46 & 26 & 8 & IND & N.P. & 2.249 & 84 \\
\hline CR9 & 150 & 40 & 46 & 26 & 8 & IND & N.P. & 2.249 & 84 \\
\hline CR10 & 120 & 25 & 47 & 28 & 17 & 25 & 9 & 2.138 & 88 \\
\hline CR11 & 120 & 25 & 49 & 30 & 18 & 28 & 10 & 2.145 & 92 \\
\hline CR12 & 200 & 40 & 50 & 35 & 20 & 20 & 1,5 & 2.020 & 80 \\
\hline CR13 & 200 & 40 & 69 & 44 & 30 & 30 & 2 & 2.105 & 92 \\
\hline CR14 & 150 & 40 & 55 & 35 & 21 & 20,5 & 6,8 & 2.188 & 85 \\
\hline CR15 & 150 & 40 & 55 & 35 & 22 & 21 & 8 & 2.185 & 79 \\
\hline CR16 & 200 & 40 & 41 & 22 & 8 & 17 & 2 & 2.230 & 100 \\
\hline CR17 & 200 & 40 & 41 & 22 & 8 & 17 & 2 & 2.230 & 100 \\
\hline CR18 & 200 & 40 & 46 & 27 & 8 & 19 & 2 & 2.281 & 100 \\
\hline CR19 & 200 & 40 & 46 & 27 & 8 & 19 & 2 & 2.281 & 100 \\
\hline CR20 & 150 & 40 & 40 & 30 & 12 & 25 & N.P. & 2.219 & 100 \\
\hline CR21 & 150 & 40 & 44 & 30 & 16 & 24 & N.P. & 2.254 & 100 \\
\hline Media & 155,7 & 38,6 & 49,6 & 31,1 & 15,1 & 23,8 & 4,7 & 2172,8 & 91,7 \\
\hline Desv. Estándar & 31,4 & 4,5 & 6,7 & 5,6 & 6,4 & 5,3 & 2,7 & 80,0 & 8,6 \\
\hline Mínimo & 120,0 & 25,0 & 40,0 & 22,0 & 8,0 & 17,0 & 1,5 & 2020,0 & 79,0 \\
\hline Máximo & 200,0 & 40,0 & 69,0 & 44,0 & 30,0 & 35,0 & 10,0 & 2281,0 & 100,0 \\
\hline
\end{tabular}




\subsubsection{Tránsito solicitante}

La forma de medir el tránsito de estos caminos se efectuó basándose en los estudios del Plan Nacional de Censo de los años 2002, 2004 y 2006 realizados por la Dirección de Vialidad, dicho plan, sólo se realiza en años pares.

En la tabla 4.3 se sintetizan los tránsitos consultados para los tramos seleccionados.

Tabla 4.3: Tránsito de los tramos en estudio - Año 2006 (veh/día)

\begin{tabular}{|c|c|c|c|c|c|c|}
\hline \multirow{2}{*}{ TRAMO } & \multirow{2}{*}{ AUTO } & \multirow{2}{*}{ CAMIONETA } & CAMION & CAMION & \multirow{2}{*}{ BUS } & \multirow{2}{*}{ TOTAL } \\
\cline { 4 - 5 } & & & 2 EJES & MÁS 2 EJES & & \\
\hline CR1 & 1 & 31 & 9 & 22 & 3 & 66 \\
\hline CR2 & 1 & 31 & 9 & 22 & 3 & 66 \\
\hline CR3 & 12 & 34 & 16 & 62 & 2 & 126 \\
\hline CR4 & 12 & 34 & 16 & 62 & 2 & 126 \\
\hline CR5 & 1 & 13 & 1 & 1 & 1 & 17 \\
\hline CR6 & 1 & 13 & 1 & 1 & 1 & 17 \\
\hline CR7 & 12 & 13 & 2 & 1 & 1 & 29 \\
\hline CR8 & 10 & 12 & 4 & 2 & 2 & 30 \\
\hline CR9 & 10 & 12 & 4 & 2 & 2 & 30 \\
\hline CR10 & 1 & 18 & 1 & 1 & 1 & 22 \\
\hline CR11 & 1 & 18 & 1 & 1 & 1 & 22 \\
\hline CR12 & 102 & 124 & 24 & 34 & 35 & 319 \\
\hline CR13 & 102 & 124 & 24 & 34 & 35 & 319 \\
\hline CR14 & 7 & 10 & 1 & 0 & 2 & 20 \\
\hline CR15 & 7 & 10 & 1 & 0 & 2 & 20 \\
\hline CR16 & 12 & 46 & 21 & 108 & 3 & 190 \\
\hline CR17 & 12 & 46 & 21 & 108 & 3 & 190 \\
\hline CR18 & 12 & 46 & 21 & 108 & 3 & 190 \\
\hline CR19 & 12 & 46 & 21 & 108 & 3 & 190 \\
\hline CR20 & 1 & 10 & 6 & 43 & 2 & 62 \\
\hline CR21 & 1 & 10 & 6 & 43 & 2 & 62 \\
\hline Tasa/Anual & $5,50 \%$ & $6,00 \%$ & $5,00 \%$ & $4,00 \%$ & $4,50 \%$ & $5,00 \%$ \\
\hline Tasa/mensual & $0,46 \%$ & $0,50 \%$ & $0,42 \%$ & $0,33 \%$ & $0,38 \%$ & $0,42 \%$ \\
\hline Total & 330 & 701 & 210 & 763 & 109 & 2.113 \\
\hline Media & 16 & 33 & 10 & 36 & 5 & 101 \\
\hline Desv. ST. & 29,10 & 33,09 & 8,96 & 41,00 & 9,94 & 97,07 \\
\hline Mínim0 & 1 & 10 & 1 & 0 & 1 & 17 \\
\hline Máxim0 & 102 & 124 & 24 & 108 & 35 & 319 \\
\hline & & & & & & \\
\hline
\end{tabular}




\subsection{ANALISIS PLUVIOMETRICO EN EL AREA DE ESTUDIO}

En virtud del análisis estadístico de la lluvia caída durante el período de la experiencia, enero del año 2005 a agosto del año 2006, de la ubicación geográfica de la zona de estudio - Desierto de Atacama - y al no contar con registros de IRI durante los exiguos períodos de lluvias, se determinó, en lo fundamental, que al contar con registros pluviométricos tan bajos no existe la posibilidad de hacer una diferenciación entre zona seca y zona húmeda, por ello se ha considerado los 12 meses del año como estación seca.

Tabla 4.4: Precipitación año 2005 del norte grande

\begin{tabular}{|c|c|c|c|c|c|c|c|c|c|c|c|c|c|c|}
\hline \multirow{2}{*}{ ESTACIONES } & \multicolumn{12}{|c|}{ PRECIPITACION MEDIA MENSUAL EN MM - 2005} & \multirow{2}{*}{$\begin{array}{c}\text { Total } \\
\text { año } \\
(\mathrm{mm}) \\
\end{array}$} & \multirow{2}{*}{$\begin{array}{l}\text { Prom/mes } \\
(\mathrm{mm})\end{array}$} \\
\hline & Ene & Feb & Mar & Abr & May & Jun & Jul & Ago & Sep & Oct & Nov & Dic & & \\
\hline Central Chapiquiña & 26,0 & 68,5 & 2,0 & 0,0 & 0,0 & 0,0 & 0,0 & 0,0 & 7,5 & 0,0 & 0,0 & 22,3 & 126,3 & 10,53 \\
\hline Ollagüe & 31,8 & 29,6 & 5,3 & 0,0 & 0,0 & 0,0 & 0,0 & 0,3 & 0,5 & 1,8 & 2,4 & 3,9 & 75,6 & 6,3 \\
\hline Embalse Conchi & 18,5 & 3,5 & 1,0 & 0,0 & 0,0 & 0,0 & 0,0 & 0,0 & 5,5 & 0,0 & 0,0 & 0,0 & 28,5 & 2,38 \\
\hline Calama & 0,0 & 0,5 & 0,0 & 3,5 & 2,5 & 0,0 & 0,0 & 0,0 & 0,0 & 0,0 & 0,0 & 0,0 & 6,5 & 0,54 \\
\hline Antofagasta & 0,0 & 0,0 & 0,0 & 0,5 & 0,0 & 0,0 & 0,0 & 0,0 & 0,0 & 0,0 & 0,0 & 0,0 & 0,5 & 0,04 \\
\hline Copiapó & 0,0 & 0,0 & 0,0 & 1,8 & 0,0 & 0,0 & 10,2 & 6,7 & 0,0 & 0,0 & 0,0 & 0,0 & 18,7 & 1,56 \\
\hline Embalse Lautaro & 0,0 & 0,0 & 0,0 & 15,5 & 0,0 & 0,0 & 10,0 & 4,0 & 2,0 & 0,0 & 0,0 & 0,0 & 31,5 & 2,63 \\
\hline Vallenar & 0,0 & 0,0 & 0,0 & 0,0 & 1,0 & 0,0 & 7,5 & 20,1 & 0,0 & 0,0 & 0,0 & 0,0 & 28,6 & 2,38 \\
\hline Media & 9,5 & 12,8 & 1,0 & 2,7 & 0,4 & 0,0 & 3,5 & 3,9 & 1,9 & 0,2 & 0,3 & 3,3 & 39,5 & 3,3 \\
\hline Desv. Estándar & 13,6 & 24,7 & 1,9 & 5,3 & 0,9 & 0,0 & 4,8 & 7,0 & 2,9 & 0,6 & 0,8 & 7,8 & 41,7 & 3,5 \\
\hline Mínima & 31,8 & 68,5 & 5,3 & 15,5 & 2,5 & 0,0 & 10,2 & 20,1 & 7,5 & 1,8 & 2,4 & 22,3 & 126,3 & 10,5 \\
\hline Máxima & 0,0 & 0,0 & 0,0 & 0,0 & 0,0 & 0,0 & 0,0 & 0,0 & 0,0 & 0,0 & 0,0 & 0,0 & 0,5 & 0,0 \\
\hline
\end{tabular}

Tabla 4.5: Precipitación año 2006 del norte grande

\begin{tabular}{|c|c|c|c|c|c|c|c|c|c|}
\hline \multirow{2}{*}{ ESTACIONES } & \multicolumn{7}{|c|}{ PRECIPITACION MEDIA MENSUAL EN MM - 2006} & \multirow{2}{*}{$\begin{array}{l}\text { Total } \\
\text { año }\end{array}$} & \multirow{2}{*}{ Prom/mes } \\
\hline & Ene & Feb & Mar & Abr & May & Jun & Jul & & \\
\hline Central Chapiquiña & 26,5 & 94,5 & 34,5 & 0,0 & 0,0 & 0,0 & 0,0 & 155,5 & 22,2 \\
\hline Ollagüe & 31,8 & 29,6 & 5,3 & 0,0 & 0,0 & 0,0 & 0,0 & 66,7 & 9,5 \\
\hline Embalse Conchi & 9,0 & 24,2 & 0,0 & 0,0 & 0,0 & 0,0 & 0,0 & 33,2 & 4,7 \\
\hline Calama & 0,2 & 0,5 & 0,0 & 0,0 & 0,0 & 0,0 & 0,0 & 0,7 & 0,1 \\
\hline Antofagasta & 0,0 & 0,0 & 0,0 & 0,0 & 0,0 & 0,0 & 0,0 & 0,0 & 0,0 \\
\hline Copiapó & 0,0 & 0,0 & 0,0 & 0,0 & 0,0 & 0,0 & 0,0 & 0,0 & 0,0 \\
\hline Embalse Lautaro & 0,0 & 0,0 & 0,0 & 0,0 & 1,0 & 0,0 & 0,0 & 1,0 & 0,1 \\
\hline Vallenar & 0,0 & 0,0 & 0,0 & 0,0 & 0,0 & 1,0 & 1,0 & 2,0 & 0,3 \\
\hline Media & 8,4 & 18,6 & 5,0 & 0,0 & 0,1 & 0,1 & 0,1 & 32,4 & 4,6 \\
\hline Desviación Estándar & 13,2 & 33,0 & 12,1 & 0,0 & 0,4 & 0,4 & 0,4 & 55,2 & 7,9 \\
\hline Mínima & 31,8 & 94,5 & 34,5 & 0,0 & 1,0 & 1,0 & 1,0 & 155,5 & 22,2 \\
\hline Máxima & 0,0 & 0,0 & 0,0 & 0,0 & 0,0 & 0,0 & 0,0 & 0,0 & 0,0 \\
\hline
\end{tabular}


Tabla 4.6: Precipitaciones totales y promedios de la zona de estudio

\begin{tabular}{|l|c|c|c|c|c|}
\hline \multirow{2}{*}{ ESTACIONES } & \multicolumn{5}{|c|}{ Pluviometría Enero 2005 a Agosto 2006 (mm) } \\
\cline { 2 - 6 } & $\begin{array}{c}\text { Total } \\
\mathbf{1 9} \text { meses }\end{array}$ & $\begin{array}{c}\text { Prom } \\
\mathbf{1 2} \text { meses }\end{array}$ & $\begin{array}{c}\text { Prom/mes } \\
\text { Ene_05 - Ago_06 }\end{array}$ & $\begin{array}{c}\text { Prom/año } \\
\text { normal }\end{array}$ & $\begin{array}{c}\text { Prom/mes } \\
\text { normal }\end{array}$ \\
\hline Ollagüe & 142,30 & 89,87 & 7,49 & 40,80 & 3,40 \\
\hline Embalse Conchi & 61,70 & 38,97 & 3,25 & 17,20 & 1,43 \\
\hline Calama & 7,20 & 4,55 & 0,38 & 5,70 & 0,48 \\
\hline Antofagasta & 0,50 & 0,32 & 0,03 & 1,70 & 0,14 \\
\hline Media & 23,1 & 14,6 & 1,2 & 8,2 & 0,7 \\
\hline Desviación Estándar & 33,6 & 21,2 & 1,8 & 8,0 & 0,7 \\
\hline Mínima & 61,7 & 39,0 & 3,2 & 17,2 & 1,4 \\
\hline Máxima & 0,5 & 0,3 & 0,0 & 1,7 & 0,1 \\
\hline
\end{tabular}

En las tablas $4.4,4.5$ y 4.6 se entregan los valores de precipitación mensual promedio, medidos en las Estaciones Pluviométricas descritas en el Capítulo tres y que se han considerado en el presente estudio.

\subsection{DATOS DE RUGOSIDAD EN LOS TRAMOS TESTIGOS}

En las tablas siguientes se muestran los datos de rugosidad obtenidos de la base de datos de la Dirección Regional de Vialidad y aquellos medidos en el área de estudio durante el período de observación.

Las primeras mediciones realizadas por el Laboratorio Nacional de Vialidad datan del mes de octubre del año 2003 y las últimas son las que se llevaron a efecto durante el presente estudio en el mes enero del año 2006.

En la medición de la rugosidad de los caminos, según lo detallado en el capítulo tres, se utilizó el rugosímetro MIS4, el que permitió la recolección y presentación de la regularidad del pavimento o IRI. Los valores que conforman la base del marco Estadístico, fueron procesaron y tabulados en las tablas 4.7, $4.8,4.9,4.10,4.11,4.12,4.13,4.14,4.15,4.16$ y 4.17.

Recordemos que el principio de funcionamiento está basado directamente en la evaluación de la respuesta dinámica del vehículo, lo que permite definir al rugosímetro MIS4 de clase 3. 
Tabla 4.7: Rugosidad medida en tramo CR1

\begin{tabular}{|c|c|c|c|c|c|}
\hline \multicolumn{7}{|c|}{ IRI (m/km) Tramo CR1 - Ruta 21 - Ch } \\
\hline \multicolumn{2}{|c|}{ Kilómetro } & Oct-03 & Abr-04 & Nov-04 & Ene-06 \\
\hline Inicial & Final & (m/km) & (m/km) & (m/km) & (m/km) \\
\hline 70,00 & 70,10 & 3,6 & 3,0 & 2,2 & 8,4 \\
\hline 70,10 & 70,20 & 2,3 & 2,1 & 2,6 & 5,3 \\
\hline 70,20 & 70,30 & 3,3 & 2,8 & 2,3 & 4,9 \\
\hline 70,30 & 70,40 & 2,9 & 2,1 & 2,2 & 4,6 \\
\hline 70,40 & 70,50 & 3,4 & 3,2 & 2,6 & 4,6 \\
\hline 70,50 & 70,60 & 3,0 & 2,7 & 2,5 & 3,9 \\
\hline 70,60 & 70,70 & 3,3 & 2,8 & 2,2 & 5,5 \\
\hline 70,70 & 70,80 & 2,3 & 3,0 & 3,5 & 4,0 \\
\hline 70,80 & 70,90 & 2,5 & 2,7 & 2,7 & 3,9 \\
\hline 70,90 & 71,00 & 2,3 & 4,3 & 3,6 & 4,8 \\
\hline 71,00 & 71,10 & 3,3 & 3,6 & 3,0 & 4,6 \\
\hline 71,10 & 71,20 & 2,3 & 4,1 & 5,0 & 10,5 \\
\hline 71,20 & 71,30 & 3,1 & 4,3 & 8,9 & 11,5 \\
\hline 71,30 & 71,40 & 3,0 & 6,8 & 9,1 & 12,1 \\
\hline 71,40 & 71,50 & 2,7 & 7,3 & 8,5 & 12,0 \\
\hline Media & & 2,9 & 3,7 & 4,1 & 6,7 \\
\hline Desviación Estándar & & 0,45 & 1,54 & 2,57 & 3,22 \\
\hline Máximo & & 3,6 & 7,3 & 9,1 & 12,1 \\
\hline Mínimo & & 2,3 & 2,1 & 2,2 & 3,9 \\
\hline
\end{tabular}

Tabla 4.8: Rugosidad medida en tramo CR2

\begin{tabular}{|c|c|c|c|c|c|}
\hline \multicolumn{7}{|c|}{ IRI (m/km) Tramo CR2 - Ruta 21 - Ch } \\
\hline \multicolumn{2}{|c|}{ Kilómetro } & Oct-03 & Abr-04 & Nov-04 & Ene-06 \\
\hline Inicial & Final & (m/km) & (m/km) & (m/km) & (m/km) \\
\hline 71,50 & 71,60 & 3,6 & 4,3 & 3,5 & 6,0 \\
\hline 71,60 & 71,70 & 2,6 & 2,8 & 3,5 & 6,2 \\
\hline 71,70 & 71,80 & 2,6 & 2,6 & 2,9 & 5,1 \\
\hline 71,80 & 71,90 & 2,9 & 2,8 & 3,5 & 5,8 \\
\hline 71,90 & 72,00 & 3,2 & 4,0 & 3,9 & 7,0 \\
\hline 72,00 & 72,10 & 2,9 & 2,9 & 3,9 & 6,5 \\
\hline 72,10 & 72,20 & 6,0 & 5,0 & 9,7 & 8,1 \\
\hline 72,20 & 72,30 & 3,4 & 6,5 & 9,0 & 13,2 \\
\hline 72,30 & 72,40 & 2,7 & 7,8 & 10,1 & 9,1 \\
\hline 72,40 & 72,50 & 3,2 & 5,0 & 5,7 & 9,8 \\
\hline 72,50 & 72,60 & 2,5 & 2,6 & 4,7 & 6,9 \\
\hline 72,60 & 72,70 & 2,6 & 2,6 & 3,2 & 6,6 \\
\hline 72,70 & 72,80 & 2,5 & 2,4 & 2,7 & 7,7 \\
\hline 72,80 & 72,90 & 2,8 & 2,5 & 3,7 & 8,7 \\
\hline 72,90 & 73,00 & 2,7 & 2,7 & 2,5 & 9,5 \\
\hline Media & & 3,1 & 3,8 & 4,8 & 7,7 \\
\hline Desviación Estándar & & 0,88 & 1,66 & 2,59 & 2,07 \\
\hline Máximo & & 6,0 & 7,8 & 10,1 & 13,2 \\
\hline Mínimo & & 2,5 & 2,4 & 2,5 & 5,1 \\
\hline
\end{tabular}


Tabla 4.9: Rugosidad medida en tramo CR3 y CR4

\begin{tabular}{|c|c|c|c|c|c|}
\hline \multicolumn{3}{|c|}{ IRI (m/km) Tramo CR3 - Ruta B-55 } & \multicolumn{3}{|c|}{ IRI (m/km) Tramo CR4 - Ruta B-55 } \\
\hline \multicolumn{2}{|c|}{ Kilómetro } & \multirow{2}{*}{$\begin{array}{l}\text { Ene-06 } \\
(\mathrm{m} / \mathrm{km})\end{array}$} & \multicolumn{2}{|c|}{ Kilómetro } & \multirow{2}{*}{$\begin{array}{l}\text { Ene-06 } \\
(\mathrm{m} / \mathrm{km})\end{array}$} \\
\hline Inicial & Final & & Inicial & Final & \\
\hline 15,60 & 15,70 & 10,1 & 22,00 & 22,10 & 6,2 \\
\hline 15,70 & 15,80 & 9,8 & 22,10 & 22,20 & 8,4 \\
\hline 15,80 & 15,90 & 10,4 & 22,20 & 22,30 & 6,6 \\
\hline 15,90 & 16,00 & 10,3 & 22,30 & 22,40 & 4,8 \\
\hline 16,00 & 16,10 & 10,1 & 22,40 & 22,50 & 6,9 \\
\hline 16,10 & 16,20 & 9,6 & 22,50 & 22,60 & 6,2 \\
\hline 16,20 & 16,30 & 9,4 & 22,60 & 22,70 & 5,5 \\
\hline 16,30 & 16,40 & 8,5 & 22,70 & 22,80 & 5,8 \\
\hline 16,40 & 16,50 & 9,0 & 22,80 & 22,90 & 7,5 \\
\hline 16,50 & 16,60 & 9,3 & 22,90 & 23,00 & 4,6 \\
\hline 16,60 & 16,70 & 6,6 & 23,00 & 23,10 & 5,8 \\
\hline 16,70 & 16,80 & 4,0 & 23,10 & 23,20 & 4,8 \\
\hline 16,80 & 16,90 & 6,5 & 23,20 & 23,30 & 5,5 \\
\hline 16,90 & 17,00 & 7,1 & 23,30 & 23,40 & 5,1 \\
\hline 17,00 & 17,10 & 5,8 & 23,40 & 23,50 & 3,7 \\
\hline 17,10 & 17,20 & 4,0 & 23,50 & 23,60 & 5,0 \\
\hline 17,20 & 17,30 & 8,7 & 23,60 & 23,70 & 3,8 \\
\hline 17,30 & 17,40 & 7,7 & 23,70 & 23,80 & 3,4 \\
\hline 17,40 & 17,50 & 8,4 & 23,80 & 23,90 & 3,0 \\
\hline 17,50 & 17,60 & 8,1 & 23,90 & 24,00 & 3,8 \\
\hline 17,60 & 17,70 & 8,0 & 24,00 & 24,10 & 3,6 \\
\hline 17,70 & 17,80 & 7,5 & 24,10 & 24,20 & 4,0 \\
\hline 17,80 & 17,90 & 6,9 & 24,20 & 24,30 & 3,2 \\
\hline 17,90 & 18,00 & 7,4 & 24,30 & 24,40 & 3,8 \\
\hline 18,00 & 18,10 & 5,7 & 24,40 & 24,50 & 3,9 \\
\hline 18,10 & 18,20 & 7,4 & 24,50 & 24,60 & 4,0 \\
\hline 18,20 & 18,30 & 5,8 & 24,60 & 24,70 & 8,2 \\
\hline 18,30 & 18,40 & 5,0 & 24,70 & 24,80 & 10,2 \\
\hline 18,40 & 18,50 & 8,2 & 24,80 & 24,90 & 10,1 \\
\hline 18,50 & 18,60 & 7,3 & 24,90 & 25,00 & 8,0 \\
\hline \multicolumn{2}{|l|}{ Media } & 7,8 & \multicolumn{2}{|l|}{ Media } & 5,5 \\
\hline \multicolumn{2}{|c|}{ Desviación Estándar } & 1,79 & \multicolumn{2}{|c|}{ Desviación Estándar } & 1,98 \\
\hline \multicolumn{2}{|l|}{ Máximo } & 10,4 & \multicolumn{2}{|l|}{ Máximo } & 10,20 \\
\hline \multicolumn{2}{|l|}{ Mínimo } & 4,0 & \multicolumn{2}{|l|}{ Mínimo } & 3,0 \\
\hline
\end{tabular}


Tabla 4.10: Rugosidad medida en tramo CR5 y CR6

\begin{tabular}{|c|c|c|c|c|c|}
\hline \multicolumn{3}{|c|}{ IRI (m/km) Tramo CR5 - Ruta B-155 } & \multicolumn{3}{|c|}{ IRI (m/km) Tramo CR6 - Ruta B-155 } \\
\hline \multicolumn{2}{|c|}{ Kilómetro } & \multirow{2}{*}{$\begin{array}{l}\text { Ene-06 } \\
(\mathrm{m} / \mathrm{km})\end{array}$} & \multicolumn{2}{|c|}{ Kilómetro } & \multirow{2}{*}{$\begin{array}{l}\text { Ene-06 } \\
(\mathrm{m} / \mathrm{km})\end{array}$} \\
\hline Inicial & Final & & Inicial & Final & \\
\hline 1,00 & 1,10 & 4,1 & 4,50 & 4,60 & 3,9 \\
\hline 1,10 & 1,20 & 2,5 & 4,60 & 4,70 & 4,4 \\
\hline 1,20 & 1,30 & 2,8 & 4,70 & 4,80 & 5,8 \\
\hline 1,30 & 1,40 & 3,8 & 4,80 & 4,90 & 8,2 \\
\hline 1,40 & 1,50 & 3,3 & 4,90 & 5,00 & 5,4 \\
\hline 1,50 & 1,60 & 4,0 & 5,00 & 5,10 & 5,6 \\
\hline 1,60 & 1,70 & 3,4 & 5,10 & 5,20 & 3,8 \\
\hline 1,70 & 1,80 & 3,4 & 5,20 & 5,30 & 3,4 \\
\hline 1,80 & 1,90 & 3,2 & 5,30 & 5,40 & 4,2 \\
\hline 1,90 & 2,00 & 3,4 & 5,40 & 5,50 & 4,2 \\
\hline 2,00 & 2,10 & 4,2 & 5,50 & 5,60 & 3,7 \\
\hline 2,10 & 2,20 & 5,5 & 5,60 & 5,70 & 4,1 \\
\hline 2,20 & 2,30 & 3,4 & 5,70 & 5,80 & 3,2 \\
\hline 2,30 & 2,40 & 4,3 & 5,80 & 5,90 & 3,5 \\
\hline 2,40 & 2,50 & 3,9 & 5,90 & 6,00 & 3,9 \\
\hline 2,50 & 2,60 & 5,3 & 6,00 & 6,10 & 3,1 \\
\hline 2,60 & 2,70 & 3,4 & 6,10 & 6,20 & 3,2 \\
\hline 2,70 & 2,80 & 4,8 & 6,20 & 6,30 & 3,1 \\
\hline 2,80 & 2,90 & 4,0 & 6,30 & 6,40 & 2,9 \\
\hline 2,90 & 3,00 & 4,0 & 6,40 & 6,50 & 2,8 \\
\hline 3,00 & 3,10 & 4,9 & 6,50 & 6,60 & 3,0 \\
\hline 3,10 & 3,20 & 3,6 & 6,60 & 6,70 & 3,5 \\
\hline 3,20 & 3,30 & 3,7 & 6,70 & 6,80 & 3,4 \\
\hline 3,30 & 3,40 & 4,2 & 6,80 & 6,90 & 4,6 \\
\hline 3,40 & 4,00 & 7,9 & 6,90 & 7,00 & 4,9 \\
\hline 3,50 & 3,60 & 4,6 & 7,00 & 7,10 & 3,8 \\
\hline 3,60 & 3,70 & 3,3 & 7,10 & 7,20 & 4,6 \\
\hline 3,70 & 3,80 & 3,1 & 7,20 & 7,30 & 4,7 \\
\hline 3,80 & 3,90 & 3,0 & 7,30 & 7,40 & 4,0 \\
\hline 3,90 & 4,00 & 3,8 & 7,40 & 7,50 & 4,4 \\
\hline \multicolumn{2}{|l|}{ Media } & 3,9 & \multicolumn{2}{|l|}{ Media } & 4,1 \\
\hline \multicolumn{2}{|c|}{ Desviación Estándar } & 1,02 & \multicolumn{2}{|c|}{ Desviación Estándar } & 1,11 \\
\hline \multicolumn{2}{|l|}{ Máximo } & 7,9 & \multicolumn{2}{|l|}{ Máximo } & 8,2 \\
\hline \multicolumn{2}{|l|}{ Mínimo } & 2,5 & \multicolumn{2}{|l|}{ Mínimo } & 2,8 \\
\hline
\end{tabular}


Tabla 4.11: Rugosidad medida en tramo CR7, CR8 y CR9

\begin{tabular}{|c|c|c|c|c|c|c|c|c|c|c|c|}
\hline \multicolumn{4}{|c|}{$\begin{array}{c}\text { IRI (m/km) Tramo CR7 } \\
\text { Ruta B-165 }\end{array}$} & \multicolumn{4}{|c|}{$\begin{array}{c}\text { IRI (m/km) Tramo CR8 } \\
\text { Ruta B-165 }\end{array}$} & \multicolumn{4}{|c|}{$\begin{array}{c}\text { IRI (m/km) Tramo CR9 } \\
\text { Ruta B-165 }\end{array}$} \\
\hline \multicolumn{2}{|c|}{ Kilómetro } & \multirow{2}{*}{\begin{tabular}{|l|} 
Nov-04 \\
$(\mathrm{m} / \mathrm{km})$
\end{tabular}} & \multirow{2}{*}{$\begin{array}{l}\text { Ene-06 } \\
(\mathrm{m} / \mathrm{km})\end{array}$} & \multicolumn{2}{|c|}{ Kilómetro } & \multirow{2}{*}{$\frac{\text { Nov-04 }}{(\mathrm{m} / \mathrm{km})}$} & \multirow{2}{*}{$\begin{array}{l}\text { Ene-06 } \\
(\mathrm{m} / \mathrm{km})\end{array}$} & \multicolumn{2}{|c|}{ Kilómetro } & \multirow{2}{*}{$\begin{array}{l}\text { Nov-04 } \\
(\mathrm{m} / \mathrm{km})\end{array}$} & \multirow{2}{*}{$\frac{\text { Ene-06 }}{(\mathrm{m} / \mathrm{km})}$} \\
\hline Inicial & Final & & & Inicial & Final & & & Inicial & Final & & \\
\hline 37,00 & 37,10 & 2,5 & 3,3 & 39,00 & 39,10 & 3,6 & 4,4 & 41,00 & 41,10 & 4,8 & 5,6 \\
\hline 37,10 & 37,20 & 2,7 & 3,2 & 39,10 & 39,20 & 3,1 & 5,3 & 41,10 & 41,20 & 2,9 & 4,9 \\
\hline 37,20 & 37,30 & 2,3 & 3,1 & 39,20 & 39,30 & 4,4 & 4,2 & 41,20 & 41,30 & 3,8 & 4,3 \\
\hline 37,30 & 37,40 & 2,2 & 3,8 & 39,30 & 39,40 & 4,0 & 6,1 & 41,30 & 41,40 & 2,8 & 4,6 \\
\hline 37,40 & 37,50 & 3,2 & 3,5 & 39,40 & 39,50 & 3,8 & 4,6 & 41,40 & 41,50 & 4,5 & 6,5 \\
\hline 37,50 & 37,60 & 3,6 & 4,6 & 39,50 & 39,60 & 3,1 & 5,0 & 41,50 & 41,60 & 3,0 & 4,7 \\
\hline 37,60 & 37,70 & 3,2 & 4,3 & 39,60 & 39,70 & 3,3 & 5,9 & 41,60 & 41,70 & 3,5 & 4,5 \\
\hline 37,70 & 37,80 & 2,9 & 4,2 & 39,70 & 39,80 & 3,0 & 4,6 & 41,70 & 41,80 & 3,9 & 5,0 \\
\hline 37,80 & 37,90 & 6,6 & 8,1 & 39,80 & 39,90 & 3,0 & 5,4 & 41,80 & 41,90 & 2,8 & 5,9 \\
\hline 37,90 & 38,00 & 2,6 & 5,1 & 39,90 & 40,00 & 3,4 & 5,1 & 41,90 & 42,00 & 7,9 & 9,8 \\
\hline 38,00 & 38,10 & 2,0 & 4,1 & 40,00 & 40,10 & 6,9 & 8,5 & 42,00 & 42,10 & 2,4 & 5,5 \\
\hline 38,10 & 38,20 & 2,5 & 4,5 & 40,10 & 40,20 & 3,4 & 4,1 & 42,10 & 42,20 & 2,4 & 4,5 \\
\hline 38,20 & 38,30 & 3,2 & 5,5 & 40,20 & 40,30 & 3,0 & 4,5 & 42,20 & 42,30 & 3,1 & 6,5 \\
\hline 38,30 & 38,40 & 2,7 & 5,0 & 40,30 & 40,40 & 2,5 & 3,9 & 42,30 & 42,40 & 2,1 & 5,2 \\
\hline 38,40 & 38,50 & 2,9 & 4,6 & 40,40 & 40,50 & 3,5 & 6,6 & 42,40 & 42,50 & 3,1 & 4,3 \\
\hline 38,50 & 38,60 & 3,3 & 3,4 & 40,50 & 40,60 & 3,5 & 4,5 & 42,50 & 42,60 & 3,4 & 5,3 \\
\hline 38,60 & 38,70 & 2,8 & 5,2 & 40,60 & 40,70 & 2,4 & 6,7 & 42,60 & 42,70 & 3,0 & 3,9 \\
\hline 38,70 & 38,80 & 3,3 & 3,8 & 40,70 & 40,80 & 2,3 & 3,6 & 42,70 & 42,80 & 2,8 & 4,6 \\
\hline 38,80 & 38,90 & 2,0 & 3,3 & 40,80 & 40,90 & 2,4 & 4,6 & 42,80 & 42,90 & 3,0 & 4,1 \\
\hline 38,90 & 39,00 & 3,5 & 6,7 & 40,90 & 41,00 & 3,4 & 5,3 & 42,90 & 43,00 & 3,5 & 4,4 \\
\hline \multicolumn{2}{|l|}{ Media } & 3,0 & 4,4 & \multicolumn{2}{|l|}{ Media } & 3,4 & 5,1 & \multicolumn{2}{|l|}{ Media } & 3,4 & 5,20 \\
\hline \multicolumn{2}{|c|}{ Desv. Estándar } & 0,97 & 1,26 & \multicolumn{2}{|c|}{ Desv. Estándar } & 0,99 & 1,16 & \multicolumn{2}{|c|}{ Desv. Estándar } & 1,26 & 1,31 \\
\hline \multicolumn{2}{|l|}{ Máximo } & 6,6 & 8,1 & \multicolumn{2}{|l|}{ Máximo } & 6,9 & 8,5 & \multicolumn{2}{|l|}{ Máximo } & 7,9 & 9,8 \\
\hline \multicolumn{2}{|l|}{ Mínimo } & 2,0 & 3,1 & \multicolumn{2}{|l|}{ Mínimo } & 2,3 & 3,6 & \multicolumn{2}{|l|}{ Mínimo } & 2,1 & 3,9 \\
\hline
\end{tabular}


Tabla 4.12: Rugosidad medida en tramo CR10 y CR11

\begin{tabular}{|c|c|c|c|c|c|}
\hline \multicolumn{3}{|c|}{ IRI (m/km) Tramo CR10 - Ruta B-169 } & \multicolumn{3}{|c|}{ IRI (m/km) Tramo CR11 - Ruta B-169 } \\
\hline \multicolumn{2}{|c|}{ Kilómetro } & \multirow{2}{*}{$\begin{array}{l}\text { Ene-06 } \\
(\mathrm{m} / \mathrm{km})\end{array}$} & \multicolumn{2}{|c|}{ Kilómetro } & \multirow{2}{*}{$\begin{array}{l}\text { Ene-06 } \\
(\mathrm{m} / \mathrm{km})\end{array}$} \\
\hline Inicial & Final & & Inicial & Final & \\
\hline 3,00 & 3,10 & 3,4 & 7,00 & 7,10 & 4,2 \\
\hline 3,10 & 3,20 & 4,1 & 7,10 & 7,20 & 4,1 \\
\hline 3,20 & 3,30 & 6,0 & 7,20 & 7,30 & 4,4 \\
\hline 3,30 & 3,40 & 4,2 & 7,30 & 7,40 & 4,4 \\
\hline 3,40 & 3,50 & 3,3 & 7,40 & 7,50 & 4,1 \\
\hline 3,50 & 3,60 & 3,2 & 7,50 & 7,60 & 4,5 \\
\hline 3,60 & 3,70 & 4,6 & 7,60 & 7,70 & 3,2 \\
\hline 3,70 & 3,80 & 2,9 & 7,70 & 7,80 & 5,6 \\
\hline 3,80 & 3,90 & 5,2 & 7,80 & 7,90 & 4,6 \\
\hline 3,90 & 4,00 & 3,9 & 7,90 & 8,00 & 5,4 \\
\hline 4,00 & 4,10 & 2,9 & 8,00 & 8,10 & 7,4 \\
\hline 4,10 & 4,20 & 2,6 & 8,10 & 8,20 & 7,0 \\
\hline 4,20 & 4,30 & 3,2 & 8,20 & 8,30 & 4,3 \\
\hline 4,30 & 4,40 & 2,0 & 8,30 & 8,40 & 5,6 \\
\hline 4,40 & 4,50 & 2,0 & 8,40 & 8,50 & 6,3 \\
\hline 4,50 & 4,60 & 2,0 & 8,50 & 8,60 & 3,9 \\
\hline 4,60 & 4,70 & 2,7 & 8,60 & 8,70 & 7,1 \\
\hline 4,70 & 4,80 & 2,0 & 8,70 & 8,80 & 6,0 \\
\hline 4,80 & 4,90 & 2,8 & 8,80 & 8,90 & 8,6 \\
\hline 4,90 & 5,00 & 4,3 & 8,90 & 9,00 & 5,9 \\
\hline 5,00 & 5,10 & 5,7 & 9,00 & 9,10 & 4,8 \\
\hline 5,10 & 5,20 & 4,8 & 9,10 & 9,20 & 5,5 \\
\hline 5,20 & 5,30 & 9,0 & 9,20 & 9,30 & 4,2 \\
\hline 5,30 & 5,40 & 4,7 & 9,30 & 9,40 & 3,7 \\
\hline 5,40 & 5,50 & 4,4 & 9,40 & 9,50 & 2,5 \\
\hline 5,50 & 5,60 & 3,6 & 9,50 & 9,60 & 3,8 \\
\hline 5,60 & 5,70 & 3,2 & 9,60 & 9,70 & 4,5 \\
\hline 5,70 & 5,80 & 4,1 & 9,70 & 9,80 & 4,4 \\
\hline 5,80 & 5,90 & 6,3 & 9,80 & 9,90 & 3,8 \\
\hline 5,90 & 6,00 & 7,0 & 9,90 & 10,00 & 3,9 \\
\hline \multicolumn{2}{|l|}{ Media } & 4,0 & \multicolumn{2}{|l|}{ Media } & 4,9 \\
\hline \multicolumn{2}{|c|}{ Desviación Estándar } & 1,61 & \multicolumn{2}{|c|}{ Desviación Estándar } & 1,36 \\
\hline \multicolumn{2}{|l|}{ Máximo } & 9,0 & \multicolumn{2}{|l|}{ Máximo } & 8,6 \\
\hline \multicolumn{2}{|l|}{ Mínimo } & 2,0 & \multicolumn{2}{|l|}{ Mínimo } & 2,5 \\
\hline
\end{tabular}


Tabla 4.13: Rugosidad medida en tramo CR12 y CR13

\begin{tabular}{|c|c|c|c|c|c|}
\hline \multicolumn{3}{|c|}{ IRI (m/km) Tramo CR12 - Ruta B-180 } & \multicolumn{3}{|c|}{ IRI (m/km) Tramo CR13 - Ruta B-180 } \\
\hline \multicolumn{2}{|c|}{ Kilómetro } & \multirow{2}{*}{$\begin{array}{l}\text { Ene-06 } \\
(\mathrm{m} / \mathrm{km})\end{array}$} & \multicolumn{2}{|c|}{ Kilómetro } & \multirow{2}{*}{$\begin{array}{l}\text { Ene-06 } \\
(\mathrm{m} / \mathrm{km}) \\
\end{array}$} \\
\hline Inicial & Final & & Inicial & Final & \\
\hline 13,00 & 13,10 & 7,8 & 25,00 & 25,10 & 5,3 \\
\hline 13,10 & 13,20 & 3,3 & 25,10 & 25,20 & 5,6 \\
\hline 13,20 & 13,30 & 3,5 & 25,20 & 25,30 & 7,0 \\
\hline 13,30 & 13,40 & 3,9 & 25,30 & 25,40 & 4,1 \\
\hline 13,40 & 13,50 & 3,6 & 25,40 & 25,50 & 5,2 \\
\hline 13,50 & 13,60 & 3,5 & 25,50 & 25,60 & 5,1 \\
\hline 13,60 & 13,70 & 2,7 & 25,60 & 25,70 & 3,7 \\
\hline 13,70 & 13,80 & 3,7 & 25,70 & 25,80 & 3,1 \\
\hline 13,80 & 13,90 & 3,9 & 25,80 & 25,90 & 3,3 \\
\hline 13,90 & 14,00 & 3,9 & 25,90 & 26,00 & 2,8 \\
\hline 14,00 & 14,10 & 4,1 & 26,00 & 26,10 & 2,5 \\
\hline 14,10 & 14,20 & 3,8 & 26,10 & 26,20 & 2,5 \\
\hline 14,20 & 14,30 & 3,6 & 26,20 & 26,30 & 2,4 \\
\hline 14,30 & 14,40 & 3,5 & 26,30 & 26,40 & 2,5 \\
\hline 14,40 & 14,50 & 3,2 & 26,40 & 26,50 & 2,4 \\
\hline 14,50 & 14,60 & 4,5 & 26,50 & 26,60 & 2,2 \\
\hline 14,60 & 14,70 & 3,6 & 26,60 & 26,70 & 3,4 \\
\hline 14,70 & 14,80 & 3,4 & 26,70 & 26,80 & 2,9 \\
\hline 14,80 & 14,90 & 3,9 & 26,80 & 26,90 & 3,0 \\
\hline 14,90 & 15,00 & 3,4 & 26,90 & 27,00 & 3,3 \\
\hline 15,00 & 15,10 & 3,0 & 27,00 & 27,10 & 3,1 \\
\hline 15,10 & 15,20 & 3,0 & 27,10 & 27,20 & 3,0 \\
\hline 15,20 & 15,30 & 3,0 & 27,20 & 27,30 & 2,8 \\
\hline 15,30 & 15,40 & 2,9 & 27,30 & 27,40 & 3,0 \\
\hline 15,40 & 15,50 & 4,8 & 27,40 & 27,50 & 3,0 \\
\hline 15,50 & 15,60 & 3,8 & 27,50 & 27,60 & 2,6 \\
\hline 15,60 & 15,70 & 2,7 & 27,60 & 27,70 & 2,8 \\
\hline 15,70 & 15,80 & 2,5 & 27,70 & 27,80 & 3,2 \\
\hline 15,80 & 15,90 & 2,8 & 27,80 & 27,90 & 3,0 \\
\hline 15,90 & 16,00 & 2,3 & 27,90 & 28,00 & 2,5 \\
\hline \multicolumn{2}{|l|}{ Media } & 3,6 & \multicolumn{2}{|l|}{ Media } & 3,4 \\
\hline \multicolumn{2}{|c|}{ Desviación Estándar } & 0,98 & \multicolumn{2}{|c|}{ Desviación Estándar } & 1,14 \\
\hline \multicolumn{2}{|l|}{ Máximo } & 7,8 & \multicolumn{2}{|l|}{ Máximo } & 7,0 \\
\hline \multicolumn{2}{|l|}{ Mínimo } & 2,3 & \multicolumn{2}{|l|}{ Mínimo } & 2,2 \\
\hline
\end{tabular}


Tabla 4.14: Rugosidad medida en tramo CR14 y CR15

\begin{tabular}{|c|c|c|c|c|c|}
\hline \multicolumn{3}{|c|}{ IRI (m/km) Tramo CR14 - Ruta B-207 } & \multicolumn{3}{|c|}{ IRI (m/km) Tramo CR15 - Ruta B-207 } \\
\hline \multicolumn{2}{|c|}{ Kilómetro } & \multirow{2}{*}{$\begin{array}{l}\text { Ene-06 } \\
(\mathrm{m} / \mathrm{km})\end{array}$} & \multicolumn{2}{|c|}{ Kilómetro } & \multirow{2}{*}{$\begin{array}{l}\text { Ene-06 } \\
(\mathrm{m} / \mathrm{km})\end{array}$} \\
\hline Inicial & Final & & Inicial & Final & \\
\hline 6,30 & 6,40 & 4,6 & 9,00 & 9,10 & 3,7 \\
\hline 6,40 & 6,50 & 4,6 & 9,10 & 9,20 & 3,3 \\
\hline 6,50 & 6,60 & 4,0 & 9,20 & 9,30 & 9,2 \\
\hline 6,60 & 6,70 & 2,8 & 9,30 & 9,40 & 5,5 \\
\hline 6,70 & 6,80 & 2,8 & 9,40 & 9,50 & 5,4 \\
\hline 6,80 & 6,90 & 3,6 & 9,50 & 9,60 & 3,6 \\
\hline 6,90 & 7,00 & 4,1 & 9,60 & 9,70 & 2,5 \\
\hline 7,00 & 7,10 & 4,0 & 9,70 & 9,80 & 3,0 \\
\hline 7,10 & 7,20 & 6,4 & 9,80 & 9,90 & 3,5 \\
\hline 7,20 & 7,30 & 11,2 & 9,90 & 10,00 & 3,3 \\
\hline 7,30 & 7,40 & 6,2 & 10,00 & 10,10 & 5,5 \\
\hline 7,40 & 7,50 & 6,0 & 10,10 & 10,20 & 4,3 \\
\hline 7,50 & 7,60 & 5,3 & 10,20 & 10,30 & 4,9 \\
\hline 7,60 & 7,70 & 4,0 & 10,30 & 10,40 & 5,6 \\
\hline 7,70 & 7,80 & 4,4 & 10,40 & 10,50 & 5,7 \\
\hline 7,80 & 7,90 & 3,5 & 10,50 & 10,60 & 5,2 \\
\hline 7,90 & 8,00 & 5,4 & 10,60 & 10,70 & 5,5 \\
\hline 8,00 & 8,10 & 4,7 & 10,70 & 10,80 & 5,4 \\
\hline 8,10 & 8,20 & 3,2 & 10,80 & 10,90 & 4,4 \\
\hline 8,20 & 8,30 & 3,8 & 10,90 & 11,00 & 4,4 \\
\hline 8,30 & 8,40 & 3,1 & 11,00 & 11,10 & 4,2 \\
\hline 8,40 & 8,50 & 3,2 & 11,10 & 11,20 & 4,6 \\
\hline 8,50 & 8,60 & 3,4 & 11,20 & 11,30 & 7,0 \\
\hline 8,60 & 8,70 & 4,0 & 11,30 & 11,40 & 4,8 \\
\hline 8,70 & 8,80 & 4,8 & 11,40 & 11,50 & 4,0 \\
\hline \multicolumn{2}{|l|}{ Media } & 4,5 & \multicolumn{2}{|l|}{ Media } & 4,7 \\
\hline \multicolumn{2}{|c|}{ Desviación Estándar } & 1,72 & \multicolumn{2}{|c|}{ Desviación Estándar } & 1,40 \\
\hline \multicolumn{2}{|l|}{ Máximo } & 11,2 & \multicolumn{2}{|l|}{ Máximo } & 9,2 \\
\hline \multicolumn{2}{|l|}{ Mínimo } & 2,8 & \multicolumn{2}{|l|}{ Mínimo } & 2,5 \\
\hline
\end{tabular}


Tabla 4.15: Rugosidad medida en tramo CR16 y CR17

\begin{tabular}{|c|c|c|c|c|c|c|c|}
\hline \multicolumn{4}{|c|}{ IRI (m/km) Tramo CR16 - Ruta B-400 } & \multicolumn{4}{|c|}{ IRI (m/km) Tramo CR17 - Ruta B-400 } \\
\hline \multicolumn{2}{|c|}{ Kilómetro } & \multirow{2}{*}{$\begin{array}{l}\text { Nov-05 } \\
\text { (m/km) }\end{array}$} & \multirow{2}{*}{$\begin{array}{l}\text { Ene-06 } \\
\text { (m/km) }\end{array}$} & \multicolumn{2}{|c|}{ Kilómetro } & \multirow{2}{*}{$\begin{array}{l}\text { Nov-05 } \\
\text { (m/km) }\end{array}$} & \multirow{2}{*}{$\begin{array}{l}\text { Ene-06 } \\
(\mathrm{m} / \mathrm{km})\end{array}$} \\
\hline Inicial & Final & & & Inicial & Final & & \\
\hline 1,00 & 1,10 & 3,4 & 3,6 & 6,00 & 6,10 & 3,7 & 4,2 \\
\hline 1,10 & 1,20 & 2,6 & 3,0 & 6,10 & 6,20 & 3,0 & 3,3 \\
\hline 1,20 & 1,30 & 3,0 & 3,2 & 6,20 & 6,30 & 3,6 & 4,1 \\
\hline 1,30 & 1,40 & 2,5 & 2,8 & 6,30 & 6,40 & 3,0 & 3,2 \\
\hline 1,40 & 1,50 & 2,9 & 3,2 & 6,40 & 6,50 & 3,3 & 3,5 \\
\hline 1,50 & 1,60 & 3,1 & 3,2 & 6,50 & 6,60 & 3,0 & 3,4 \\
\hline 1,60 & 1,70 & 2,5 & 3,1 & 6,60 & 6,70 & 3,7 & 3,8 \\
\hline 1,70 & 1,80 & 2,5 & 2,8 & 6,70 & 6,80 & 3,3 & 3,6 \\
\hline 1,80 & 1,90 & 2,6 & 2,8 & 6,80 & 6,90 & 3,5 & 3,7 \\
\hline 1,90 & 2,00 & 2,8 & 3,0 & 6,90 & 7,00 & 3,3 & 3,9 \\
\hline 2,00 & 2,10 & 3,0 & 3,1 & 7,00 & 7,10 & 4,0 & 4,7 \\
\hline 2,10 & 2,20 & 2,5 & 2,7 & 7,10 & 7,20 & 4,0 & 4,3 \\
\hline 2,20 & 2,30 & 2,9 & 3,2 & 7,20 & 7,30 & 3,9 & 4,0 \\
\hline 2,30 & 2,40 & 2,6 & 2,8 & 7,30 & 7,40 & 3,5 & 4,1 \\
\hline 2,40 & 2,50 & 2,4 & 3,2 & 7,40 & 7,50 & 3,7 & 4,0 \\
\hline 2,50 & 2,60 & 2,6 & 3,1 & 7,50 & 7,60 & 2,3 & 3,5 \\
\hline 2,60 & 2,70 & 3,5 & 3,7 & 7,60 & 7,70 & 3,0 & 3,3 \\
\hline 2,70 & 2,80 & 2,9 & 3,3 & 7,70 & 7,80 & 3,5 & 3,8 \\
\hline 2,80 & 2,90 & 3,3 & 3,5 & 7,80 & 7,90 & 3,3 & 3,7 \\
\hline 2,90 & 3,00 & 2,5 & 2,8 & 7,90 & 8,00 & 3,6 & 4,2 \\
\hline 3,00 & 3,10 & 2,9 & 3,6 & 8,00 & 8,10 & 3,3 & 4,5 \\
\hline 3,10 & 3,20 & 2,7 & 3,4 & 8,10 & 8,20 & 3,7 & 4,3 \\
\hline 3,20 & 3,30 & 3,7 & 3,9 & 8,20 & 8,30 & 4,2 & 4,4 \\
\hline 3,30 & 3,40 & 2,6 & 2,9 & 8,30 & 8,40 & 4,0 & 4,3 \\
\hline 3,40 & 3,50 & 3,4 & 6,7 & 8,40 & 8,50 & 3,9 & 4,3 \\
\hline 3,50 & 3,60 & 4,7 & 8,0 & 8,50 & 8,60 & 3,8 & 4,5 \\
\hline 3,60 & 3,70 & 3,3 & 6,5 & 8,60 & 8,70 & 4,5 & 5,5 \\
\hline 3,70 & 3,80 & 2,9 & 3,5 & 8,70 & 8,80 & 4,2 & 4,5 \\
\hline 3,80 & 3,90 & 3,1 & 3,2 & 8,80 & 8,90 & 3,4 & 3,9 \\
\hline 3,90 & 4,00 & 2,2 & 2,5 & 8,90 & 9,00 & 4,5 & 6,2 \\
\hline 4,00 & 4,10 & 2,2 & 3,6 & 9,00 & 9,10 & 5,6 & 8,3 \\
\hline 4,10 & 4,20 & 3 & 3,2 & 9,10 & 9,20 & 4,8 & 6,5 \\
\hline 4,20 & 4,30 & 3,6 & 3,8 & 9,20 & 9,30 & 3,6 & 5,3 \\
\hline 4,30 & 4,40 & 3 & 3,2 & 9,30 & 9,40 & 3,0 & 4,3 \\
\hline 4,40 & 4,50 & 2,8 & 3,1 & 9,40 & 9,50 & 4,2 & 4,5 \\
\hline 4,50 & 4,60 & 2,8 & 3,5 & 9,50 & 9,60 & 3,6 & 4,1 \\
\hline 4,60 & 4,70 & 2,9 & 3,5 & 9,60 & 9,70 & 3,3 & 3,4 \\
\hline 4,70 & 4,80 & 3 & 3,3 & 9,70 & 9,80 & 4,6 & 5,5 \\
\hline 4,80 & 4,90 & 3,5 & 3,5 & 9,80 & 9,90 & 4,0 & 4,2 \\
\hline 4,90 & 5,00 & 2,6 & 2,9 & 9,90 & 10,00 & 3,0 & 3,3 \\
\hline \multicolumn{2}{|l|}{ Media } & 2,9 & 3,50 & \multicolumn{2}{|l|}{ Media } & 3,7 & 4,30 \\
\hline \multicolumn{2}{|c|}{ Desviación Estándar } & 0,47 & 1,09 & \multicolumn{2}{|c|}{ Desviación Estándar } & 0,60 & 0,99 \\
\hline \multicolumn{2}{|l|}{ Máximo } & 4,7 & 8,0 & \multicolumn{2}{|l|}{ Máximo } & 5,6 & 8,3 \\
\hline \multicolumn{2}{|l|}{ Mínimo } & 2,2 & 2,5 & Mínimo & & 2,3 & 3,2 \\
\hline
\end{tabular}


Tabla 4.16: Rugosidad medida en tramo CR18 y CR19

\begin{tabular}{|c|c|c|c|c|c|c|c|}
\hline \multicolumn{4}{|c|}{ IRI (m/km) Tramo CR18 - Ruta B-400 } & \multicolumn{4}{|c|}{ IRI (m/km) Tramo CR19 - Ruta B-400 } \\
\hline \multicolumn{2}{|c|}{ Kilómetro } & \multirow{2}{*}{$\begin{array}{l}\text { Nov-05 } \\
\text { (m/km) }\end{array}$} & \multirow{2}{*}{$\begin{array}{l}\text { Ene-06 } \\
\text { (m/km) }\end{array}$} & \multicolumn{2}{|c|}{ Kilómetro } & \multirow[t]{2}{*}{ Nov-05 } & \multirow{2}{*}{$\begin{array}{l}\text { Ene-06 } \\
(\mathrm{m} / \mathrm{km})\end{array}$} \\
\hline Inicial & $(\mathrm{m} / \mathrm{km})$ & & & $(\mathrm{m} / \mathrm{km})$ & $(\mathrm{m} / \mathrm{km})$ & & \\
\hline 16,00 & 16,10 & 4,5 & 7,0 & 21,00 & 21,10 & 2,7 & 3,1 \\
\hline 16,10 & 16,20 & 4,0 & 5,1 & 21,10 & 21,20 & 2,6 & 2,8 \\
\hline 16,20 & 16,30 & 3,3 & 4,0 & 21,20 & 21,30 & 2,7 & 2,8 \\
\hline 16,30 & 16,40 & 2,9 & 3,4 & 21,30 & 21,40 & 2,6 & 2,7 \\
\hline 16,40 & 16,50 & 3,8 & 4,0 & 21,40 & 21,50 & 3,3 & 3,4 \\
\hline 16,50 & 16,60 & 3,3 & 3,2 & 21,50 & 21,60 & 2,8 & 2,9 \\
\hline 16,60 & 16,70 & 4,0 & 4,3 & 21,60 & 21,70 & 3,0 & 3,4 \\
\hline 16,70 & 16,80 & 2,2 & 2,6 & 21,70 & 21,80 & 3,8 & 4,5 \\
\hline 16,80 & 16,90 & 3,6 & 4,1 & 21,80 & 21,90 & 3,2 & 6,3 \\
\hline 16,90 & 17,00 & 2,6 & 3,2 & 21,90 & 22,00 & 3,5 & 5,1 \\
\hline 17,00 & 17,10 & 3,5 & 3,8 & 22,00 & 22,10 & 3,2 & 3,3 \\
\hline 17,10 & 17,20 & 3,1 & 3,5 & 22,10 & 22,20 & 3,0 & 3,3 \\
\hline 17,20 & 17,30 & 3,4 & 3,6 & 22,20 & 22,30 & 3,0 & 3,4 \\
\hline 17,30 & 17,40 & 3,0 & 3,3 & 22,30 & 22,40 & 2,8 & 2,8 \\
\hline 17,40 & 17,50 & 2,6 & 2,9 & 22,40 & 22,50 & 3,7 & 3,8 \\
\hline 17,50 & 17,60 & 2,4 & 2,6 & 22,50 & 22,60 & 3,7 & 3,7 \\
\hline 17,60 & 17,70 & 2,7 & 3,2 & 22,60 & 22,70 & 3,5 & 4,3 \\
\hline 17,70 & 17,80 & 2,7 & 2,9 & 22,70 & 22,80 & 3,0 & 3,2 \\
\hline 17,80 & 17,90 & 3,1 & 3,2 & 22,80 & 22,90 & 3,5 & 3,5 \\
\hline 17,90 & 18,00 & 2,7 & 2,9 & 22,90 & 23,00 & 3,1 & 3,2 \\
\hline 18,00 & 18,10 & 3,1 & 3,8 & 23,00 & 23,10 & 3,5 & 3,8 \\
\hline 18,10 & 18,20 & 3,1 & 3,6 & 23,10 & 23,20 & 3,2 & 3,2 \\
\hline 18,20 & 18,30 & 3,6 & 3,9 & 23,20 & 23,30 & 2,6 & 2,8 \\
\hline 18,30 & 18,40 & 3,3 & 3,8 & 23,30 & 23,40 & 2,7 & 2,8 \\
\hline 18,40 & 18,50 & 3,8 & 4,1 & 23,40 & 23,50 & 3,0 & 3,2 \\
\hline 18,50 & 18,60 & 2,6 & 2,9 & 23,50 & 23,60 & 3,4 & 3,8 \\
\hline 18,60 & 18,70 & 3,7 & 4,1 & 23,60 & 23,70 & 4,0 & 4,1 \\
\hline 18,70 & 18,80 & 3,0 & 3,4 & 23,70 & 23,80 & 3,8 & 3,9 \\
\hline 18,80 & 18,90 & 3,5 & 6,5 & 23,80 & 23,90 & 3,5 & 3,6 \\
\hline 18,90 & 19,00 & 5,3 & 8,0 & 23,90 & 24,00 & 3,4 & 3,4 \\
\hline 19,00 & 19,10 & 4,0 & 5,9 & 24,00 & 24,10 & 3,2 & 3,7 \\
\hline 19,10 & 19,20 & 2,9 & 4,9 & 24,10 & 24,20 & 3,3 & 3,6 \\
\hline 19,20 & 19,30 & 4,0 & 4,6 & 24,20 & 24,30 & 3,0 & 3,8 \\
\hline 19,30 & 19,40 & 3,4 & 3,7 & 24,30 & 24,40 & 3,5 & 4,7 \\
\hline 19,40 & 19,50 & 3,3 & 3,5 & 24,40 & 24,50 & 4,3 & 6,8 \\
\hline 19,50 & 19,60 & 3,1 & 3,3 & 24,50 & 24,60 & 3,0 & 4,7 \\
\hline 19,60 & 19,70 & 4,0 & 4,5 & 24,60 & 24,70 & 2,3 & 3,3 \\
\hline 19,70 & 19,80 & 2,4 & 2,5 & 24,70 & 24,80 & 2,9 & 2,8 \\
\hline 19,80 & 19,90 & 2,7 & 3,2 & 24,80 & 24,90 & 2,6 & 2,7 \\
\hline 19,90 & 20,00 & 2,6 & 3,1 & 24,90 & 25,00 & 2,4 & 2,5 \\
\hline \multicolumn{2}{|l|}{ Media } & 3,3 & 3,9 & \multicolumn{2}{|l|}{ Media } & 3,2 & 3,6 \\
\hline \multicolumn{2}{|c|}{ Desviación Estándar } & 0,64 & 1,19 & \multicolumn{2}{|c|}{ Desviación Estándar } & 0,46 & 0,91 \\
\hline \multicolumn{2}{|l|}{ Máximo } & 5,3 & 8,0 & \multicolumn{2}{|l|}{ Máximo } & 4,3 & 6,8 \\
\hline \multicolumn{2}{|l|}{ Mínimo } & 2,2 & 2,5 & Mínimo & & 2,3 & 2,5 \\
\hline
\end{tabular}


Tabla 4.17: Rugosidad medida en tramo CR20 y CR21

\begin{tabular}{|c|c|c|c|c|c|}
\hline \multicolumn{3}{|c|}{ IRI (m/km) Tramo CR20 - Ruta B-955 } & \multicolumn{3}{|c|}{ IRI (m/km) Tramo CR21 - Ruta B-955 } \\
\hline \multicolumn{2}{|c|}{ Kilómetro } & \multirow{2}{*}{$\begin{array}{l}\text { Ene-06 } \\
(\mathrm{m} / \mathrm{km})\end{array}$} & \multicolumn{2}{|c|}{ Kilómetro } & \multirow{2}{*}{$\begin{array}{l}\text { Ene-06 } \\
(\mathrm{m} / \mathrm{km})\end{array}$} \\
\hline Inicial & Final & & Inicial & Final & \\
\hline 43,00 & 43,10 & 9,4 & 46,00 & 46,10 & 7,0 \\
\hline 43,10 & 43,20 & 9,1 & 46,10 & 46,20 & 8,4 \\
\hline 43,20 & 43,30 & 10,5 & 46,20 & 46,30 & 7,7 \\
\hline 43,30 & 43,40 & 9,9 & 46,30 & 46,40 & 5,9 \\
\hline 43,40 & 43,50 & 10,1 & 46,40 & 46,50 & 8,6 \\
\hline 43,50 & 43,60 & 10,2 & 46,50 & 46,60 & 8,2 \\
\hline 43,60 & 43,70 & 9,8 & 46,60 & 46,70 & 6,1 \\
\hline 43,70 & 43,80 & 9,3 & 46,70 & 46,80 & 6,5 \\
\hline 43,80 & 43,90 & 9,1 & 46,80 & 46,90 & 6,9 \\
\hline 43,90 & 44,00 & 8,9 & 46,90 & 47,00 & 7,2 \\
\hline 44,00 & 44,10 & 8,3 & 47,00 & 47,10 & 6,2 \\
\hline 44,10 & 44,20 & 6,6 & 47,10 & 47,20 & 5,8 \\
\hline 44,20 & 44,30 & 7,3 & 47,20 & 47,30 & 6,0 \\
\hline 44,30 & 44,40 & 6,9 & 47,30 & 47,40 & 5,9 \\
\hline 44,40 & 44,50 & 4,0 & 47,40 & 47,50 & 5,7 \\
\hline 44,50 & 44,60 & 6,8 & 47,50 & 47,60 & 6,3 \\
\hline 44,60 & 44,70 & 7,3 & 47,60 & 47,70 & 4,1 \\
\hline 44,70 & 44,80 & 6,7 & 47,70 & 47,80 & 4,4 \\
\hline 44,80 & 44,90 & 8,6 & 47,80 & 47,90 & 4,0 \\
\hline 44,90 & 45,00 & 9,4 & 47,90 & 48,00 & 3,8 \\
\hline 45,00 & 45,10 & 10,5 & 48,00 & 48,10 & 3,9 \\
\hline 45,10 & 45,20 & 8,7 & 48,10 & 48,20 & 4,1 \\
\hline 45,20 & 45,30 & 8,7 & 48,20 & 48,30 & 3,7 \\
\hline 45,30 & 45,40 & 7,6 & 48,30 & 48,40 & 3,7 \\
\hline 45,40 & 45,50 & 6,4 & 48,40 & 48,50 & 3,7 \\
\hline 45,50 & 45,60 & 7,8 & 48,50 & 48,60 & 3,8 \\
\hline 45,60 & 45,70 & 6,1 & 48,60 & 48,70 & 7,7 \\
\hline 45,70 & 45,80 & 5,9 & 48,70 & 48,80 & 8,9 \\
\hline 45,80 & 45,90 & 9,0 & 48,80 & 48,90 & 9,8 \\
\hline 45,90 & 46,00 & 7,2 & 48,90 & 49,00 & 8,8 \\
\hline \multicolumn{2}{|l|}{ Media } & 8,2 & \multicolumn{2}{|l|}{ Media } & 6,1 \\
\hline \multicolumn{2}{|c|}{ Desviación Estándar } & 1,60 & \multicolumn{2}{|c|}{ Desviación Estándar } & 1,87 \\
\hline \multicolumn{2}{|l|}{ Máximo } & 10,5 & \multicolumn{2}{|l|}{ Máximo } & 9,80 \\
\hline \multicolumn{2}{|l|}{ Mínimo } & 4,0 & \multicolumn{2}{|l|}{ Mínimo } & 3,7 \\
\hline
\end{tabular}




\subsection{DATOS DE PÉRDIDA DE MATERIAL EN TRAMOS TESTIGOS}

\subsubsection{Valores medidos en tramos del estudio}

En el período de enero a julio del 2006 se procedió a realizar las mediciones según el plan predefinido, las que se desarrollaron sin contratiempos pero sí con una salvedad. La aprensión comentada en el capítulo tres, respecto al daño de la carpeta de rodado con la apertura de calicatas fue evidente, por tanto en los tramos de la ruta B-400 no se realizaron estas excavaciones, ya que ésta es una ruta que en el último tiempo se ha vuelto muy importante en el transporte de cobre de la Mina de Chuquicamata al Puerto de Mejillones.

Con el objeto de disminuir al mínimo el daño a la capa de rodado por la apertura de calicatas, el punto de medición cada vez fue el mismo, es decir, en cada oportunidad se abría la misma calicata para medir su espesor remanente, la que posteriormente se rellenaba con el mismo material y se compactaba, obviamente no pretendiendo reproducir las condiciones iniciales de consolidación de la capa de rodado.

No obstante, haber medido la pérdida de material por el método de calicatas, sólo en las rutas B-55 y B-207, estos no fueron considerados en el presente estudio por estimar que la metodología induce a un mayor error asociado a la operatoria. Los valores medidos son los indicados en la tabla 4.18.

Tabla 4.18: Pérdida de material - Método calicatas

\begin{tabular}{|l|c|c|c|c|c|c|c|c|}
\hline \multirow{2}{*}{ Fecha } & \multicolumn{5}{|c|}{ Espesor remanente de la carpeta (mm) } & \multirow{3}{*}{$\begin{array}{c}\text { Media } \\
\text { (mm) }\end{array}$} & $\begin{array}{c}\text { Desviación } \\
\text { Estándar }\end{array}$ \\
\cline { 2 - 9 } & \multicolumn{3}{|c|}{ B-55 } & \multicolumn{3}{c|}{ B-207 } & \\
\cline { 2 - 9 } & CPM4 & CPM5 & CPM6 & CPM7 & CPM8 & CPM9 & \\
\hline \hline Enero & 187 & 197 & 98 & 163 & 130 & 160 & 155.8 & 36.7 \\
\hline Marzo & 184 & 189 & 92 & 157 & 125 & 153 & 150.0 & 36.6 \\
\hline Mayo & 181 & 180 & 86 & 151 & 120 & 146 & 144.0 & 36.4 \\
\hline Julio & 178 & 171 & 78 & 144 & 115 & 139 & 137.5 & 37 \\
\hline Perdida Parcial & 8 & 27 & 20 & 19 & 15 & 21 & 18.3 & 6.3 \\
\hline Perdida Anual & 19 & 56 & 45 & 44 & 31 & 43 & 39.6 & 12.8 \\
\hline
\end{tabular}


En tanto que los valores medidos por el método topográfico, considerados en el presente estudio, para las rutas B-55, B-207 y B-400, son los indicados en la tabla 4.19. Los valores anuales de pérdida de material se obtuvieron a través de una función matemática.

Tabla 4.19: Pérdida de material $(\mathrm{mm})$ - Método topográfico $(\mathrm{mm})$

\begin{tabular}{|c|c|c|c|c|c|c|c|c|c|c|c|}
\hline \multirow{3}{*}{ FECHA } & \multicolumn{9}{|c|}{ Diferencia de cota (mm) } & \multirow{3}{*}{$\begin{array}{c}\text { Media } \\
(\mathrm{mm})\end{array}$} & \multirow{3}{*}{$\begin{array}{l}\text { Desv. } \\
\text { Estánda! }\end{array}$} \\
\hline & \multicolumn{3}{|c|}{ B-400 } & \multicolumn{3}{|c|}{ B-55 } & \multicolumn{3}{|c|}{ B-207 } & & \\
\hline & CPM1 & CPM2 & CPM3 & CPM4 & CPM5 & CPM6 & CPM7 & CPM8 & CPM9 & & \\
\hline Enero - Marzo & -6 & 20 & 5 & 9 & 4 & 8 & 10 & 1 & 2 & 5.9 & 7.2 \\
\hline Marzo - Mayo & 17 & -9 & 6 & 6 & 11 & 6 & -2 & 7 & 6 & 5.3 & 7.3 \\
\hline Mayo - Julio & 6 & 6 & 6 & 6 & 6 & 5 & 4 & 4 & 4 & 5.2 & 0.9 \\
\hline Perd. Parcial (mm) & 17 & 17 & 17 & 21 & 21 & 19 & 12 & 12 & 12 & 16.4 & 3.6 \\
\hline Perd. Anual (mm) & 23 & 12 & 45 & 42 & 35 & 34 & 16 & 21 & 20 & 27.5 & 11.7 \\
\hline
\end{tabular}

\subsubsection{Sumario del análisis de la metodología de medición}

Como conclusión a esta etapa de medición, se puede decir, que una vez analizados los resultados obtenidos y contrastadas las posibilidades que confieren ambos métodos en terreno, es mucho más recomendable el uso del taquímetro. Las principales razones para esta elección se sustentan en el hecho que la perforación de la carpeta genera una discontinuidad superficial en el camino, situación que obliga al conductor a esquivar esta singularidad y con ello el punto de medición, cambiando así las condiciones iniciales de tránsito y de medición del punto. Por otra parte, el hecho de hacer las calicatas conlleva a una dificulta operativa mayor, en términos de trabajo realizado, tiempo invertido y de la posibilidad de hacer un mayor número de observaciones. Además, cada pique es un bache en potencia que termina por dañar la carpeta más que ayudar a predecir su comportamiento.

A pesar de no contar con la certeza de medir en cada oportunidad en el punto exacto, el método topográfico entrega resultados con un mayor grado de precisión, una menor dispersión de los datos tomados, y por último, da la 
oportunidad de tomar una mayor cantidad de observaciones en un menor tiempo y sin producir alteraciones en la capa de rodado.

La tabla siguiente muestra los valores para el análisis de pérdida de material en caminos de la Región de Antofagasta.

Tabla 4.20: Valores para el análisis de pérdida de material

\begin{tabular}{|c|c|c|c|c|}
\hline \multicolumn{7}{|c|}{ PERDIDA DE MATERIAL (mm) } \\
\hline TRAM0 & $\begin{array}{c}\text { TMDA } \\
\text { veh/día }\end{array}$ & $\begin{array}{c}\text { MMP } \\
\text { mm/mes }\end{array}$ & $\begin{array}{c}\text { PERD. PARCIAL } \\
\text { mm }\end{array}$ & $\begin{array}{c}\text { PERD. ANUAL } \\
\text { mm/año }\end{array}$ \\
\hline CPM1 & 131 & 0,14 & 17 & 23 \\
\hline CPM2 & 132 & 0,14 & 17 & 13 \\
\hline CPM3 & 133 & 0,14 & 17 & 45 \\
\hline CPM4 & 20 & 0,48 & 21 & 42 \\
\hline CPM5 & 21 & 0,48 & 21 & 35 \\
\hline CPM6 & 22 & 0,48 & 19 & 34 \\
\hline CPM7 & 190 & 0,14 & 12 & 16 \\
\hline CPM8 & 190 & 0,14 & 12 & 21 \\
\hline CPM9 & 190 & 0,14 & 12 & 20 \\
\hline
\end{tabular}

\subsection{ANALISIS DE RUGOSIDAD EN LOS TRAMOS TESTIGOS}

\subsubsection{Rugosidad mínima medida en terreno}

Recordemos que la rugosidad mínima corresponde a la cota inferior de la rugosidad alcanzada por un camino dada ciertas condiciones granulométricas de la capa de rodado, el tránsito y las condiciones climáticas.

Del conjunto de datos expuestos en la tabla 4.21 se puede apreciar que la rugosidad mínima medida corresponde a:

\section{Rugosidad Mínima Medida = 2,0 m/km IRI}

Además no se aprecia una relación directa entre la rugosidad mínima y el tamaño máximo de partícula o el tránsito medio diario anual (TMDA). 
Tabla 4.21: Rugosidades mínimas

\begin{tabular}{|c|c|c|c|}
\hline Tramo & $\begin{array}{c}\text { Rugosidad - IRI } \\
\mathrm{m} / \mathrm{km}\end{array}$ & $\begin{array}{c}\text { Tamaño Máximo } \\
\text { mm }\end{array}$ & $\begin{array}{c}\text { TMDA } \\
\text { veh/día }\end{array}$ \\
\hline CR1 & 2,3 & 40 & 66 \\
\hline CR2 & 2,5 & 40 & 66 \\
\hline CR3 & 4,0 & 25 & 126 \\
\hline CR4 & 3,0 & 25 & 126 \\
\hline CR5 & 2,5 & 25 & 17 \\
\hline CR6 & 2,8 & 25 & 17 \\
\hline CR7 & 2,0 & 50 & 29 \\
\hline CR8 & 2,3 & 50 & 30 \\
\hline CR9 & 2,1 & 50 & 30 \\
\hline CR10 & 2,0 & 25 & 22 \\
\hline CR11 & 2,5 & 25 & 22 \\
\hline CR12 & 2,3 & 20 & 319 \\
\hline CR13 & 2,2 & 20 & 319 \\
\hline CR14 & 2,8 & 40 & 20 \\
\hline CR15 & 2,5 & 40 & 20 \\
\hline CR16 & 2,2 & 50 & 190 \\
\hline CR17 & 2,3 & 50 & 190 \\
\hline CR18 & 2,2 & 50 & 190 \\
\hline CR19 & 2,3 & 50 & 190 \\
\hline CR20 & 4,0 & 50 & 62 \\
\hline CR21 & 3,7 & 50 & 62 \\
\hline Media & 2,6 & 36,1 & 99,9 \\
\hline Desviación Estándar & 0,61 & 12,07 & 102,17 \\
\hline Máximo & 4,0 & 50,0 & 319,0 \\
\hline Mínimo & 2,0 & 20,0 & 17,0 \\
\hline
\end{tabular}

\subsubsection{Rugosidad máxima medida en terreno}

Las rugosidades máximas medidas en cada uno de los tramos del estudio se detallan en la tabla siguiente: 
Tabla 4.22: Rugosidad máxima

\begin{tabular}{|c|c|c|c|}
\hline Tramo & $\begin{array}{c}\text { Rugosidad - IRI } \\
\mathrm{m} / \mathrm{km}\end{array}$ & $\begin{array}{c}\text { Tamaño Máximo } \\
\text { mm }\end{array}$ & $\begin{array}{c}\text { TMDA } \\
\text { veh/día }\end{array}$ \\
\hline CR1 & 12,1 & 40 & 66 \\
\hline CR2 & 13,2 & 40 & 66 \\
\hline CR3 & 10,4 & 25 & 126 \\
\hline CR4 & 10,2 & 25 & 126 \\
\hline CR5 & 7,9 & 25 & 17 \\
\hline CR6 & 8,2 & 25 & 17 \\
\hline CR7 & 8,1 & 50 & 29 \\
\hline CR8 & 8,5 & 50 & 30 \\
\hline CR9 & 9,8 & 50 & 30 \\
\hline CR10 & 9,0 & 25 & 22 \\
\hline CR11 & 8,6 & 25 & 22 \\
\hline CR12 & 7,8 & 20 & 319 \\
\hline CR13 & 7,0 & 20 & 319 \\
\hline CR14 & 11,2 & 40 & 20 \\
\hline CR15 & 9,2 & 40 & 20 \\
\hline CR16 & 8,0 & 50 & 190 \\
\hline CR17 & 8,3 & 50 & 190 \\
\hline CR18 & 8,0 & 50 & 190 \\
\hline CR19 & 6,8 & 50 & 190 \\
\hline CR20 & 10,5 & 50 & 62 \\
\hline CR21 & 9,8 & 50 & 62 \\
\hline Media & 9,2 & 36,1 & 99,9 \\
\hline Desviación Estándar & 1,65 & 12,07 & 102,17 \\
\hline Máximo & 13,2 & 50,0 & 319,0 \\
\hline Mínimo & 6,8 & 20,0 & 17,0 \\
\hline
\end{tabular}

Se puede apreciar en la tabla que la máxima rugosidad alcanzada en las mediciones hechas en el seguimiento de los tramos en estudio corresponde a:

Rugosidad Máxima Medida = 13,2 IRI m/Km

\subsubsection{Rugosidad media de cada tramo}

Si bien es cierto, la rugosidad media en la realidad corresponde a un promedio 
de la rugosidad entre perfilados, en nuestro estudio al no existir estrategias de mantenimiento y conservación para estas rutas, la rugosidad media que se indica en la tabla siguiente, corresponde al promedio de la última medición de la rugosidad en cada tramo.

Tabla 4.23: Rugosidad media de cada tramo

\begin{tabular}{|c|c|c|c|}
\hline Tramo & $\begin{array}{c}\text { Rugosidad - IRI } \\
\mathrm{m} / \mathrm{km}\end{array}$ & $\begin{array}{c}\text { Tamaño Máximo } \\
\text { mm }\end{array}$ & $\begin{array}{c}\text { TMDA } \\
\text { veh/día }\end{array}$ \\
\hline CR1 & 6,7 & 40 & 66 \\
\hline CR2 & 7,7 & 40 & 66 \\
\hline CR3 & 7,8 & 25 & 126 \\
\hline CR4 & 5,5 & 25 & 126 \\
\hline CR5 & 3,9 & 25 & 17 \\
\hline CR6 & 4,1 & 25 & 17 \\
\hline CR7 & 4,4 & 50 & 29 \\
\hline CR8 & 5,1 & 50 & 30 \\
\hline CR9 & 5,2 & 50 & 30 \\
\hline CR10 & 4,0 & 25 & 22 \\
\hline CR11 & 4,9 & 25 & 22 \\
\hline CR12 & 3,6 & 20 & 319 \\
\hline CR13 & 3,4 & 20 & 319 \\
\hline CR14 & 4,5 & 40 & 20 \\
\hline CR15 & 4,7 & 40 & 20 \\
\hline CR16 & 3,5 & 50 & 190 \\
\hline CR17 & 4,3 & 50 & 190 \\
\hline CR18 & 3,9 & 50 & 190 \\
\hline CR19 & 3,6 & 50 & 190 \\
\hline CR20 & 8,2 & 50 & 62 \\
\hline CR21 & 6,1 & 50 & 62 \\
\hline Media & 5,0 & 36,1 & 99,9 \\
\hline Desviación Estándar & 1,48 & 12,07 & 102,17 \\
\hline Máximo & 8,2 & 50,0 & 319,0 \\
\hline Mínimo & 3,4 & 20,0 & 17,0 \\
\hline
\end{tabular}

Algunos resultados importantes de este análisis corresponden a los valores alcanzados para la media, desviación estándar, máxima y mínima rugosidad, los cuales, además se muestran en la gráfica siguiente: 


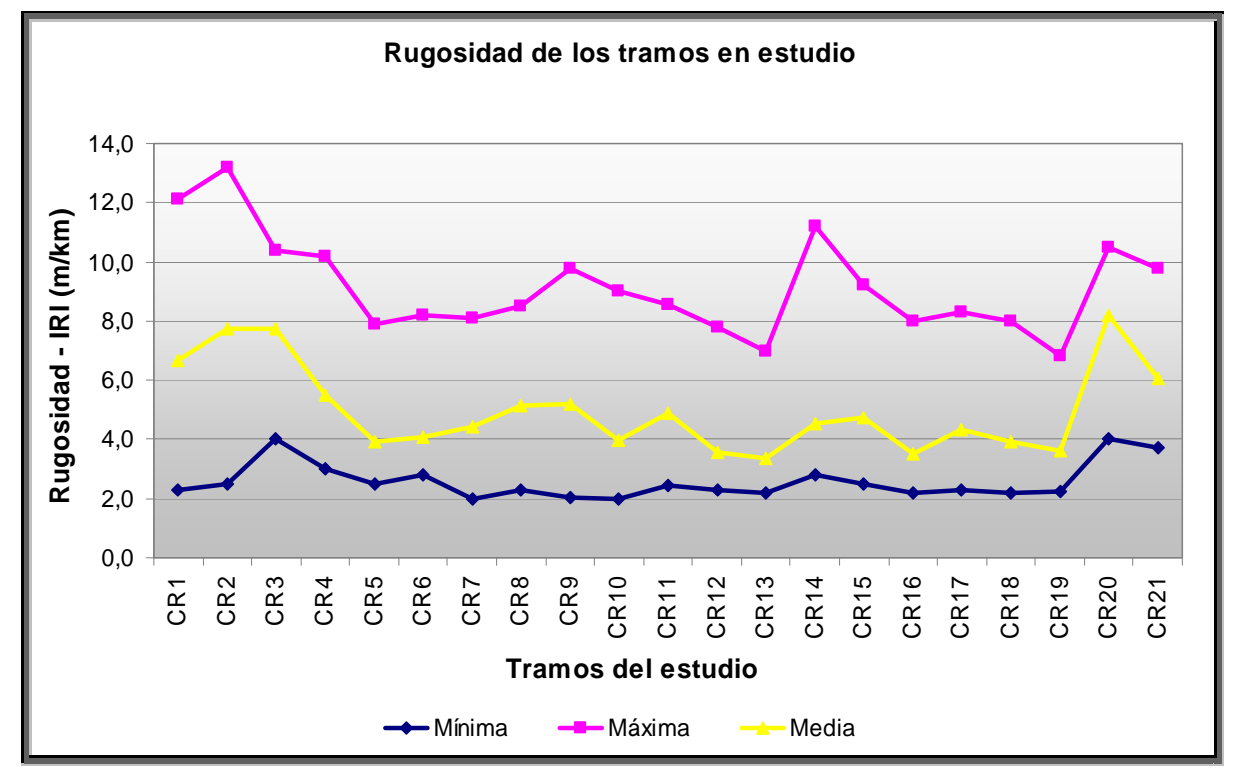

Figura 4.1: Grafica de rugosidad máx., mín. y media

\subsubsection{Análisis de rugosidad antes y después de un reperfilado}

Los caminos no pavimentados considerados en el presente estudios y que corresponden al programa de gobierno "Caminos Básicos 5000", son rutas que fueron diseñadas siguiendo la metodología AASHTO, con horizontes de vida sobre los tres años. La construcción de estas rutas, se iniciaron a mediados del año 2003, por tanto a la fecha de esta investigación ninguna de estas vías ha recibido trabajo alguno de conservación. En consecuencia, no fue posible medir la variable rugosidad antes y después del reperfilado.

\subsubsection{Rugosidad en las estaciones climáticas}

En el capítulo tres (3.7.3) se explicó detalladamente la particularidad pluviométrica de la Región de Antofagasta, la que geográficamente se ubica en el Desierto de Atacama, el más árido del mundo. Esta singularidad asociada a la errática recurrencia de las lluvias en la zona y la poca extensión en el tiempo de este estudio, confabularon para no hacer posible la obtención de mediciones de rugosidad en períodos de lluvia.

En definitiva, no fue posible obtener una apreciación comparativa del comportamiento de los caminos bajo régimen de lluvias versus régimen seco para la Región de Antofagasta. 


\subsection{AJUSTE DE LOS MODELOS DE DETERIORO}

En el presente acápite se persigue ajustar los modelos de deterioro del HDM-4 a las reales y particulares condiciones de la Región de Antofagasta de Chile, de acuerdo a lo observado en la zona propósito del estudio, siguiendo los procedimientos detallados en el capítulo tres.

Las relaciones del HDM-III que predicen el deterioro de los caminos no pavimentados y los efectos del mantenimiento, se han mantenido íntegramente en el HDM-4. Sin embargo, en atención a que en el HDM-III las unidades de rugosidad se especificaron en términos del "Índice Cuarto Carro", QI, y que la rugosidad en el HDM-4 se especifica como IRI (m/km), es que la nueva versión del HDM ha reformulado las relaciones de los modelos de deterioro declaradas por Paterson ${ }^{5}$ y Watanatada ${ }^{6}$ para reflejar la rugosidad en unidades de IRI.

\subsubsection{Ajuste del modelo de Rugosidad Máxima}

En el ajuste de los modelos de rugosidad se mantuvo la forma funcional del modelo entregados por Paterson ${ }^{7}$ y reformulado en el HDM-4, efectuando modificaciones en los parámetros que acompañan a las variables independientes comprendidas en el modelo.

Se explicita el modelo del HDM-4 para la variable dependiente Rugosidad máxima $\left(\mathrm{RI}_{\text {máx }}\right)$ como lineal en los parámetros y su expresión es la siguiente ${ }^{8}$ :

$$
\hat{R} I_{\max }=21,5-32,4 *(0.5-M G D)^{2}+0.017 *(H C)-0,764 *(R F) *(M M P / 1000)
$$

Donde:

$$
\mathrm{HC}=\text { Curvatura horizontal promedio del camino, en grados } / \mathrm{km}
$$

\footnotetext{
${ }^{5}$ William Paterson D.O. (1987). "Road Deterioration and Maintenance Effects: Models for Planning and Management. Highway Desing and Maintenance Standards Series", World Bank Transportation department, Washington D.C.

6 Watanatada et al. (1987), The Highway Design and Maintenance Standards Model, Volume 1 Description, The World Bank, John Hopkins University Press.

${ }^{7}$ William Paterson D.O. (1987). "Road Deterioration and Maintenance Effects: Models for Planning and Management. Highway Desing and Maintenance Standards Series", World Bank Transportation department, Washington D.C.

${ }^{8}$ Se explicita que las ecuaciones con gorro (hat) son las estimadas, en tanto que aquellas sin él, son las calculadas directamente de las variables recolectadas.
} 


$$
\begin{aligned}
& \mathrm{RF}=\text { Promedio de subidas más bajadas del camino, en } \mathrm{m} / \mathrm{km} \\
& M G D=\text { Relación del material en gradación polvo } \\
& M G D=1 \quad \text { si: } \mathrm{P} 425=0 \\
& M G D=P 075 / P 425 \quad \text { si: } \mathrm{P} 425>0 \\
& \text { P425 }=\text { Cantidad de material que pasa el tamiz } 0,425, \% \text { de la masa } \\
& \text { P075 }=\text { Cantidad de material que pasa el tamiz } 0,075, \% \text { de la masa } \\
& M M P=\text { Precipitación media mensual, en } \mathrm{mm} / \mathrm{mes}
\end{aligned}
$$

De la linealidad del algoritmo podemos decir que si las variables independientes fuesen cero, el menor valor que alcanzaría la variable dependiente sería considerablemente mayor que cero. Lo que permite proyectar linealmente la rugosidad máxima sin riego significativo de subestimación.

Las variables independientes no presentan correlaciones estadísticamente significativas entre ellas, lo que permite la aplicación del análisis de regresión y avala la estabilidad de los coeficientes estimados del modelo. La matriz de correlación siguiente muestra que ninguna resulta estadísticamente distinta de cero.

Tabla 4.24: Correlaciones entre cada variable para 21 muestras

\begin{tabular}{|c|c|c|c|c|}
\hline \multicolumn{2}{|c|}{ Variables } & HC & $(\mathbf{0 . 5}-\mathbf{M G D})^{\mathbf{2}}$ & RF * MMP/1000 \\
\hline \multirow{2}{*}{ HC } & Correlación & & $-0,2652$ & -0.0788 \\
\cline { 2 - 5 } & p-valor & & 0,245 & 0,7342 \\
\hline \multirow{2}{*}{$(\mathbf{0 . 5}-\mathbf{M G D )})^{2}$} & Correlación & $-0,2652$ & & 0,1622 \\
\cline { 2 - 5 } & p-valor & 0,245 & & 0,4825 \\
\hline $\begin{array}{c}\text { RF } \\
\text { MMP/1000 }\end{array}$ & Correlación & $-0,0788$ & 0,1622 & \\
\cline { 2 - 5 } & p-valor & 0,7342 & 0,4825 & \\
\hline
\end{tabular}

Esto refuerza la idea, por ejemplo, que la variable grado de curvatura (curvas del camino - HC) sería independiente de la variable subidas y bajadas (pendientes del camino - RF), de igual forma, la gradación de polvo (relación 
entre el material retenido en la malla 200 y la malla 40 - MGD), sería independiente de los valores de pendiente y de curvatura del camino.

En consecuencia, la ecuación de la variable dependiente "Rugosidad Máxima -

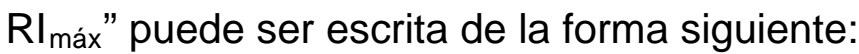

$$
R I_{\max }=K_{1}+K_{2} *(0,5-M G D)^{2}+K_{3}{ }^{*} H C+K_{4}{ }^{*} R F^{*} M M P+e
$$

Donde: "Ki" son los parámetros por determinar y "e" es el error aleatorio que representa todas aquellas otras condiciones que afectan las condiciones del camino y que no son capturadas por el modelo. Los parámetros estimados del Modelo HDM-4 y sus respectivos estadísticos se presentan en la tabla 4.25.

Tabla 4.25: Variable Dependiente: "RI $\mathrm{max}_{\text {" }}$

\begin{tabular}{|l|c|c|c|c|}
\hline \multicolumn{1}{|c|}{ Parámetro } & Estimación & Error estándar & Estadístico T & P-valor \\
\hline Constante & 9,41466 & 0,56808 & 16,5728 & 0,000 \\
\hline$(0,5-M G D)^{2}$ & $-65,0699$ & 19,8819 & $-3,27283$ & 0,0045 \\
\hline HC & 0,0163323 & 0,00753278 & 2,16817 & 0,0446 \\
\hline RF`MMP/1000 $^{*}$ & 59,9149 & 24,8411 & 2,41192 & 0,0275 \\
\hline
\end{tabular}

Adicionalmente, en la tabla 4.26 se muestra el análisis de varianza que permite rechazar la hipótesis nula de que todos los parámetros son iguales a cero, y por lo tanto aseverar que al menos uno de los parámetros del modelo sería significativamente distinto de cero. Esto sería congruente con los resultados de la tabla 4.25, que muestra que las tres variables del modelo son estadísticamente significativa en la explicación de las variaciones de la rugosidad máxima de los caminos no pavimentados de la región de Antofagasta ( $p$-valor $<0.05)$.

Tabla 4.26: Análisis de la Varianza

\begin{tabular}{|l|c|c|c|c|c|}
\hline \multicolumn{1}{|c|}{ Fuente } & $\begin{array}{c}\text { Suma } \\
\text { cuadrados }\end{array}$ & G.L. & $\begin{array}{c}\text { Cuadrado } \\
\text { Medio }\end{array}$ & Cociente F & P-valor \\
\hline Modelo & 31,4012 & 3 & 10,4671 & 7,78 & 0,0018 \\
\hline Residuo & 22,8816 & 17 & 1,34598 & & \\
\hline Total (Correl.) & $\mathbf{5 4 , 2 8 2 9}$ & $\mathbf{2 0}$ & & & \\
\hline
\end{tabular}


La estimación del modelo para describir la relación entre la variable dependiente $\mathrm{RI}_{\max }$ y las tres variables independientes, queda de la forma siguiente:

$$
\hat{\mathbf{R}} \mathbf{I}_{\max }=9,4146-65,069 *(0,5-M G D)^{2}+0,016332^{*} H C+59,914 * R F * M M P / 1000
$$

El modelo estimado tiene una capacidad de explicar el $51 \%$ de las variaciones de la variable dependiente $\left(R^{2}\right.$ ajustado $\left.=0,51\right)$, lo que sería significativamente mayor al ajuste alcanzado por Paterson en su modelo para Brasil.

Adicionalmente los signos encontrados para las variables gradación de polvo $\left((0,5-M G D)^{2}\right)$ y grado de curvatura $(H C)$, son similares a los esperados y a los obtenidos por Parterson y Watanatada para Brasil. Sin embargo, el signo para la variable pendiente del camino $\left(\mathrm{RF}^{\star} \mathrm{MMP} / 1000\right)$ es contrario y significativo al esperado. Aunque esto pudiese parecer una debilidad del modelo estimado respecto al modelo con el cual se está comparando, para la desértica región de Antofagasta tiene una explicación plausible, debido a que en el estudio para Brasil se podría esperar que las pendientes de los caminos no pavimentados, redujeran la rugosidad causadas por las precipitaciones. Esto implica que para Brasil el efecto capturado por esta variable esté asociado principalmente a la cantidad de agua caída.

En contraste, la pluviosidad en la región de Antofagasta es muy baja, lo que hace que el efecto del agua caída sobre el camino sea marginal y que el efecto capturado por está variable esté dominado por la pendiente de los caminos. Mostrando que la rugosidad será mayor, mientras mayor sean las pendientes. Lo que contrasta con las zonas lluviosas donde los caminos no pavimentados tienen mayor deterioro en los sectores planos producto de las precipitaciones, y donde las pendientes juegan un rol de protección debido a que evitan la acumulación de agua sobre la superficie del camino.

La Figura 4.2 denota una varianza homoscedástica, al mostrar valores positivos y negativos de los errores estimados, encontrándose la mayoría relativamente 
cercanos a cero. No evidenciando un comportamiento sistemático de los errores alrededor de su valor esperado o cero, con una distribución uniforme y similar dispersión. Por tanto, la figura evidencia que no existen indicios de heterocedasticidad en los errores de estimación del modelo, lo que es una condición para la aplicación de los modelos de regresión lineal.

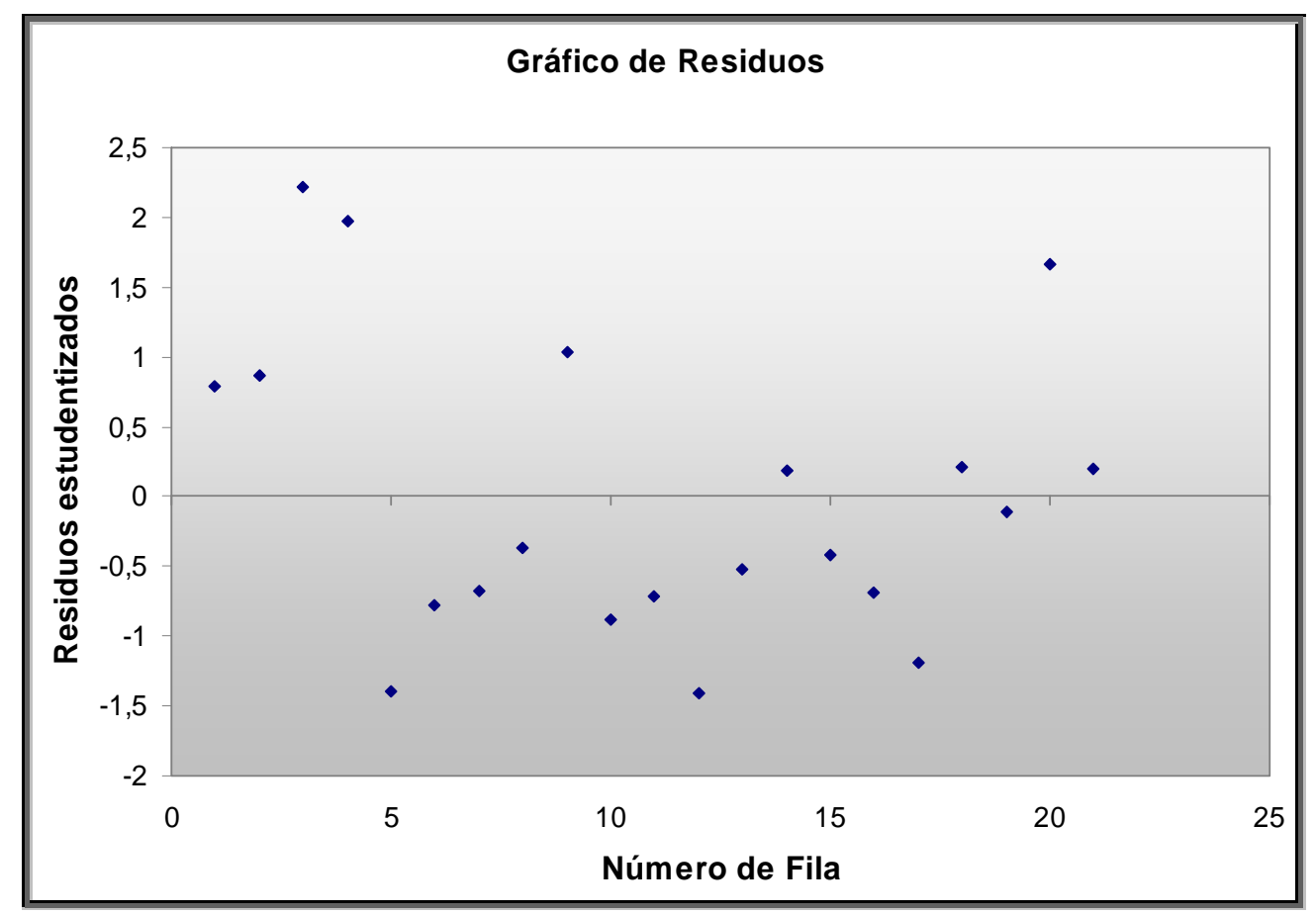

Figura 4.2: Gráfica de residuos - $\mathbf{R} \mathbf{I}_{\max }$

En análisis de regresión de datos de corte transversal en general, la autocorrelación o correlación serial no sería relevante, ya que no existe una dependencia temporal. De todas maneras, se estimó el índice de Durbin y Watson para medir autocorrelación y este resultó ser mayor a dos $(2,40848)$, indicando la no presencia de autocorrelación serial.

En la tabla 4.27 se resumen los valores medidos de las variables independientes, utilizadas en la estimación del modelo, curvatura horizontal, pendientes, pluviometría, gradación de polvo y de las rugosidades medidas en terreno, además de las estimadas por el modelo ajustado.

En la siguiente tabla podemos observar que la variable dependiente $\left(R I_{\max }\right.$ Ajustada), se encuentra lejos de valores negativos, es más, los valores que se 
obtienen están dentro del rango esperado para la rugosidad máxima (en caminos no pavimentados de la región) y de aquellos valores medidos en terreno.

Tabla 4.27: Determinación Rugosidad Máxima

\begin{tabular}{|l|c|c|c|c|c|c|c|c|c|}
\hline \multirow{2}{*}{ Tramo } & \multirow{2}{*}{$\begin{array}{c}\text { HC } \\
(\mathbf{\%} / \mathrm{km})\end{array}$} & $\begin{array}{c}\text { RF } \\
(\mathrm{m} / \mathrm{km})\end{array}$ & $\begin{array}{c}\text { MMP } \\
\mathrm{mm} / \mathrm{mes}\end{array}$ & $\begin{array}{c}\text { MGD } \\
\text { P075/P425 }\end{array}$ & $\begin{array}{l}\text { P075 } \\
\# \mathbf{2 0 0}\end{array}$ & $\begin{array}{c}\text { P425 } \\
\text { \# 40 }\end{array}$ & \multicolumn{3}{|c|}{ RImax m/km } \\
\hline CR1 & 28.0 & 17 & 1.43 & 0.5152 & 17 & 33 & 21.9 & 12.1 & 11.3 \\
\hline CR2 & 92.7 & 17 & 1.43 & 0.4722 & 17 & 36 & 23.0 & 13.2 & 12.3 \\
\hline CR3 & 8.0 & 5 & 0.14 & 0.6471 & 22 & 34 & 20.9 & 10.4 & 8.2 \\
\hline CR4 & 10.7 & 5 & 0.14 & 0.6471 & 22 & 34 & 21.0 & 10.2 & 8.2 \\
\hline CR5 & 0.0 & 8 & 1.43 & 0.3889 & 14 & 36 & 21.1 & 7.9 & 9.3 \\
\hline CR6 & 0.0 & 8 & 1.43 & 0.3684 & 14 & 38 & 20.9 & 8.2 & 9.0 \\
\hline CR7 & 3.0 & 20 & 1.43 & 0.3077 & 8 & 26 & 20.3 & 8.1 & 8.8 \\
\hline CR8 & 9.0 & 20 & 1.43 & 0.3077 & 8 & 26 & 20.4 & 8.5 & 8.9 \\
\hline CR9 & 3.0 & 20 & 1.43 & 0.3077 & 8 & 26 & 20.3 & 9.8 & 8.8 \\
\hline CR10 & 47.7 & 5 & 1.43 & 0.6071 & 17 & 28 & 21.9 & 9.0 & 9.9 \\
\hline CR11 & 7.7 & 5 & 1.43 & 0.6000 & 18 & 30 & 21.3 & 8.6 & 9.3 \\
\hline CR12 & 5.0 & 5 & 0.14 & 0.5714 & 20 & 35 & 21.4 & 7.8 & 9.2 \\
\hline CR13 & 13.0 & 5 & 0.14 & 0.6818 & 30 & 44 & 20.6 & 7.0 & 7.5 \\
\hline CR14 & 128.8 & 5 & 0.48 & 0.6000 & 21 & 35 & 23.4 & 11.2 & 11.0 \\
\hline CR15 & 69.2 & 5 & 0.48 & 0.6286 & 22 & 35 & 22.1 & 9.2 & 9.6 \\
\hline CR16 & 13.0 & 33 & 0.14 & 0.3636 & 8 & 22 & 21.1 & 8.0 & 8.7 \\
\hline CR17 & 65.8 & 25 & 0.14 & 0.3636 & 8 & 22 & 22.0 & 8.3 & 9.5 \\
\hline CR18 & 54.5 & 22 & 0.14 & 0.2963 & 8 & 27 & 21.1 & 8.0 & 7.8 \\
\hline CR19 & 2.0 & 20 & 0.14 & 0.2963 & 8 & 27 & 20.2 & 6.8 & 6.9 \\
\hline CR20 & 0.0 & 9 & 0.14 & 0.4000 & 12 & 30 & 21.2 & 10.5 & 8.8 \\
\hline CR21 & 11.3 & 9 & 0.14 & 0.5333 & 16 & 30 & 21.7 & 9.8 & 9.6 \\
\hline
\end{tabular}

En la figura 4.3 se pueden notar las diferencias que presentan las predicciones de los modelos de Paterson, para Brasil con datos para la región de Antofagasta, y el modelo ajustado para Antofagasta, versus los datos medidos en terreno. En la figura se puede apreciar que el modelo de Paterson se mantiene por sobre el valor real, distando bastante de lo medido en terreno. En tanto, el modelo estimado se ajusta considerablemente mejor a los datos medidos en terreno. 


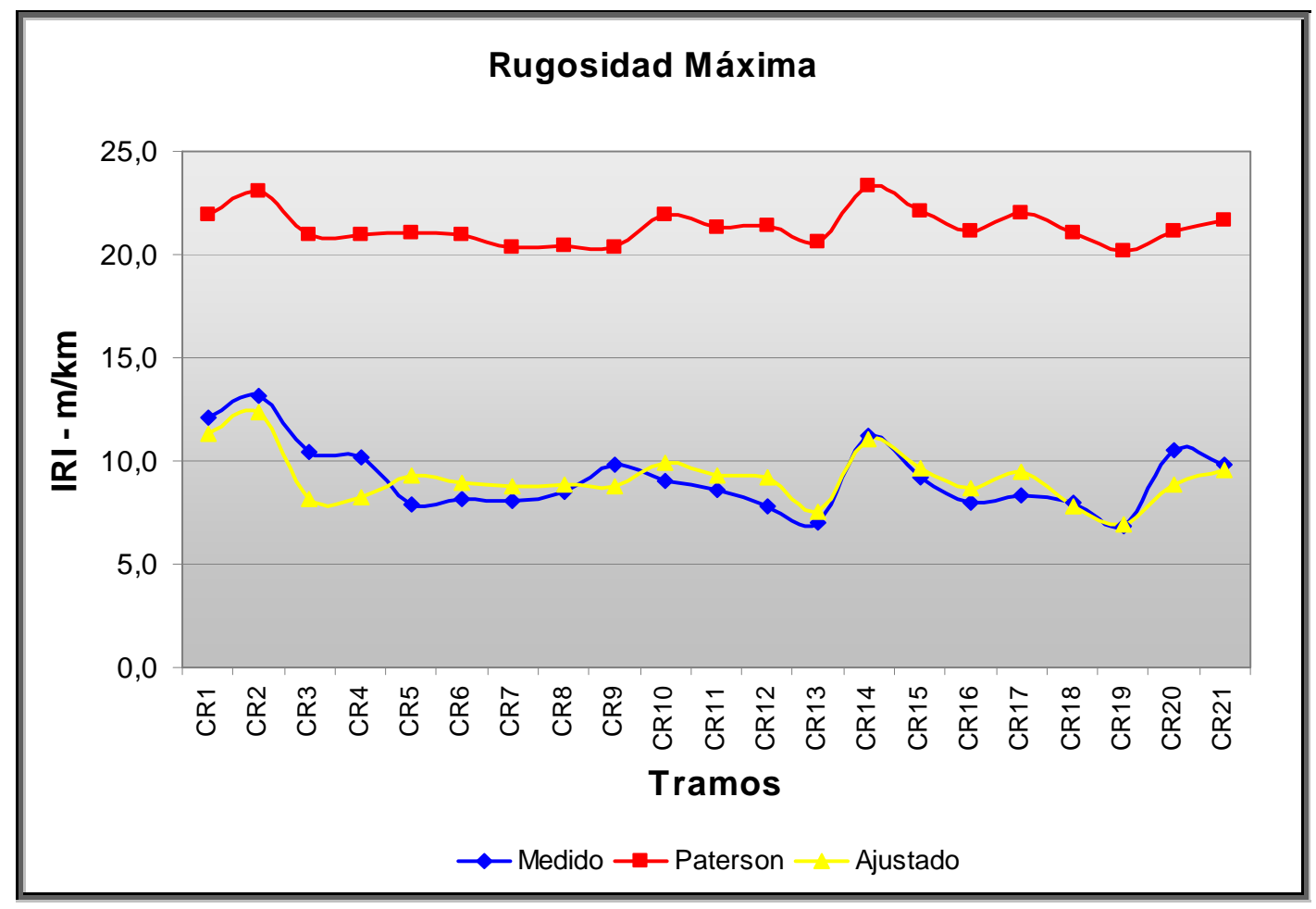

Figura 4.3: Comportamiento de la Rugosidad Máxima

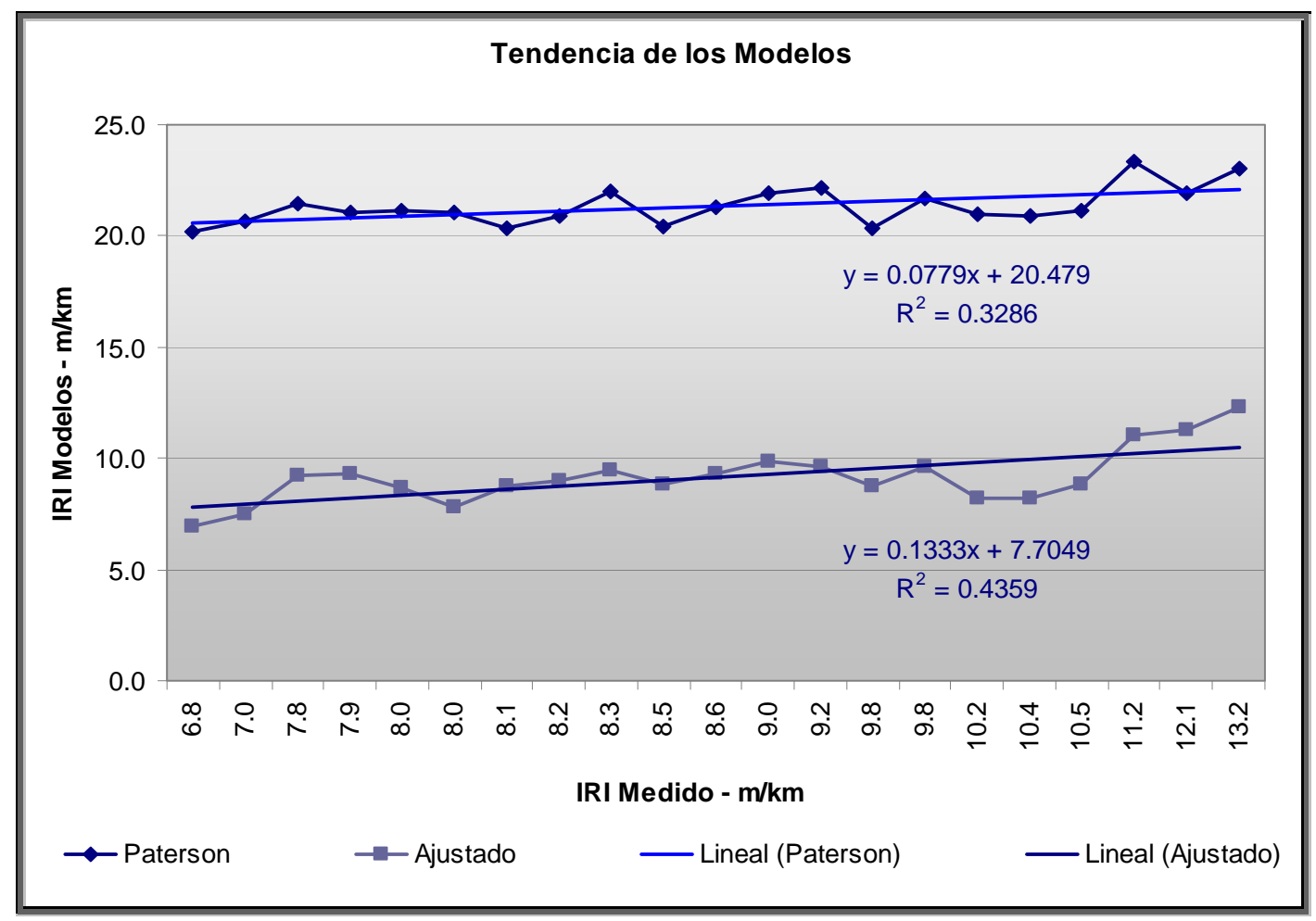

Figura 4.4: Modelo ajustado versus modelo de Paterson

En la figura 4.4 podemos apreciar que la tendencia del modelo ajustado tiene 
mayor pendiente respecto al modelo original de Paterson, indicando por tanto, que se ajustaría mejor a la realidad de las vías de la Región de Antofagasta.

Los resultados anteriores, permiten inferir que los parámetros estimados para Brasil no son útiles para predecir la rugosidad máxima de los caminos no pavimentados de la región de Antofagasta, ya que estos sobrestiman significativamente la rugosidad máxima. Esto justifica la necesidad de ajustar el modelo con datos locales, para obtener predicciones adecuadas de la regularidad de los caminos no pavimentados.

\subsubsection{Ajuste del modelo de Progresión de la Rugosidad}

El modelo de progresión de la rugosidad o variación de la rugosidad en el tiempo, tiene como finalidad predecir la condición superficial del camino en el tiempo, en función de la rugosidad en un tiempo determinado, la rugosidad máxima, el tiempo, el tránsito (vehículos livianos y pesados), la pluviometría y las propiedades de los materiales.

Desde el punto de vista de la ingeniería vial y en el contexto de la región de Antofagasta de Chile, las variables independientes y parámetros que sustentan el modelo de Paterson para Brasil (tránsito liviano y pesado, periodo de medición, rugosidad máxima y rugosidad en un tiempo determinado), se condicen con las características propias de los caminos no pavimentados de la región. Por el contrario, la pluviosidad en la región de Antofagasta es muy baja, lo que hace que el efecto del agua caída sobre el camino sea marginal y que el efecto capturado por está variable sea irrelevante para la realidad regional. Lo que contrasta con las zonas lluviosas donde los caminos no pavimentados pudiesen tener mayor deterioro en los sectores planos, producto de las precipitaciones, y donde las pendientes juegan un rol de protección debido a que evitan la acumulación de agua sobre la superficie del camino, permitiendo así, capturar parte del agua en beneficio de la cohesión del material componente de la capa granular.

El modelo desarrollado en el estudio de Brasil es de forma lineal y se sintetiza en la siguiente ecuación: 


$$
\hat{\mathbf{R I}}_{\mathrm{TG2}}=\mathbf{R} \mathbf{I}_{\max }-\hat{\mathbf{b}} \text { * }\left(\mathbf{R I}_{\max }-\mathbf{R I}_{\mathrm{TG1}}\right)
$$

Donde:

$$
\hat{\mathbf{b}}=\exp \left[\hat{\mathbf{c}}^{*}(\text { TG2 }- \text { TG1) }] \quad \text { En el que: } 0<\mathrm{b}<1\right.
$$

$\hat{c}=-0,001 *[0,461+0,0174 *(A D L)+0,0114 *(A D H)-0,0287 *(A D T) *(M M P / 1000)]$

En tanto que las variables son:

$$
\begin{aligned}
\mathrm{RI}_{\mathrm{TG} 1}= & \text { Rugosidad al tiempo } \mathrm{TG}_{1} \text {, en } \mathrm{m} / \mathrm{km} \mathrm{IRI} \\
\mathrm{RI}_{\mathrm{TG} 2}= & \text { Rugosidad al tiempo } \mathrm{TG}_{2} \text {, en } \mathrm{m} / \mathrm{km} \mathrm{IRI} \\
\mathrm{TG}_{\mathrm{i}}= & \text { Tiempo desde la última nivelación, en días } \\
\mathrm{ADL}= & \text { Tránsito medio diario liviano (bajo } 3.500 \mathrm{Kg} \text { ) en ambas } \\
& \text { direcciones, en vehículos/días } \\
\mathrm{ADH}= & \text { Tránsito medio diario pesado (sobre } 3.500 \mathrm{Kg} \text { ) en ambas } \\
& \text { direcciones, en vehículos/días } \\
\mathrm{ADT}= & \text { Todo el tránsito medio diario (livianos más pesados) en ambas } \\
& \text { direcciones, en vehículos/días }
\end{aligned}
$$

Dada la forma analítica del modelo y los valores medidos en terreno, se buscó ajustar el parámetro "c" de manera recursiva, en función del parámetro "b" y de los datos recolectados en terreno. Para ello se despejo el parámetro "b" de la ecuación de la progresión de la rugosidad, quedando de la forma siguiente:

$$
\mathbf{b}=\left(\mathbf{R I}_{\max }-\mathbf{R I}_{\mathrm{TG2}}\right) /\left(\mathbf{R I}_{\max }-\mathbf{R I}_{\mathrm{TG1}}\right)
$$

Despejando "c" de la expresión original del modelo queda de la forma:

$$
c=\operatorname{Ln} b /\left(T_{2}-T_{G}\right)
$$

De donde podemos obtener "b" y "c" basados en las mediciones realizadas en terreno en distintos períodos de tiempo. 


\section{Ajuste del parámetro "c"}

Por otra parte, se puede estimar el parámetro "c" como una función lineal de un conjunto de variables, cuya expresión es la siguiente:

$$
c=K_{1}+K_{2} * A D L+K_{3} * A D H+K_{4} * A D T * M M P / 1000+e
$$

Siendo "Ki" los parámetros a estimar y "e" es el error aleatorio que representa todas aquellas otras condiciones que afectan las condiciones del camino y que no son capturadas por el modelo.

En el modelo original de Paterson, el tránsito es separado entre tránsito liviano (ADL) y tránsito pesado (ADH). Sin embargo, en el análisis preliminar de los datos, la correlación de estas dos variables estuvo por sobre 95\%, lo que hizo recomendable trabajar con el tránsito total en la estimación, ya que ambas variables contenían la misma información.

Por lo tanto, las variables independientes no presentan correlaciones estadísticamente significativas entre ellas, lo que permite la aplicación del análisis de regresión y avala la estabilidad de los coeficientes estimados del modelo, lo que podemos observar en la matriz de correlación siguiente, donde ninguna resulta estadísticamente distinta de cero (es decir, no existe multicolinealidad significativa).

Tabla 4.28: Correlaciones entre cada variable para 16 muestras

\begin{tabular}{|c|c|c|c|}
\hline \multicolumn{2}{|c|}{ Variables } & $($ ADL+ADH $)$ & ADT*MMP/1000 \\
\hline \multirow{2}{*}{$(\mathrm{ADL}+\mathrm{ADH})$} & Correlación & & $-0,4080$ \\
& p-valor & & 0.1167 \\
\hline \multirow{2}{*}{ ADT*MMP/1000 } & Correlación & $-0,4080$ & \\
& p-valor & 0.1167 & \\
\hline
\end{tabular}

Esto refuerza la idea, por ejemplo, que la variable tránsito liviano más tránsito pesado $(A D L+A D H)$ es independiente de la variable producto tránsito total por pluviometría media mensual (ADT*MMP/1000). 
Los parámetros estimados y sus respectivos estadísticos se presentan en la tabla 4.29.

Tabla 4.29: Variable Dependiente: "c"

\begin{tabular}{|l|c|c|c|c|}
\hline \multicolumn{1}{|c|}{ Parámetro } & Estimación & Error estándar & Estadístico T & P-valor \\
\hline Constante & $-0,00076697$ & 0,000167545 & $-4,57769$ & 0,0005 \\
\hline ADL & $-0,0000080869$ & 0,00000099467 & $-8,13013$ & 0,0000 \\
\hline ADH & $-0,0000080869$ & 0,00000099467 & $-8,13013$ & 0,0000 \\
\hline ADT*MMP/1000 & 0,00361885 & 0,0018859 & 1,9189 & 0,0772 \\
\hline
\end{tabular}

Adicionalmente, en la tabla 4.30 se muestra el análisis de varianza que permite rechazar la hipótesis de que todos los parámetros son iguales a cero, y por lo tanto aseverar que al menos uno de los parámetros sería significativamente distinto de cero. Esto sería congruente con los resultados de la tabla 4.29, que muestra que las dos primeras variables del modelo son estadísticamente significativas al 99\% de confianza, en tanto la última lo es al $90 \%$.

Tabla 4.30: Análisis de la Varianza

\begin{tabular}{|l|c|c|c|c|c|}
\hline \multicolumn{1}{|c|}{ Fuente } & $\begin{array}{c}\text { Suma } \\
\text { cuadrados }\end{array}$ & G.L. & Cuadrado Medio & $\begin{array}{c}\text { Cociente } \\
\text { F }\end{array}$ & P-valor \\
\hline Modelo & 0,000165043 & 2 & 0,00000267335 & 49,49 & 0,0000 \\
\hline Residuo & 0,0000118276 & 13 & 0,000000054016 & & \\
\hline Total (Correl.) & $\mathbf{0 , 0 0 0 0 0 6 0 4 8 9}$ & $\mathbf{1 5}$ & & & \\
\hline
\end{tabular}

El ajuste del modelo para describir la relación entre el parámetro "c" y tres variables independientes, queda de la forma siguiente:

$\hat{c}=-0,001 *(0,76697+0.0080869 * A D L+0.0080869 * A D H-3,61885 * A D T * M M P / 1000)$

El ajuste obtenido tiene una capacidad de explicar el $86 \%$ de las variaciones del parámetro "c" $\left(R^{2}\right.$ ajustado $\left.=0,86\right)$, valor que se puede considerar aceptable, dado que mejora los resultados entregados en estudios anteriores.

La Figura 4.5 denota una varianza homoscedástica, al mostrar valores positivos 
y negativos de los errores estimados, encontrándose la mayoría relativamente cercanos a cero. No evidenciando un comportamiento sistemático de los errores alrededor de su valor esperado o cero, con una distribución uniforme y similar dispersión. Por tanto, la figura evidencia que no existen indicios de heterocedasticidad en los errores de la estimación, lo que es una condición para la aplicación de los modelos de regresión lineal.

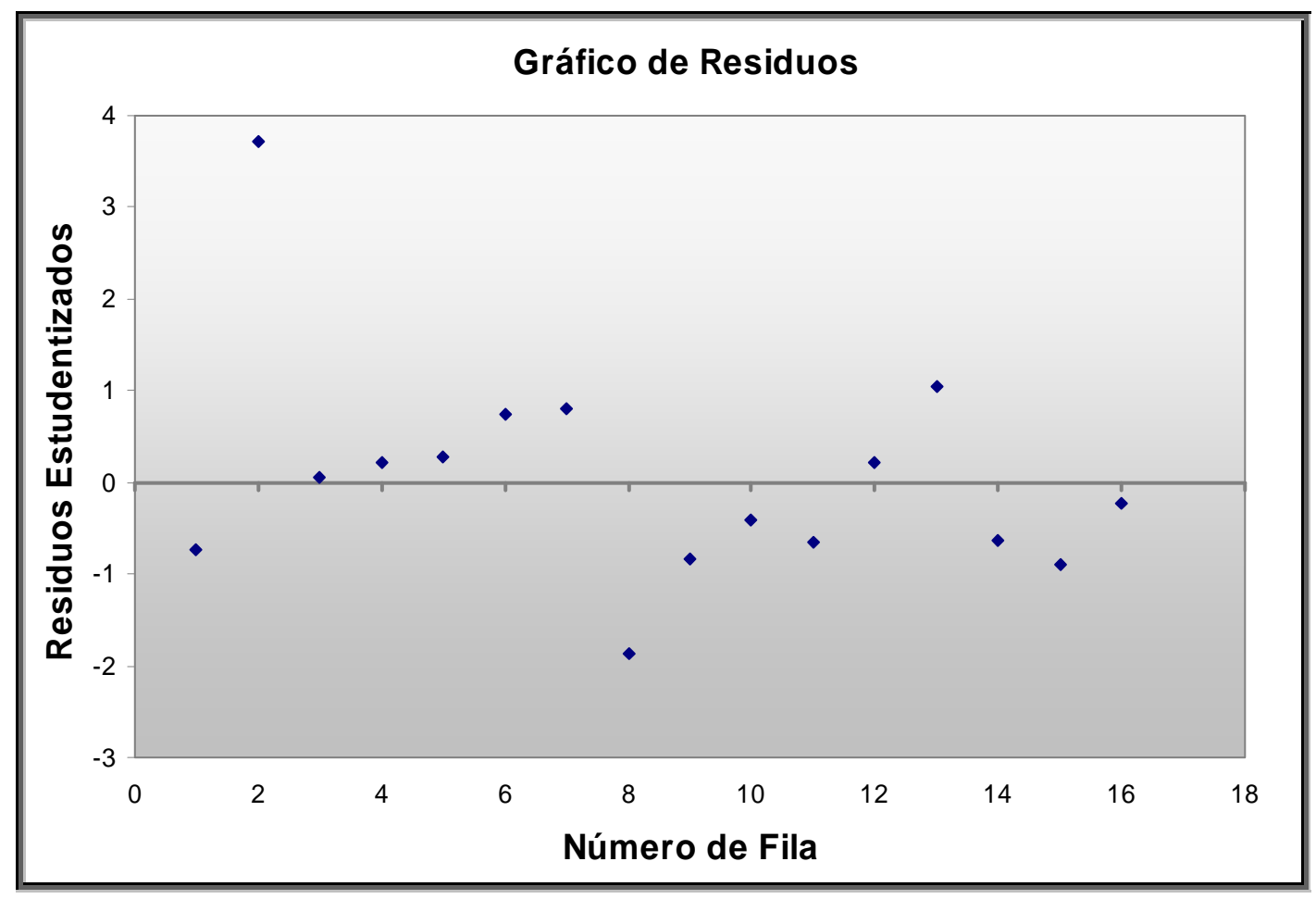

Figura 4.5: Gráfico de residuos - "c"

En atención a lo expuesto, se sugiere adoptar el ajuste propuesto para el parámetro "c", por adaptarse de mejor forma a las condiciones de los caminos no pavimentados de la Región de Antofagasta.

El resumen de los resultados y valores de terreno utilizados en la búsqueda del parámetro "c" se detallan en la tabla 4.31. 
Tabla 4.31: Determinación variable "c"

\begin{tabular}{|c|c|c|c|c|c|c|c|}
\hline \multirow{2}{*}{ Tramo } & \multirow{2}{*}{ MMP } & \multirow{2}{*}{ ADL } & \multirow{2}{*}{ ADH } & \multirow{2}{*}{ ADT } & \multicolumn{3}{|c|}{ Parámetro "c" } \\
\cline { 6 - 8 } & & & & & medido & medido & medido \\
\hline CR1-a & 1,43 & 29 & 33 & 62 & -0.001111 & -0.001111 & -0.001111 \\
\hline CR1-b & 1,43 & 30 & 33 & 63 & -0.000378 & -0.000378 & -0.000378 \\
\hline CR1-c & 1,43 & 31 & 33 & 64 & -0.000940 & -0.000940 & -0.000940 \\
\hline CR2-a & 1,43 & 29 & 33 & 62 & -0.000898 & -0.000898 & -0.000898 \\
\hline CR2-b & 1,43 & 29 & 33 & 62 & -0.000885 & -0.000885 & -0.000885 \\
\hline CR2-c & 0,48 & 31 & 34 & 65 & -0.001014 & -0.001014 & -0.001014 \\
\hline CR7-a & 0,48 & 23 & 4 & 27 & -0.000781 & -0.000781 & -0.000781 \\
\hline CR8-a & 3,40 & 20 & 8 & 28 & -0.001014 & -0.001014 & -0.001014 \\
\hline CR9-a & 3,40 & 20 & 8 & 28 & -0.000828 & -0.000828 & -0.000828 \\
\hline CR7-b & 0,48 & 25 & 8 & 33 & -0.001059 & -0.001059 & -0.001059 \\
\hline CR8-b & 0,48 & 22 & 8 & 30 & -0.001088 & -0.001088 & -0.001088 \\
\hline CR9-b & 2,40 & 22 & 8 & 30 & -0.000700 & -0.000700 & -0.000700 \\
\hline CR16 & 0,14 & 58 & 132 & 190 & -0.001995 & -0.001995 & -0.001995 \\
\hline CR17 & 0,14 & 58 & 132 & 190 & -0.002337 & -0.002337 & -0.002337 \\
\hline CR18 & 0,14 & 58 & 132 & 190 & -0.002389 & -0.002389 & -0.002389 \\
\hline CR19 & 0,14 & 58 & 132 & 190 & -0.002257 & -0.002257 & -0.002257 \\
\hline
\end{tabular}

\section{Cálculo del parámetro "b"}

Con los resultados obtenidos del ajuste del parámetro "c", más los datos medidos en terreno, se calculó el valor de "b", utilizando la forma funcional antes mostrada.

$$
\hat{b}=\exp \left(\hat{c}^{*}\left(T G_{2}-T G_{1}\right)\right)
$$

El parámetro "c" se obtuvo del resultado de su propio ajuste, en tanto que los días que comprende el período de análisis, corresponde a los datos recolectados en el estudio.

El valor calculado para "b" se detalla en la tabla 4.32. 
Tabla 4.32: Valores del parámetro "b"

\begin{tabular}{|c|c|c|c|c|c||}
\hline \multirow{2}{*}{ Tramo } & \multirow{2}{*}{ TG1- TG2 } & \multirow{2}{*}{ "c" Ajustado } & \multicolumn{3}{|c|}{ Parámetro "b" } \\
\cline { 4 - 6 } & & & Medido & Calculado & Paterson \\
\hline CR1-a & 180 & -0.000478 & 0.81867 & 0.77637 & 0.78579 \\
\hline CR1-b & 210 & -0.000465 & 0.92374 & 0.77486 & 0.75210 \\
\hline CR1-c & 420 & -0.000452 & 0.67394 & 0.68711 & 0.56154 \\
\hline CR2-a & 180 & -0.000478 & 0.85076 & 0.77637 & 0.78579 \\
\hline CR2-b & 210 & -0.000478 & 0.83047 & 0.75450 & 0.75484 \\
\hline CR2-c & 420 & -0.000678 & 0.65325 & 0.56585 & 0.55845 \\
\hline CR7-a & 360 & -0.000566 & 0.75490 & 0.65699 & 0.72158 \\
\hline CR8-a & 360 & -0.000325 & 0.69406 & 0.66901 & 0.72391 \\
\hline CR9-a & 360 & -0.000325 & 0.74211 & 0.66901 & 0.72391 \\
\hline CR7-b & 60 & -0.000572 & 0.93846 & 0.87539 & 0.94251 \\
\hline CR8-b & 60 & -0.000602 & 0.93681 & 0.85666 & 0.94547 \\
\hline CR9-b & 60 & -0.000393 & 0.95885 & 0.87750 & 0.94556 \\
\hline CR16 & 60 & -0.001269 & 0.88720 & 0.80621 & 0.83656 \\
\hline CR17 & 60 & -0.001269 & 0.86916 & 0.80621 & 0.83656 \\
\hline CR18 & 60 & -0.001269 & 0.86648 & 0.80621 & 0.83656 \\
\hline CR19 & 60 & -0.001269 & 0.87336 & 0.80621 & 0.83656 \\
\hline
\end{tabular}

\section{Cálculo de la progresión de la rugosidad}

En análisis de regresión de datos de corte transversal en general, la autocorrelación o correlación serial no sería relevante, ya que no existe una dependencia temporal. Por otra parte, en el presente trabajo se realiza una medición que tiene dos puntos en el tiempo para el mismo camino, medición que solo fue usada para calcular la progresión de la rugosidad, que es un solo dato por punto, por lo tanto sigue siendo de corte transversal. De todas maneras, se estimó el índice de Durbin y Watson para medir autocorrelación y este resultó estar muy cercano a dos $(1,96748)$, indicando la no presencia de autocorrelación serial.

Con el ajuste del parámetro "c" y el cálculo del parámetro "b", se procedió a calcular la progresión de la rugosidad. La forma funcional del modelo está dada por: 


$$
\hat{\mathbf{R}}_{\mathrm{TG2}}=\mathbf{R} \mathbf{I}_{\max }-\hat{\mathbf{b}}^{*}\left(\mathbf{R} \mathbf{I}_{\max }-\mathbf{R} \mathbf{I}_{\mathrm{TG1}}\right)
$$

En la tabla 4.33 se detallan los datos utilizados en el cálculo de la progresión de la rugosidad, en ésta podemos observar que la variable estimada (RI $I_{T \text { G2 }}$ ), se encuentra lejos de valores negativos, tanto así, que los valores que se obtienen están dentro del rango esperado para la progresión de la rugosidad (en caminos no pavimentados de la región) y de aquellos valores medidos en terreno.

Tabla 4.33: Cálculo de la variable " $\mathrm{RI}_{\mathrm{TG2}}$ "

\begin{tabular}{|l|c|c|c|c|c|c|c|}
\hline \multirow{2}{*}{ Tramo } & \multirow{2}{*}{$\mathrm{Ri}_{\max }$} & \multicolumn{2}{|c|}{ "b" } & \multirow{2}{*}{$\mathrm{RI}_{\text {TG1 }}$} & \multicolumn{3}{|c|}{ RITG2 } \\
\cline { 8 - 9 } \cline { 7 - 8 } & & Calculado & Paterson & & Medido & Paterson & Calculado \\
\hline CR1-a & 7.3 & 0.84241 & 0.78579 & 2.9 & 3.7 & 3.8 & 3.5 \\
\hline CR1-b & 9.1 & 0.84077 & 0.75210 & 3.7 & 4.1 & 5.0 & 4.5 \\
\hline CR1-c & 12.1 & 0.74556 & 0.56154 & 4.1 & 6.7 & 7.6 & 6.1 \\
\hline CR2-a & 7.8 & 0.84241 & 0.78579 & 3.1 & 3.8 & 4.1 & 3.8 \\
\hline CR2-b & 10.1 & 0.81867 & 0.75484 & 3.8 & 4.8 & 5.3 & 4.9 \\
\hline CR2-c & 13.2 & 0.61398 & 0.55845 & 4.8 & 7.7 & 8.5 & 8.1 \\
\hline CR7-a & 7.9 & 0.71288 & 0.72158 & 3.0 & 4.2 & 4.4 & 4.4 \\
\hline CR8-a & 8.3 & 0.72592 & 0.72391 & 3.4 & 4.9 & 4.8 & 4.7 \\
\hline CR9-a & 9.5 & 0.72592 & 0.72391 & 3.4 & 5.0 & 5.1 & 5.1 \\
\hline CR7-b & 8.1 & 0.94985 & 0.94251 & 4.2 & 4.4 & 4.4 & 4.4 \\
\hline CR8-b & 8.5 & 0.92952 & 0.94547 & 4.9 & 5.1 & 5.1 & 5.2 \\
\hline CR9-b & 9.8 & 0.95214 & 0.94556 & 5.0 & 5.2 & 5.3 & 5.2 \\
\hline CR16 & 8.0 & 0.87479 & 0.83656 & 2.9 & 3.5 & 3.8 & 3.6 \\
\hline CR17 & 8.3 & 0.87479 & 0.83656 & 3.7 & 4.3 & 4.5 & 4.3 \\
\hline CR18 & 8.0 & 0.87479 & 0.83656 & 3.3 & 3.9 & 4.0 & 3.9 \\
\hline CR19 & 6.8 & 0.87479 & 0.83656 & 3.2 & 3.6 & 3.8 & 3.6 \\
\hline
\end{tabular}

Del análisis de la figura 4.6 se aprecia que los modelos son bastante parecidos y aunque aparentemente mantienen la tendencia en la mayoría de los tramos, el modelo de Paterson entrega valores un tanto superiores a los medidos en terreno y a los calculados con el modelo ajustado. Además se aprecia que el modelo ajustado presenta una buena estimación del comportamiento real. 


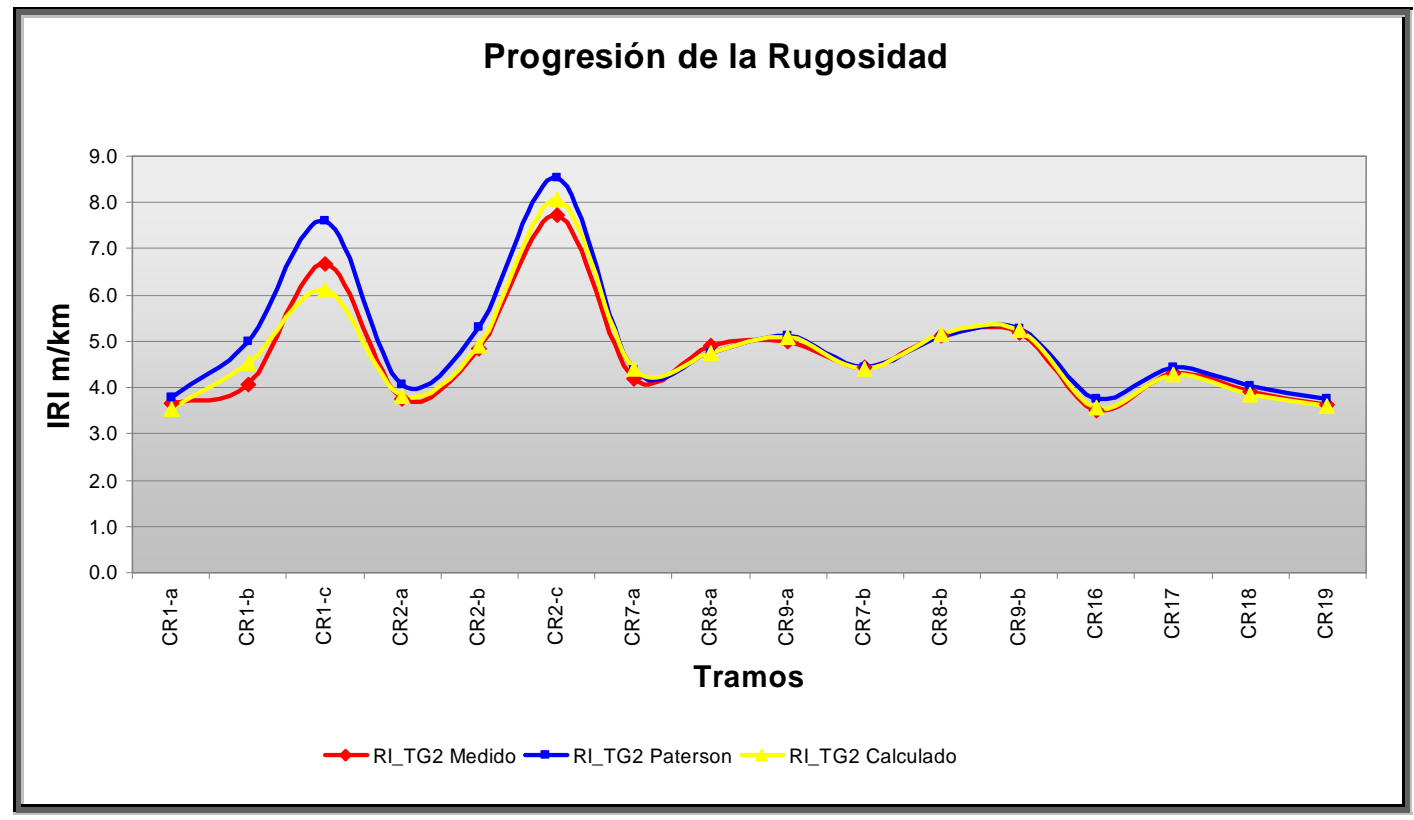

Figura 4.6: Progresión de la Rugosidad

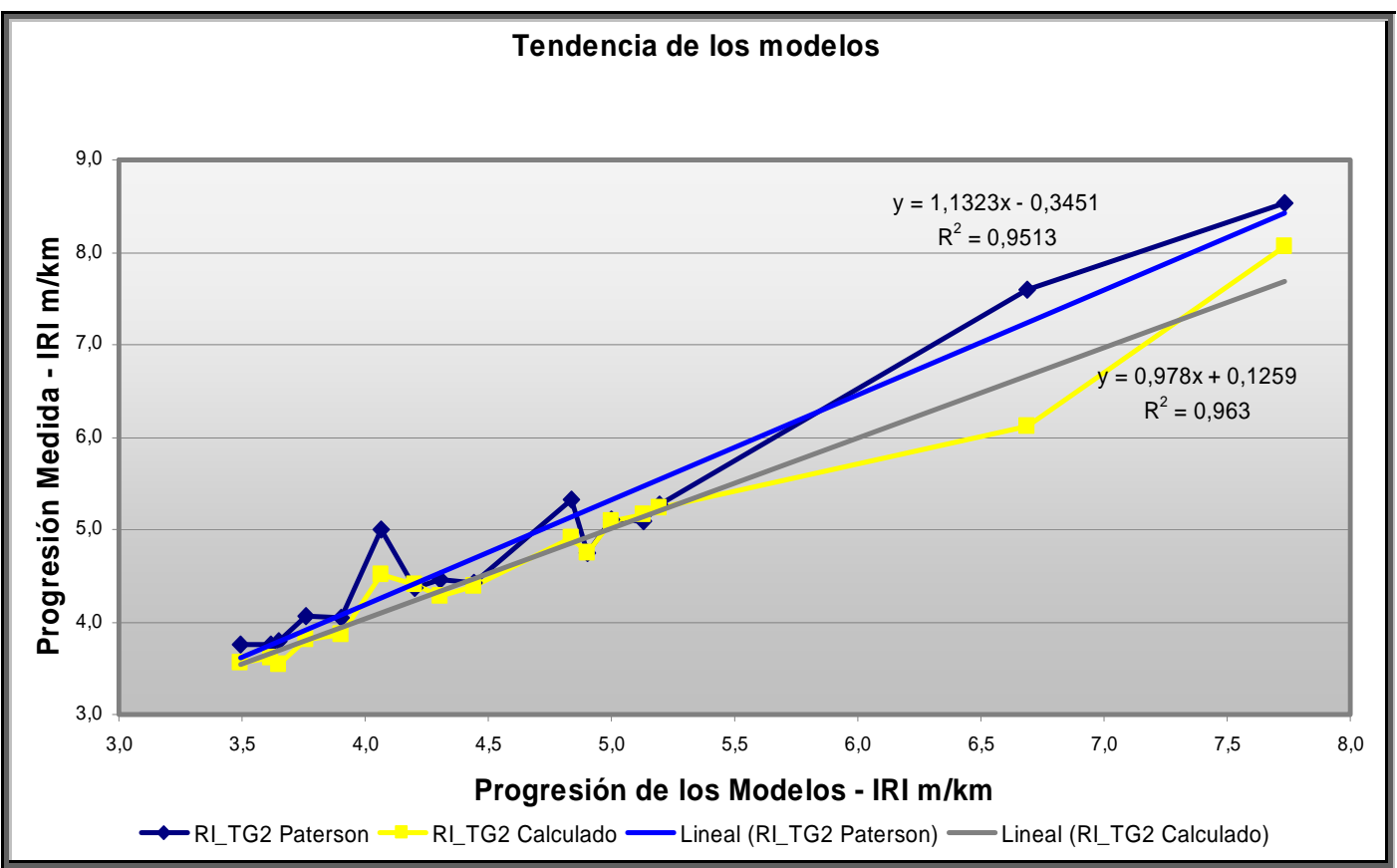

Figura 4.7: Tendencia de los modelos Progresión de RI versus lo medido

En la figura 4.7 podemos apreciar que la tendencia del modelo ajustado tiene una mejor pendiente respecto al modelo de Paterson para Brasil, teniendo un mejor acercamiento a la tangente de $45^{\circ}$, revelando por tanto un ajuste más real a las condiciones y características de los caminos no pavimentados de la Región de Antofagasta. 
De los resultados del estudio, se evidencia que el incremento de la rugosidad no es uniforme, lo que queda de manifiesto al realizar una gráfica rugosidad versus tiempo con los datos de los tramos 1 y 2 , los cuales tienen más de dos periodos de análisis.

De la figura 4.8 se puede concluir que el incremento de la rugosidad en el tiempo, traza un curva de tendencia es exponencial, observándose un mayor incremento a partir del segundo año de servicio de la vía.

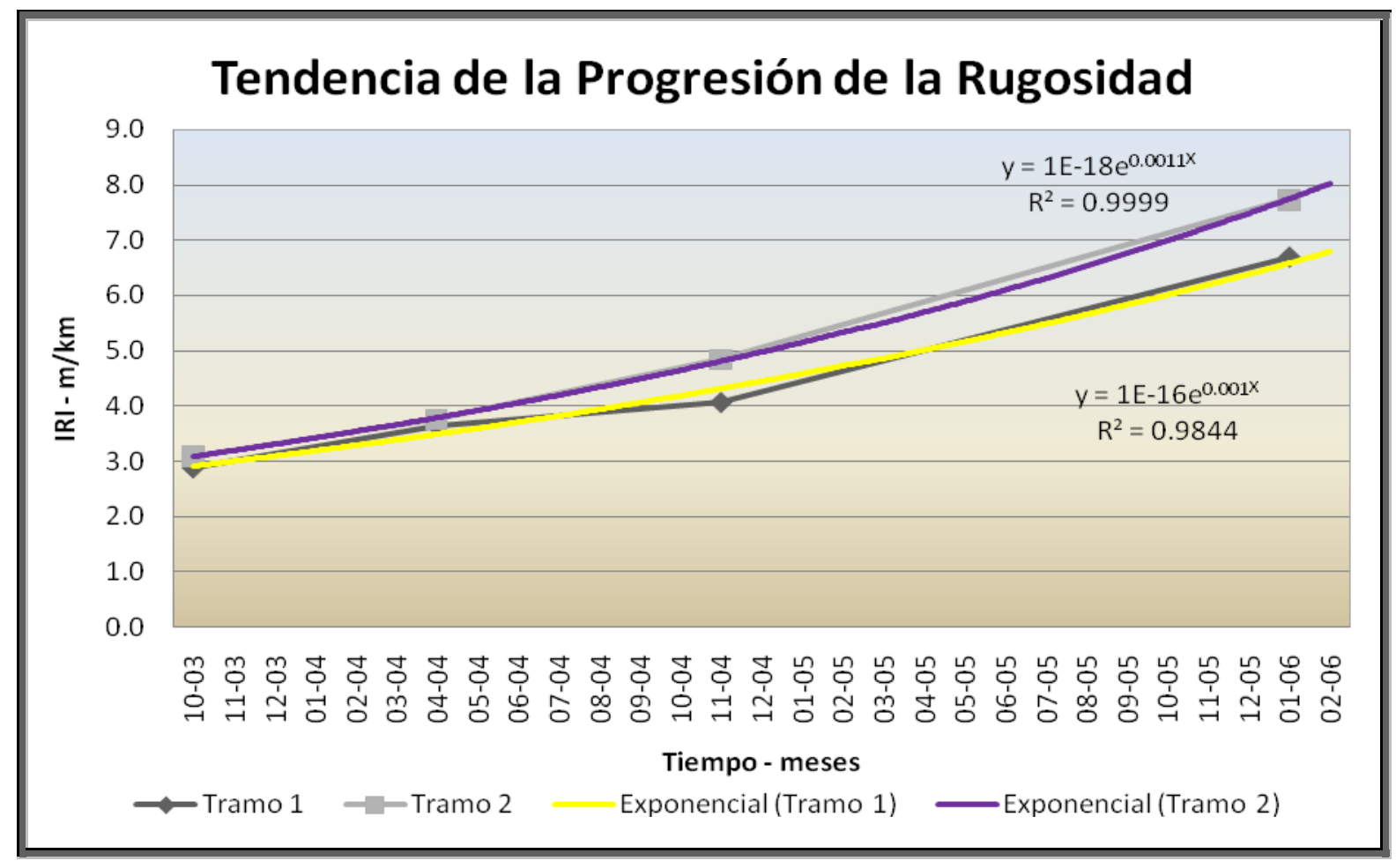

Figura 4.8: Tendencia del incremento de la rugosidad

\subsubsection{Ajuste del modelo de Rugosidad Mínima}

El modelo de la rugosidad mínima de Parteson para Brasil, corresponde a la cota mínima que la rugosidad puede alcanzar bajo ciertas condiciones granulométricas de la carpeta de rodado de los caminos no pavimentados. Específicamente, es función del tamaño máximo de las partículas que pasan el 95\% del material, y de la pendiente de gradación media del material.

Desde el punto de vista de la ingeniería vial, las variables independientes consideradas por Paterson en el estudio de Brasil, para estimar la menor 
rugosidad de los caminos no pavimentados, representan una buena estimación, toda vez que al aumentar el tamaño de las partículas de la gradación del material de las carpetas granulares, sería lógico esperar que aumente la irregularidad superficial de éstas, por tanto la gradación del material y el tamaño máximo de éste, son dos buenos predictores de la rugosidad mínima.

El modelo de Paterson para la rugosidad mínima $\left(R I_{\min }\right)$ se sintetiza en:

$$
\hat{R} I_{\min }=0,36 * D 95 *(1-2,78 * M G)
$$

Donde:

D95 = Tamaño máximo de la partícula del material, definido como la abertura equivalente de la malla a través de la cual pasa el 95\% del material, en $\mathrm{mm}$.

MG = Pendiente de gradación media del material

$$
\text { (MG = mín[MGM, (1-MGM), 0,36] }
$$

La pendiente de gradación media del material es calculada como sigue:

$$
\begin{aligned}
& \text { MGM }=(\text { MG075 }+ \text { MG425 }+ \text { MG02 }) / 3 \\
& M G 075=\operatorname{Ln}(P 075 / 95) / \operatorname{Ln}(0,075 / 95) \quad \text { si: } D 95>0,4
\end{aligned}
$$

En caso contrario: $M G 075=0,3$

$$
\begin{aligned}
\text { MG425 = } & \operatorname{Ln}(P 425 / 95) / \operatorname{Ln}(0,425 / 95) \quad \text { si: } \mathrm{D} 95>1,0 \\
& \text { En caso contrario: MG425 }=0,3 \\
\text { MG02 = } & \operatorname{Ln}(P 02 / 95) / \operatorname{Ln}(2,0 / 95) \quad \text { si: } \mathrm{D} 95>0,4 \\
& \text { En caso contrario: MG02 }=\text { MG425 }
\end{aligned}
$$

Donde : MG075 = corresponde al material que pasa la malla \#200

$$
\text { MG425 = corresponde al material que pasa la malla \#40 }
$$




$$
\text { MG02 = corresponde al material que pasa la malla \#10 }
$$

Utilizando la forma funcional del modelo original, la expresión se puede representar por:

$$
\mathrm{RI}_{\min }=\mathrm{K}_{1}{ }^{*} \mathrm{D} 95-\mathrm{K}_{2}{ }^{*}\left(\mathrm{D} 95^{*} \mathrm{MG}\right)+\mathrm{e}
$$

Siendo $\mathrm{K}_{\mathrm{i}}$ las constantes por determinar $\mathrm{y}$ "e" es el error aleatorio que representa todas aquellas otras condiciones que afectan las condiciones del camino y que no son capturadas por el modelo.

El análisis de correlación de las variables del modelo señala que ésta es alta (mayor al 95\%) entre las variables tamaño máximo de la partícula del material (D95) y la variable producto tamaño máximo de la partícula del material por pendiente de gradación media del material (D95*MG). Por tanto, se modificó la presentación del algoritmo para evitar los efectos de inestabilidad en los parámetros, producto de la multicolinealidad existente. Para definir la nueva ecuación a estimar, se realizó el siguiente desarrollo algebraico:

$$
\begin{gathered}
\hat{\mathrm{R}} \mathbf{I}_{\min }=\mathrm{B}_{1}{ }^{*} \mathrm{D} 95 \text { * }\left(1-\mathrm{B}_{2}{ }^{*} \mathrm{MG}\right) \\
\hat{\mathbf{R}} \mathrm{I}_{\min }=\mathrm{B}_{1}{ }^{*} \mathrm{D} 95-\mathrm{B}_{1}{ }^{*} \mathrm{~B}_{2}{ }^{*} \mathrm{D} 95 \text { *MG } \\
\hat{\mathbf{R}} \mathbf{I}_{\min }=\mathrm{D} 95 \text { * }\left(\mathrm{B}_{1}-\mathrm{B}_{1}{ }^{*} \mathrm{~B}_{2} * \mathrm{MG}\right)
\end{gathered}
$$

Sí : $\quad \mathrm{B}_{1}=\mathrm{K}_{1}$ y $\mathrm{B}_{1}{ }^{*} \mathrm{~B}_{2}=\mathrm{K}_{2}$

Luego, remplazando : $\quad \hat{\mathbf{R I}}_{\min }=\mathbf{D 9 5}{ }^{*}\left(\mathrm{~K}_{1}-\mathrm{K}_{2}{ }^{*} \mathbf{M G}\right)$

Por lo que la forma a estimar sería:

$$
\frac{R I_{\text {mín }}}{D 95}=K_{1}-K_{2} * M G+e
$$

Siendo $\mathrm{K}_{\mathrm{i}}$ las constantes por determinar $\mathrm{y}$ "e" es el error aleatorio que 
representa todas aquellas otras condiciones que afectan las condiciones del camino y que no son capturadas por el modelo.

Los parámetros estimados y sus respectivos estadísticos se presentan en la tabla 4.34.

Tabla 4.34: Variable Dependiente: "RI $I_{\min }$ I D95"

\begin{tabular}{|l|c|c|c|c|}
\hline \multicolumn{1}{|c|}{ Parámetro } & Estimación & Error estándar & Estadístico T & P-valor \\
\hline Constante & 0,190201 & 0,0501554 & 3,79224 & 0,0012 \\
\hline MG & $-0,43025$ & 0,188539 & $-2,28202$ & 0,0342 \\
\hline
\end{tabular}

Adicionalmente, en la tabla 4.35 se muestra el análisis de varianza que permite rechazar la hipótesis de que todos los parámetros son iguales a cero, y por lo tanto aseverar que al menos uno de los parámetros del modelo sería significativamente distinto de cero. Esto sería congruente con los resultados de la tabla 4.34, que muestra que la variable del modelo sería estadísticamente significativa al $97 \%$ de confianza.

Tabla 4.35: Análisis de la Varianza

\begin{tabular}{|l|c|c|c|c|c|}
\hline \multicolumn{1}{|c|}{ Fuente } & $\begin{array}{c}\text { Suma } \\
\text { cuadrados }\end{array}$ & G.L. & Cuadrado Medio & $\begin{array}{c}\text { Cociente } \\
\text { F }\end{array}$ & P-valor \\
\hline Modelo & 0,00455086 & 1 & 0,00455086 & 5,21 & 0,0342 \\
\hline Residuo & 0,0173335 & 19 & 0,000912287 & & \\
\hline Total (Correl.) & $\mathbf{0 , 0 2 2 0 8 4 3}$ & $\mathbf{2 0}$ & & & \\
\hline
\end{tabular}

El ajuste del modelo para describir la relación entre "RI ${ }_{\min }$ / D95" y la variable independiente, queda de la forma siguiente:

$$
\frac{R \hat{I}_{\text {mín }}}{D 95}=0,190201-0,43025 * \text { MG }
$$

Donde $\quad: \quad \mathrm{K}_{1}=\mathrm{B}_{1}=0,190201$

$$
K_{2}=B_{1} * B_{2}=-0,43025
$$


Por tanto : $B_{2}=-0,43025 / B_{1}$

$$
B_{2}=-2,262080641
$$

Así, reformulando el algoritmo de $\mathrm{RI}_{\text {mín }}$, tenemos:

$$
\begin{aligned}
& \hat{R} I_{\min }=B_{1} * D 95 *\left(1-B_{2} * M G\right) \\
& \hat{R} I_{\min }=0,190201 \text { * D95 * }(1-2,262080641 * M G)
\end{aligned}
$$

Este modelo tiene una capacidad de explicar el 17,38\% de las variaciones de la variable dependiente $\left(R^{2}\right.$ ajustado $\left.=0,1738\right)$, lo que sería significativamente mayor al ajuste alcanzado por Paterson para Brasil $\left(R^{2}\right.$ ajustado $\left.=0,11\right)$.

La Figura 4.9 denota una varianza homoscedástica, al mostrar valores positivos y negativos de los errores estimados, encontrándose la mayoría relativamente cercanos a cero. No evidenciando un comportamiento sistemático de los errores alrededor de su valor esperado o cero, con una distribución uniforme y similar dispersión. Por tanto, la figura evidencia que no existen indicios de heterocedasticidad en los errores de estimación del modelo, lo que es una condición para la aplicación de los modelos de regresión lineal.

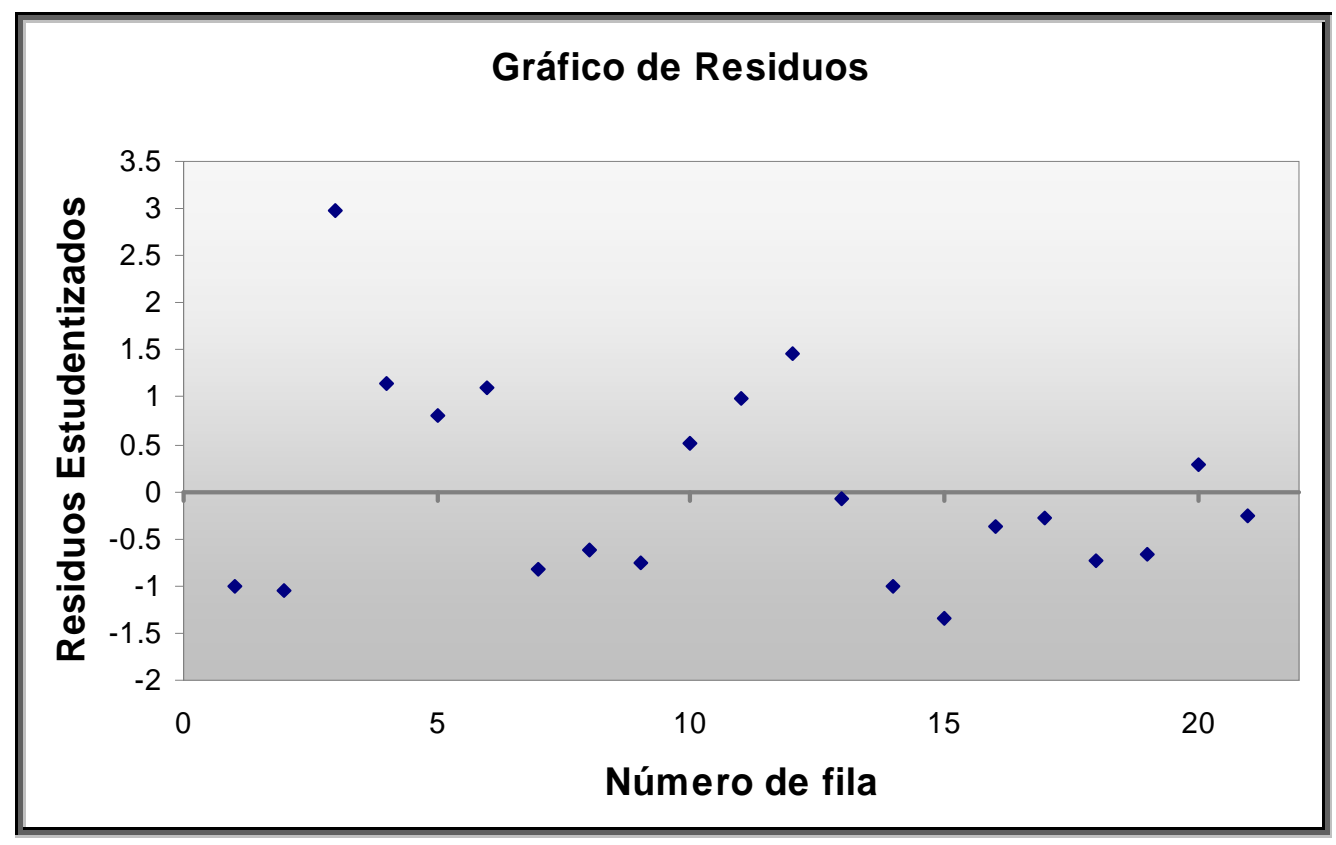

Figura 4.9: Gráfico de residuos - $\mathrm{RI}$ min 
En análisis de regresión de datos de corte transversal en general, la autocorrelación o correlación serial no sería relevante, ya que no existe una dependencia temporal. De todas maneras, se estimó el índice de Durbin y Watson para medir autocorrelación y este resultó ser mayor a dos $(2,28139)$, indicando la no presencia de autocorrelación serial.

En la tabla 4.36 se detallan los datos utilizados en el cálculo de la rugosidad mínima. En la cual podemos observar que la variable controlada $\left(R I_{\text {min }}\right.$ estimada), se encuentra lejos de valores negativos, tanto así, que los valores que se obtienen están dentro del rango esperado para la rugosidad mínima (en caminos no pavimentados de la región) y de aquellos valores levantados en terreno.

Tabla 4.36: Modelo rugosidad mínima

\begin{tabular}{|l|l|l|l|l|l|l|l|l|l|}
\hline \multirow{2}{*}{ Tramo } & \multirow{2}{*}{ MG075 } & MG425 & MG02 & \multirow{2}{*}{ MGM } & MG & \multicolumn{2}{|c|}{ D95 } & \multicolumn{3}{|c|}{ RI $_{\text {min }}$} \\
\cline { 8 - 11 } & & & & & & & Medido & Paterson & Ajustado \\
\hline CR1 & 0,274 & 0,233 & 0,214 & 0,240 & 0,240 & 40 & 2,3 & 4,8 & 3,5 \\
\hline CR2 & 0,274 & 0,214 & 0,195 & 0,227 & 0,227 & 40 & 2,5 & 5,3 & 3,7 \\
\hline CR3 & 0,252 & 0,252 & 0,216 & 0,240 & 0,240 & 25 & 4,0 & 3,0 & 2,2 \\
\hline CR4 & 0,252 & 0,252 & 0,216 & 0,240 & 0,240 & 25 & 3,0 & 3,0 & 2,2 \\
\hline CR5 & 0,330 & 0,238 & 0,231 & 0,266 & 0,266 & 25 & 2,5 & 2,3 & 1,9 \\
\hline CR6 & 0,330 & 0,225 & 0,216 & 0,257 & 0,257 & 25 & 2,8 & 2,6 & 2,0 \\
\hline CR7 & 0,381 & 0,272 & 0,225 & 0,293 & 0,293 & 50 & 2,0 & 3,4 & 3,2 \\
\hline CR8 & 0,381 & 0,272 & 0,225 & 0,293 & 0,293 & 50 & 2,3 & 3,4 & 3,2 \\
\hline CR9 & 0,381 & 0,272 & 0,225 & 0,293 & 0,293 & 50 & 2,1 & 3,4 & 3,2 \\
\hline CR10 & 0,296 & 0,300 & 0,279 & 0,292 & 0,292 & 25 & 2,0 & 1,7 & 1,6 \\
\hline CR11 & 0,286 & 0,283 & 0,262 & 0,277 & 0,277 & 25 & 2,5 & 2,1 & 1,8 \\
\hline CR12 & 0,279 & 0,259 & 0,279 & 0,272 & 0,272 & 20 & 2,3 & 1,7 & 1,5 \\
\hline CR13 & 0,206 & 0,200 & 0,139 & 0,182 & 0,182 & 20 & 2,2 & 3,6 & 2,2 \\
\hline CR14 & 0,240 & 0,220 & 0,182 & 0,214 & 0,214 & 40 & 2,8 & 5,8 & 3,9 \\
\hline CR15 & 0,233 & 0,220 & 0,182 & 0,212 & 0,212 & 40 & 2,5 & 5,9 & 4,0 \\
\hline CR16 & 0,381 & 0,307 & 0,261 & 0,316 & 0,316 & 50 & 2,2 & 2,2 & 2,7 \\
\hline CR17 & 0,381 & 0,307 & 0,261 & 0,316 & 0,316 & 50 & 2,3 & 2,2 & 2,7 \\
\hline CR18 & 0,381 & 0,264 & 0,225 & 0,290 & 0,290 & 50 & 2,2 & 3,5 & 3,3 \\
\hline CR19 & 0,381 & 0,264 & 0,225 & 0,290 & 0,290 & 50 & 2,3 & 3,5 & 3,3 \\
\hline CR20 & 0,318 & 0,242 & 0,269 & 0,276 & 0,276 & 50 & 4,0 & 4,2 & 3,6 \\
\hline CR21 & 0,274 & 0,242 & 0,239 & 0,252 & 0,252 & 50 & 3,7 & 5,4 & 4,1 \\
\hline
\end{tabular}


Del análisis de la figura 4.10 se aprecia que tanto el modelo de Paterson para Brasil, como el modelo ajustado, tienden a sobre estimar la rugosidad mínima. Sin embargo, en aquellos sectores en que el modelo ajustado subestima la rugosidad mínima, este fenómeno puede deberse a que las mediciones fueron realizadas posterior a la puesta en servicio de las vías.

Además, es de recordar que los modelos estiman la rugosidad mínima en función de las características de los materiales (tamaño máximo y gradación granulométrica), no incluyendo la variable tránsito, la cual tendería a elevar el valor de la rugosidad mínima.

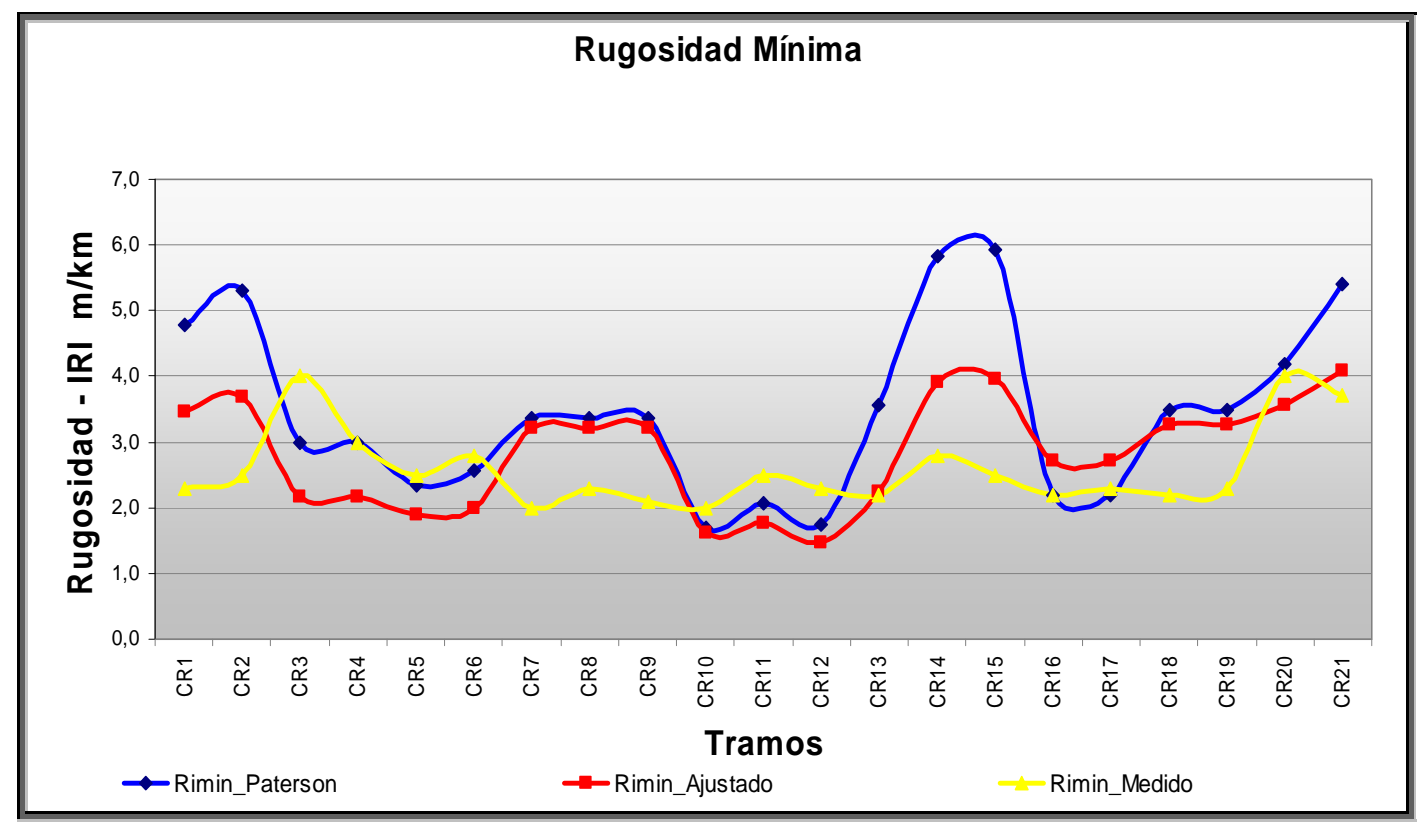

Figura 4.10: Rugosidad Mínima

En la figura 4.11 se apreciar que el modelo de Paterson para Brasil versus el modelo ajustado, sobreestima la rugosidad mínima para igual valor de rugosidad medida en terreno.

Las pendientes para ambos modelos son bajas, lo que reafirma el valor de $R^{2}$ ajustado $(0,1738)$ encontrado para la regresión lineal del modelo ajustado. 


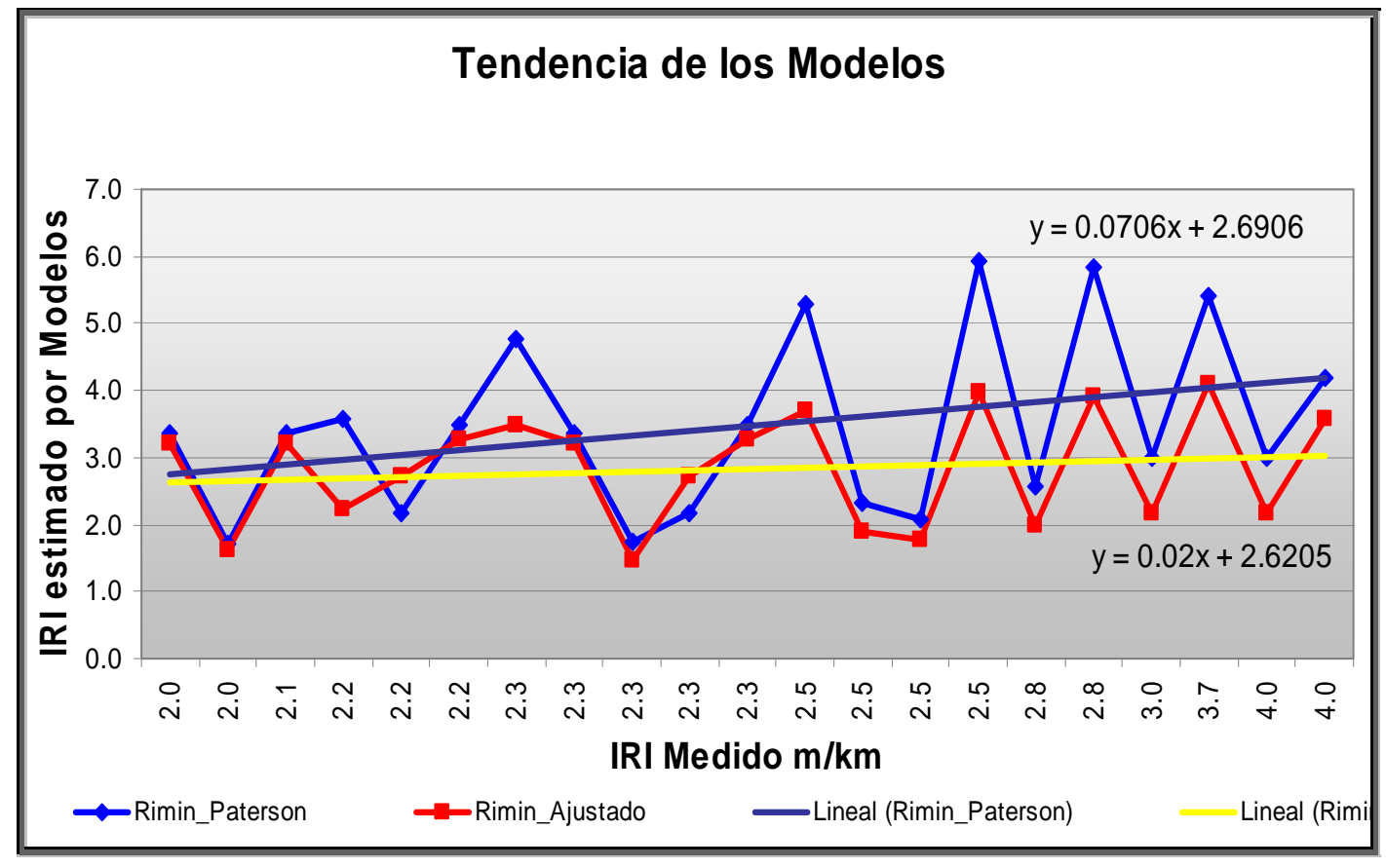

Figura 4.11: Modelo $\mathrm{RI}_{\min }$ ajustado versus modelo de Paterson

Finalmente, se deduce de lo expuesto que el modelo ajustado tiene un mejor acercamiento a los valores reales de rugosidad mínima, bajo las características propias de la Región desértica de Antofagasta y sus caminos no pavimentados.

\subsubsection{Ajuste del modelo de pérdida de material}

El recebo es la operación más importante del mantenimiento de un camino no pavimentado, equivalente a la importancia de recubrir un camino pavimentado, su frecuencia requerida es una decisión importante de la planificación.

La pérdida de material se define como el cambio de espesor de la carpeta de grava durante un período de tiempo y se usa para estimar cuando el espesor de la carpeta granular ha disminuido a un nivel dónde el recebo es necesario.

Paterson identificó tres factores importantes que influyen en la pérdida de material de la carpeta granular: el tráfico, las propiedades del material (material fino e índice de plasticidad) y la geometría del camino (grado de curvatura y pendiente). La expresión del modelo de Paterson para Brasil, contempla dentro de sus principales variables independientes, la precipitación mensual, el volumen de tránsito, la geometría del camino y las características geotécnicas 
de la carpeta granular.

Este modelo no fue ajustado por las siguientes razones:

- Una de los motivos más importantes, es que la Dirección de Vialidad de Chile no tiene contemplado operaciones de recebo en los caminos no pavimentados del programa "Caminos Básico 5000". Programa que consiste en el mejoramiento de cinco mil kilómetros de caminos con carpetas granulares estabilizadas con diferentes materiales, dependiendo la región del país. Que para el caso de la región de Antofagasta, estos fueron estabilizados con bischofita.

- Otro factor que no permitió ajustar el modelo fue la falta de una base de dato histórica (marco estadístico) que permitiera estimar el modelo.

- No existe un método confiable para levantar datos de terreno. Las formas originales de medir la pérdida de material es a través de calicatas o por un levantamiento topográfico. Métodos de considerable aleatoriedad, lo que conlleva a sumar errores importantes por medición.

- Sin embargo, se realizaron mediciones en algunos caminos comprendidos en el presente estudio y que se insertan en el programa "Caminos Básico 5000", para desarrollar una estimación del modelo, las que resultaron infructuosas ya que la gran cantidad de variables independientes del modelo no permite realizar regresiones con muestras pequeñas.

En definitiva, este modelo no se ajusto por las razones expuesta y por tanto debiera ser considerado en un próximo estudio. Es recomendable en primer lugar, diseñar un método científico más confiable para realizar las mediciones, y en segundo lugar, levantar una cantidad de datos en directa relación con el número de variables independiente, que en definitiva definen los grados de libertad del análisis estadístico del modelo. 


\section{CAPITULO V}

\section{CONCLUSIONES}

\subsection{CONCLUSIONES GENERALES}

En adelante se describen las conclusiones que derivan del presente estudio "Ajuste de las variables que gobiernan los modelos de comportamiento del HDM-4 para vías no pavimentadas de la región de Antofagasta - Chile".

Respecto a los modelos de rugosidad, se ajustaron los parámetros de las variables propuestas por Paterson para Brasil. Los valores encontrados para la región de Antofagasta de Chile, se obtuvieron con datos recopilados en seguimientos realizados por la Dirección Nacional de Vialidad y de aquellos levantados en terreno durante el presente estudio, en caminos estabilizados con bischofita.

Las principales conclusiones a las cuales se arribaron en el estudio se resumen a continuación:

\subsubsection{Modelos de rugosidad}

La calidad del ajuste realizado es estadísticamente significativa y con un poder explicatorio superior a los conseguidos por los modelos de Paterson para Brasil, actualmente en uso.

Es importante mencionar que los mecanismos de toma de muestras, el personal encargado de las mediciones y el contexto ambiental, no repercuten en diferencias sustantivas en los valores medidos en terreno.

Sin embargo, una tecnología superior de medición a las actuales, bien podría suponer que las medidas históricas cuenten con un grado más grande de error, haciendo que la capacidad de explicación que tiene la variable se pierda por problemas métricos.

Los parámetros propuestos para estimar la rugosidad máxima, mínima y su progresión, para las condiciones y características de la región de Antofagasta, 
son el resultado de un detallado análisis estadístico, por tanto son un aporte a utilizar en las estimaciones futuras para la planificación de la red de caminos básicos de la Región de Antofagasta. Estos resultados, que son una mejora al modelo de Paterson actualmente utilizado para estos efectos, deberían ser periódicamente validados con nuevas tramificaciones, para capturar los efectos de cambios en las condiciones de los caminos como de los vehículos que los utilizan.

Es necesario recordar, que por la naturaleza de los deterioros a cuantificar, ni los modelos desarrollados para Brasil y países africanos, han alcanzado niveles altos de explicación de la variabilidad en las condiciones de los caminos, sin embargo, la fortaleza de esos resultados radica en que se han obtenido para un considerable número de tramificaciones, lo que hace que su validez sea robusta.

Por otro lado, es preciso mencionar, que si bien los parámetros de los modelos fueron ajustados a la realidad regional, la forma funcional de éstos no fue variada, condición sinecuanum del programa HDM-4.

También es importante recordar y destacar, que no obstante se hayan encontrados los valores de ajustes de las variables de los modelos, el software del HDM-4 no permite que estos valores sean modificados, admitiendo solamente ingresar los nuevos umbrales de rugosidad obtenidos. Los modelos ajustados pueden ser utilizados de forma independiente y externa, en planillas electrónicas, del tipo Excel de Microsoft Office.

Es interesante subrayar, que los modelos implementados en HDM-4 son altamente sensibles a las características granulométricas, razón explicativa de que el modelo no entregue mejores resultados. Esta característica de los modelos, propia de su diseño, tiende a limitar las predicciones en el largo plazo, si se considera que, las variables que incluyen las características granulométricas de las capas granulares, son variables en el tiempo por el efecto producido por el tránsito y las lluvias, las que modifican en forma significativa el contenido de material fino existente en la capa granular de 
rodado. Esta es una justificación adicional de la necesidad de reestimar los modelos periódicamente.

Uno de los resultados más importantes del estudio, corresponde al hecho de conseguir establecer relaciones tanto para la rugosidad máxima, como para la mínima. Lo que representa una ventaja respecto al hecho de considerar la rugosidad máxima, como un valor fijo y ostensiblemente alto, ya que estas variables tienen directa relación en los resultados del análisis técnico económico que entrega el HDM-4.

Respecto de la progresión de la rugosidad, el modelo ajustado presenta una buena estimación del comportamiento real, versus el modelo de Brasil, con valores más cercanos a los medidos en terreno. Además se evidencia que la tendencia del incremento de la rugosidad es exponencial, observándose un mayor incremento a partir del segundo año de servicio de la vía.

Los valores estimados por las expresiones obtenidas de los modelos ajustados, se alejan de valores cero o negativos, es más, los valores que se obtienen están dentro del rango esperado para el comportamiento estimado para caminos no pavimentados de la región.

Respectos al Modelo de rugosidad antes y después de un reperfilado no fue ajustado, ya que las vías consideradas en el estudio son aquellas que corresponde al programa de gobierno "Caminos Básicos 5000", rutas que fueron diseñadas siguiendo la metodología AASHTO, con horizontes de vida sobre los tres años con estrategias de conservación cero. En consecuencia, no es posible medir la variable rugosidad antes y después del reperfilado.

En relación con el Modelo de Rugosidad en estaciones climáticas, el capítulo tres (sección 3.7.3) explica la particularidad pluviométrica de la Región de Antofagasta, la que geográficamente se ubica en el Desierto de Atacama, el más árido del mundo. Esta singularidad y la errática presencia de lluvias, no permite tener registros de rugosidad asociada a pluviometría en la región. No teniendo sentido ajustar este modelo para una zona completamente seca. 


\subsubsection{Modelo pérdida de material}

El recebo es la operación más importante del mantenimiento de un camino no pavimentado, equivalente a la importancia de recubrir un camino pavimentado, su frecuencia requerida es una decisión importante de la planificación.

Recordar que la pérdida de material se define como el cambio de espesor de la carpeta de grava durante un período de tiempo y se usa para estimar, cuando el espesor de la carpeta granular ha disminuido a un nivel precario, dónde el recebo es necesario. El que se ha estimado en función del tráfico, las propiedades del material (material fino e índice de plasticidad) y la geometría del camino (grado de curvatura y pendiente).

La estimación del cambio de espesor en terreno, es la condición más precaria en que se encuentra el modelo de Pérdida de Material, lo que no admite obtener una medición confiable que permita desarrollar un buen estimador.

Además de lo expuesto, otras razones que contribuyeron a que el modelo no se ajustara son las siguientes:

- LA Dirección de Vialidad de Chile no tiene contemplado operaciones de recebo en los caminos no pavimentados del programa "Caminos Básico 5000", caminos bases del presente estudio.

- No existe una base de dato histórica (marco estadístico) que permita comparar mediciones y realizar regresiones para estimar el modelo.

- No existe un método confiable para levantar datos de terreno. Las formas originales de medir la pérdida de material es a través de calicatas o por un levantamiento topográfico. Métodos de considerable aleatoriedad, lo que conlleva a sumar errores importantes por medición.

En definitiva, este modelo debiera ser considerado en un próximo estudio, mejorando las condiciones de medición y el número de tramos testigos, los que debieran estar en una relación 1:10 respecto del número de variables independientes del modelo. 
Respecto al modelo de Pérdida de Material brasileño desarrollado por Paterson e implementado en HDM-4, es claro que su uso directo no es apropiado para la realidad chilena, por cuanto la forma funcional de la ecuación no permite que el ajuste del modelo pase simplemente por realizar reajustes del mismo, intentando encontrar parámetros que modifiquen la función, para que entregue valores más apropiados. Lo anterior se deduce debido a que los rangos de precipitación, principalmente, hacen que para la realidad brasileña, extrapolaciones sobre valores superiores de agua caída, el modelo continúe moviéndose en una relativa directa proporcionalidad, no siendo esto válido para valores bajos de precipitación, invirtiéndose la proporción para estos niveles.

Además, es importante consignar que los caminos chilenos poseen porcentajes inferiores al 55\% de material fino, por lo que el modelo predice pérdidas que no se ajustan a la realidad.

En razón a lo expuesto, mientras no se desarrolle una nueva formulación teórica para cuantificar la Pérdida de Material para las condiciones regionales, es recomendable continuar utilizando el modelo original de Paterson para proyectar la Pérdida de Material, aun cuando ésta arroje valores que distan de los medidos en terreno.

\subsection{RECOMENDACIONES}

Respecto a los modelos de rugosidad, las nuevas expresiones encontradas se acercan bastante más a la experiencia empírica existente, por lo cual parece ser un resultado más cercano a nuestra realidad. A pesar de ello, es recomendable que estos modelos se asuman como un avance en la búsqueda de expresiones más realistas y certeras, en ningún caso como un resultado definitivo, ya que siempre será necesario efectuar nuevas mediciones relativas a aumentar las bases de datos y así permitir la validación y mejoramiento de las expresiones aquí encontradas.

Por otro lado, los modelos desarrollados pueden describir en forma satisfactoria el fenómeno dentro del área de estudio, siendo su generalización un tanto cuestionable, motivo más que necesario para ampliar la zona testigo para 
conocer la realidad en otras regiones del país, que con condiciones geográfico ambientales diferentes, pudiesen presentar comportamientos diferentes a los modelables por las expresiones encontradas.

Se recomienda estudiar el efecto del drenaje en la rugosidad y la pérdida de material de estos caminos, que de acuerdo con lo observado se comporta inversamente proporcional a los modelos, es decir, cuanto mejor sea el drenaje del camino menor será la rugosidad media del camino.

Por otra parte, las Direcciones Regionales de Vialidad no poseen en la actualidad equipos para la cuantificación objetiva de la pérdida de material, siendo recomendable diseñar un método científico más confiable para realizar dichas mediciones.

Se recomienda que futuras investigaciones de los modelos de deterioro de caminos no pavimentados, incorporen o desarrollen modelos particulares para describir deterioros específicos y de mayor importancia en los caminos nacionales, como lo son las "calaminas"1 y la generación de "baches", puntualizando de esta manera aún más los problemas de las actuales capas granulares, derivando así en caminos más estables y duraderos.

\footnotetext{
${ }^{1}$ Dirección de Vialidad - MOP - (2000). Manual de Carreteras, Volumen № 7 "Mantenimiento Vial"
} 


\section{GLOSARIO DE TÉRMINOS}

Calamina: Ondulaciones transversales de la superficie del camino que tiene su origen en un movimiento plástico de los suelos que la conforman, causadas por las ruedas de los vehículos. El fenómeno puede deberse a varias razones pero, con mayor frecuencia, se produce en capas conformadas por partículas de tamaño relativamente grande ligadas con un suelo fino y $\sin$ o con pocos tamaños intermedios, es decir, suelos que tienen una granulometría discontinua.

Las ondulaciones o calamina son habitualmente normales al eje del camino y se presentan a intervalos más o menos regulares; originan superficies de rodadura extremadamente rugosas y dan una mala estabilidad direccional a los vehículos. Se clasifican en ondulaciones "sueltas" y "fijas"; las primeras consisten en crestas paralelas de material fino arenoso suelto, en tanto que las fijas tienen crestas duras y paralelas de material fino arenoso compactado.

Bache: Hoyos de diversos tamaños que se producen en la superficie de rodadura por desintegración local.

Calicata: Exploración que se hace en cimentaciones de edificios, muros, caminos, etc., para determinar, identificar y clasificar los materiales constituyentes de los suelos de fundación, a través de estratigrafía y ensayes.

IRI: Índice de Rugosidad Internacional. Este índice mide la rugosidad de un pavimento, es decir, la irregularidad superficial de una capa de rodadura. Es el parámetro de estado más característico de la condición funcional de ésta y el que incide directamente en los costos de operación de los vehículos. 
ISOYETAS: Conocidas también como isohietas, son líneas imaginarias que unen puntos de igual magnitud de precipitación media mensual. Sirven para conocer la distribución geográfica de la precipitación en una región, país, continente; además permiten delimitar zonas de aridez y de exceso de lluvias.

\section{GLOSARIO SIGLAS}

AASHO : Asociación Americana de Funcionarios de Carreteras Estatales (American Association of State Highway Officials).

ADH : Tránsito pesado (veh./día)

ADL : Tránsito liviano (veh./día), (NC según el estudio de Brasil)

ADT : Tránsito medio diario anual (veh./día), (NC según el estudio de Brasil)

AIPCR : Asociación mundial de Carreteras (Association mondiale de la route). Capítulo francés.

D95 : Tamaño máximo del agregado $(\mathrm{mm})$

HDM : Desarrollo de Modelos de Gerenciamiento de Carretera (Highway Design and Maintenance Standards Model)

IDIEM : Centro de Investigación, Desarrollo e Innovación de Estructuras y Materiales

IRI : Índice de rugosidad internacional

ISOHDM : Estudio Internacional de Herramientas para el Desarrollo y Administración de Carreteras (International Study of Highway 
Development and Management Tools)

KCV : Curvatura horizontal (grados/kilómetro)

$\mathrm{Ki} \quad$ : Constante de ajuste del HDM

KT : Coeficiente del material removido por el tránsito inducido, expresado como una función de la precipitación, geometría del camino y características del material.

MG : Pendiente de gradación media del material

MGD : Relación del material en gradación polvo

MIT : Instituto Massachussets de Tecnología

MLA : Pérdida de Material anual en mm/año.

MMP : Precipitación media mensual (metros/mes)

MOP : Ministerio de Obras Públicas de Chile

P075 : Porcentaje de material que pasa malla № 200

PI : Índice de Plasticidad

PIARC : World Road Association (Asociación Mundial de Carreteras). Capítulo de los Estados Unidos.

PNUD : Programa de Naciones Unidas para el Desarrollo

QI : Rugosidad (IRI)

RF : Promedio subidas más bajadas del camino $(\mathrm{m} / \mathrm{km})$

RTIM : Modelos de Inversión del Transporte Caminero 
$t_{1}, t_{2} \quad:$ Tiempo en días

TRRL : : Transport and Road Research Laboratory

VOC : Costos de operación de vehículos

WMO : Organización Mundial de Meteorológica (World Meteorological Organization) 


\section{REFERENCIAS}

1. AASHO (1952). Road User Benefit Analyses for Highway Improvements. American. Association of State Highway Officials, Washington, D.C.

2. Araya C., Lorena (1988). "Validación del Modelo de Deterioro Brasileño de Caminos no Pavimentados. Aplicación al caso Chileno".

3. Bennett, C R. and Greenwood, I.D. (2005). Volume 7. Modeling Road User and Environmental Effects in HDM-4. The Highway Development and Management Series.

4. Chesher, A.D. and Harrison, R. (1987). Vehicle Operating Costs: Evidence from Developing Countries. World Bank Publications, Johns Hopkins Press.

5. Christopher R. Bennett \& William D. O. Paterson. (2000). Volume 5 del HDM-4 Guide to Calibration and Adaptation of HDM-4.

6. De Solminihac H.; Hidalgo P.; Salgado M. (2001), Seguimiento para la calibración de Modelos de Deterioro de Pavimentos Asfálticos. Ministerio de Obras Públicas y Pontificia Universidad Católica de Chile.

7. Dirección de Vialidad - MOP - (2000). Manual de Carreteras, Volumen No 7 "Mantenimiento Vial"

8. Dirección Nacional de Vialidad, Chile. (2005). Página Web oficial: http://www.vialidad.gov.cl

9. García A., José (1993). "Estudio del Comportamiento de Caminos no Pavimentados de la Zona Central de Chile".

10. Hide, H., Abaynayaka, S.W., Sayer, I., Wyatt, R.J. (1975). The Kenya Road Transport Cost Study: Research on Vehicle Operating Costs. Transport and Road Research Laboratory Report LR672, Department of the Environment, Crowthorne.

11. Hodges, J.W., Rolt, J. and Jones, T.E. (1975). The Kenya Road Transport Cost Study: Research on Road Deterioration. TRL Report LR 673, Department of the Environment, Crowthorne.

12. IDIEM - Vialidad (2000). Informe final "Análisis de los Modelos de Deterioro para Caminos no Pavimentados Incluidos en el HDM-III y Proposición de Umbrales de rugosidad para Evaluaciones Económicas"

13. Inzunza B. Juan Carlos. "Climas de Chile. Capitulo 15 ". Universidad de Concepción de Chile. http://www2.udec.cl/ jinzunza/meteo/meteo.htm

14. J. B. Odoki \& Henry G. R. Kerali (2001). Volume 4. Analytical Framework and Model Descriptions 
15. Kerali, H.; Robinson, R.; Paterson, W. (1996), New Highway Development and Management Tools (HDM-4). Seventy Fifth Anniversary Annual Meeting. Transportation Research Board and National Research Council. Washington D.C.

16. MOP. (Ministerio de Obras Públicas) (2005). Dirección de Vialidad. Subdirección de Planificación y Estudios. Informe anual "Red Vial Nacional Dimensionamiento y Características".

17. PIARC. (2005). Página Web oficial de la "World Road Association" (Asociación Mundial de Carreteras). http://www.piarc.org/es/

18. Robinson, R., Hide, H., Hodges, J.W., Rolt, J. and Abaynayaka, S.W. (1975). A Road Transport Investment Model for Developing Countries. TRL Report LR 675, Department of the Environment, Crowthorne.

19. Videla, C.; De Solminihac, H.; Gaete, R.; Bustos, M. (1996), Ajuste de Factores de Calibración para Ampliar Modelos de Deterioro de Pavimentos Asfálticos. Ministerio de Obras Públicas y Pontificia Universidad Católica de Chile.

20. Watanatada T, Harral C G, Paterson W D O, Dhareshwar A M, Bhandari A, and Tsunokawa K, (1987), The Highway Design and Maintenance Standards Model, Volume 1 - Description, The World Bank, John Hopkins University Press.

21. Watanatada, T. (1981). Highway Design and Maintenance Standards Model (HDM) Model Description and User's Manual - Release II. Transportation, Water and Telecommunications Department Report, the World Bank, Washington, D.C.

22. William Paterson D.O. (1987). "Road Deterioration and Maintenance Effects: Models for Planning and Management. Highway Desing and Maintenance Standards Series", World Bank Transportation department, Washington D.C. 


\section{ANEXOS}




\section{ANEXO 1: Fotográfico}

\section{Caminos tipos considerados en el presente estudio}

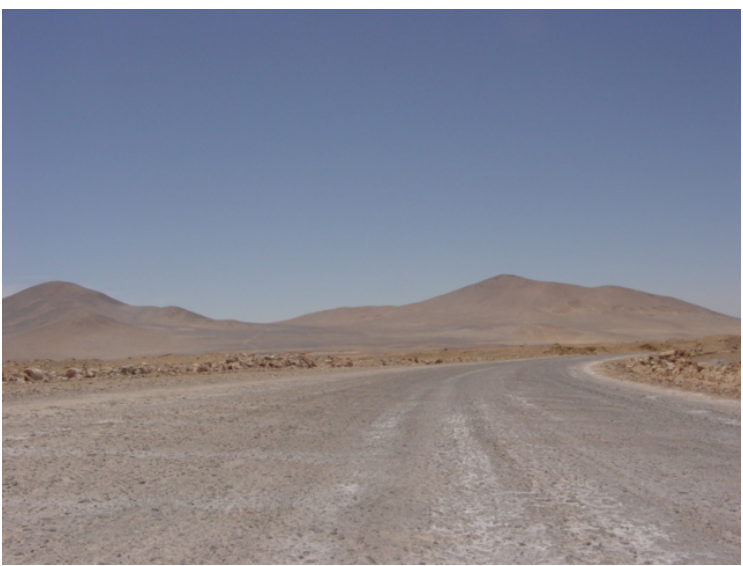

Vista parcial zona de curvas de la Ruta B-55.

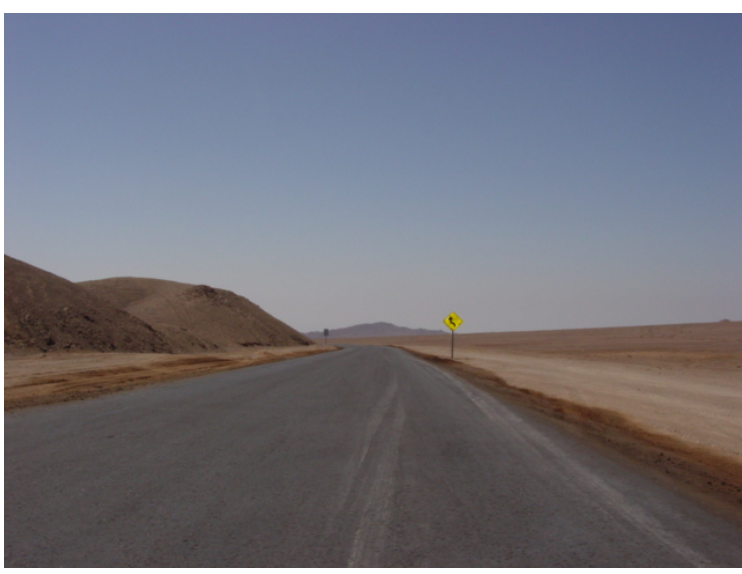

Vista parcial zona de curvas de la Ruta B- 400.

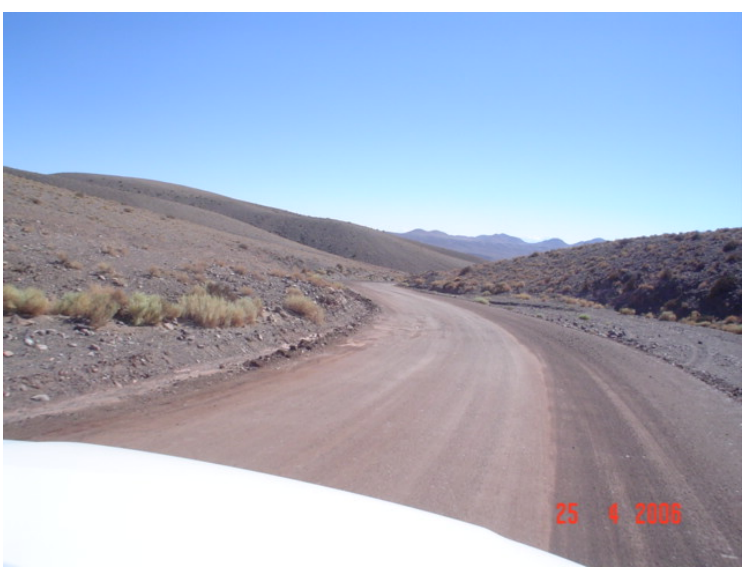

Vista parcial zona de curvas de la Ruta B- 207.

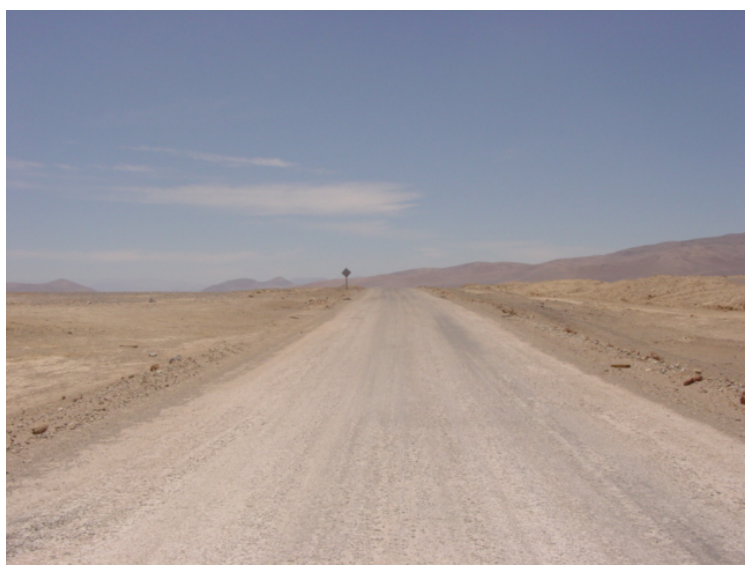

Vista parcial zona de rectas de la Ruta B-55.

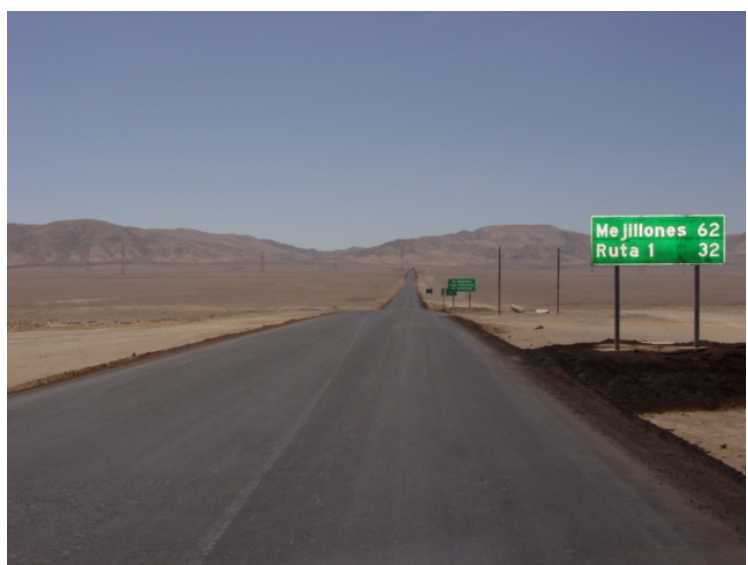

Vista parcial zona de rectas de la Ruta B-400.

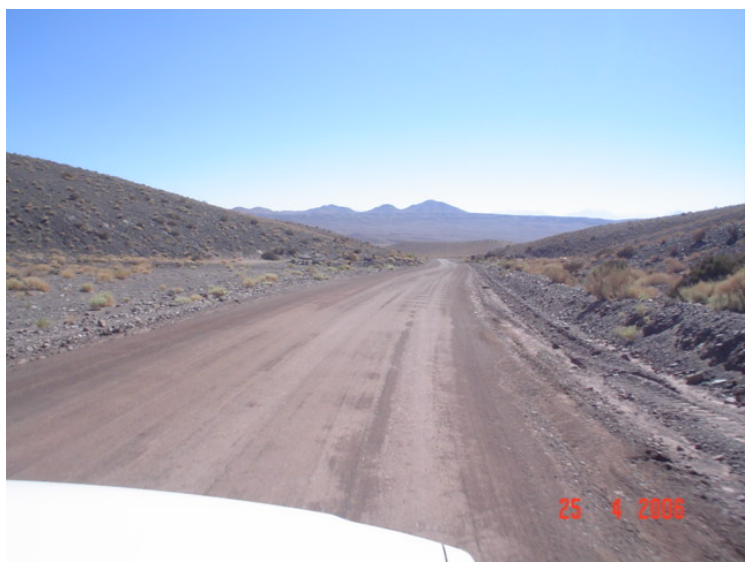

Vista parcial zona de rectas de la Ruta B-207. 


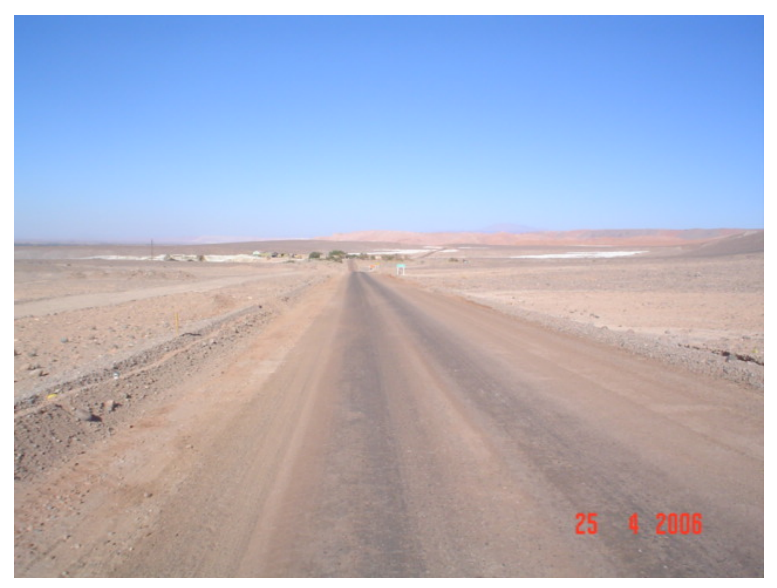

Vista parcial zona de rectas de la Ruta B- 207.

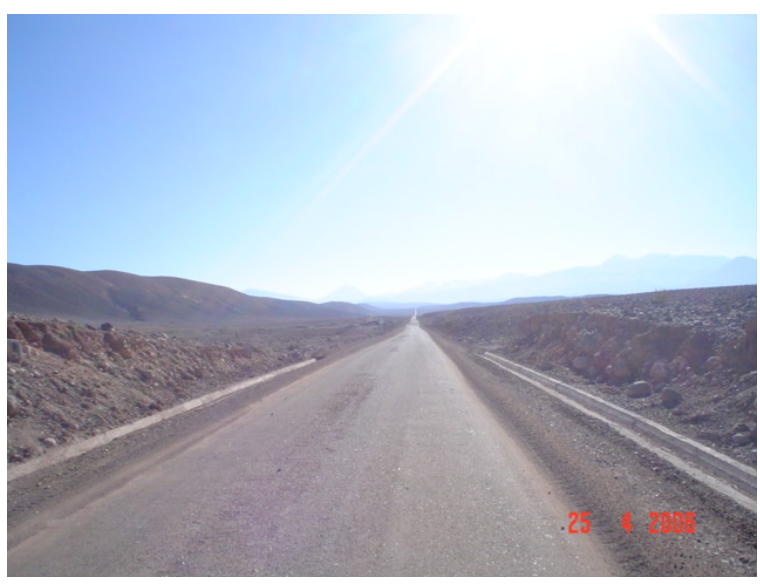

Vista parcial zona de rectas de la Ruta 21-Ch.

2. Equipo de medición de índice de regularidad internacional - IRI

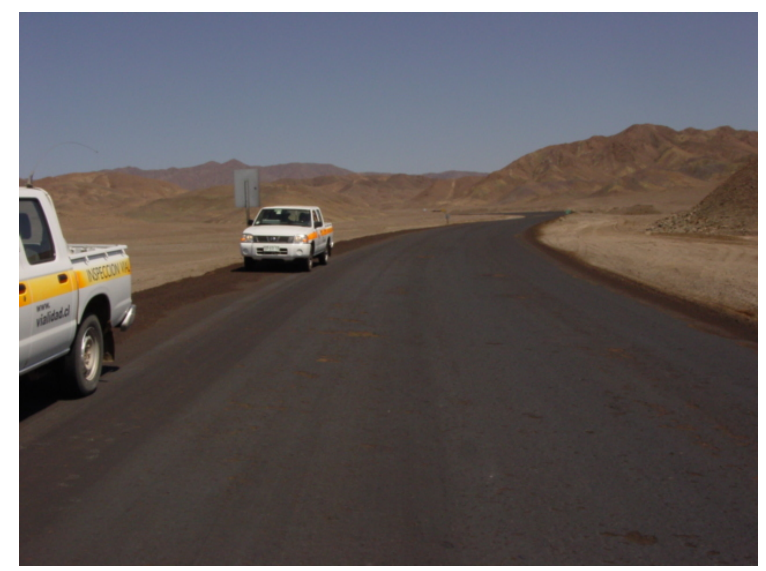

Equipo móvil de medición de la Dirección Nacional de Vialidad.

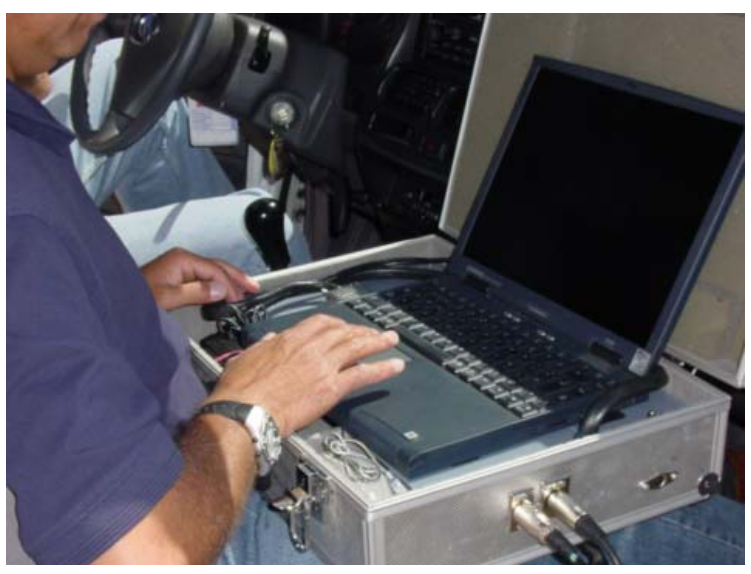

Computador conectado al acelerómetro y al odómetro del vehículo.

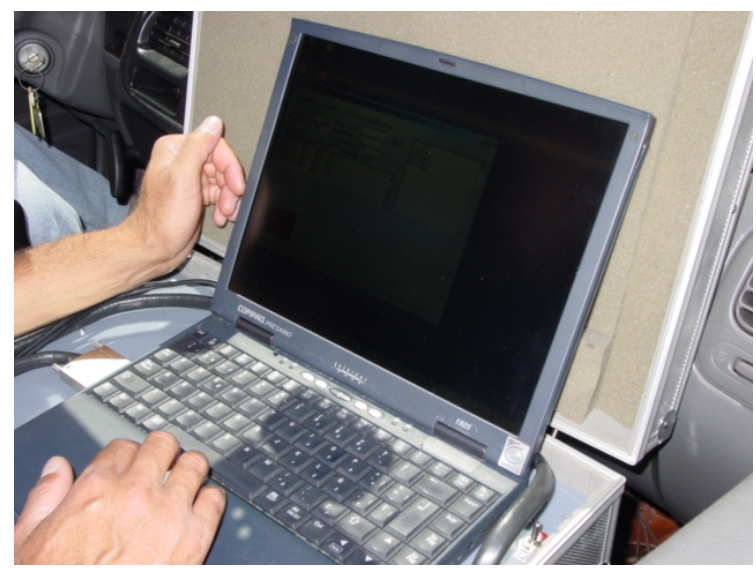

Computador equipado con software de medición.

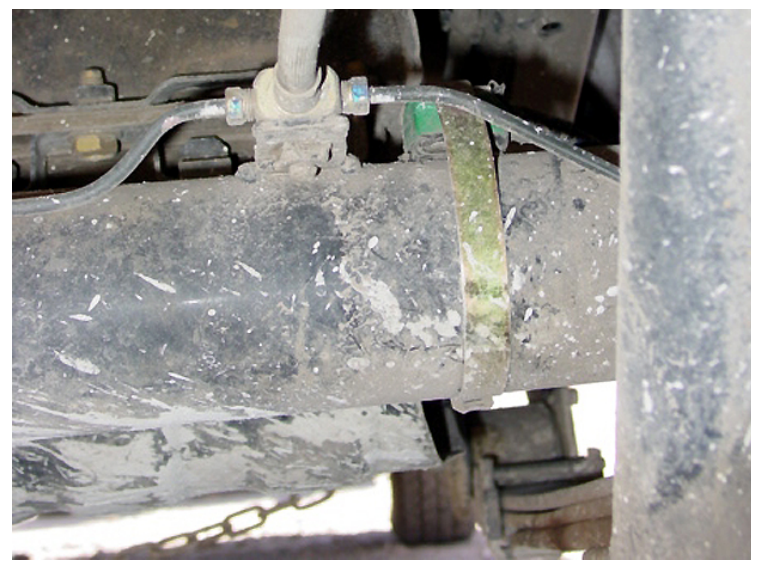

Acelerómetro instalado al eje trasero del vehículo. 


\section{Medición de Pérdida de Material por método de calicatas}

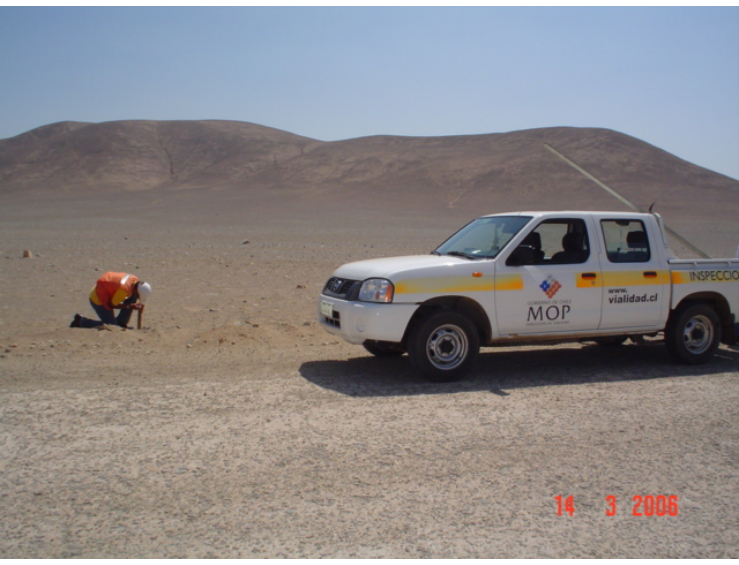

Control de estaca en borde del camino.

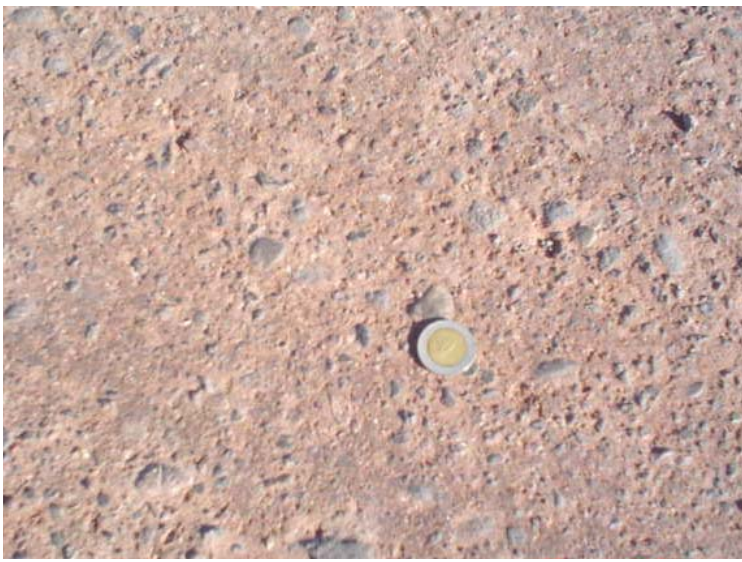

Textura superficial de las capas de rodado estabilizadas con bischofita.

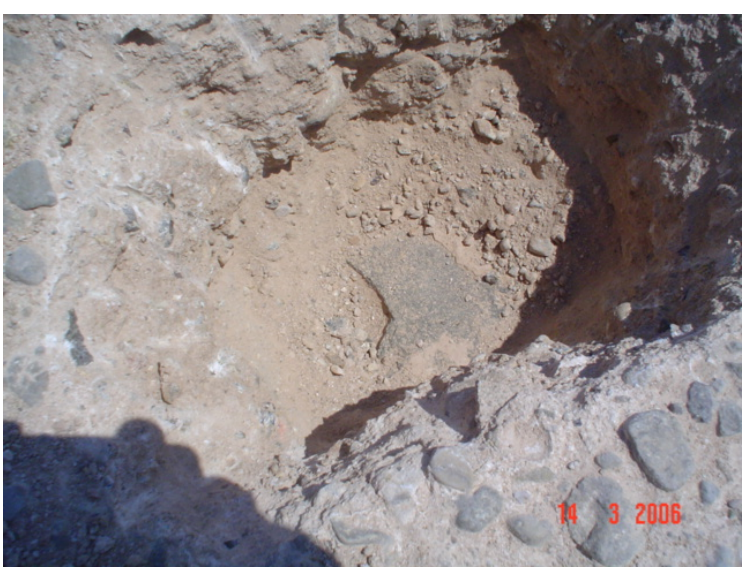

Calicata dispuesta medición de pérdida de material.

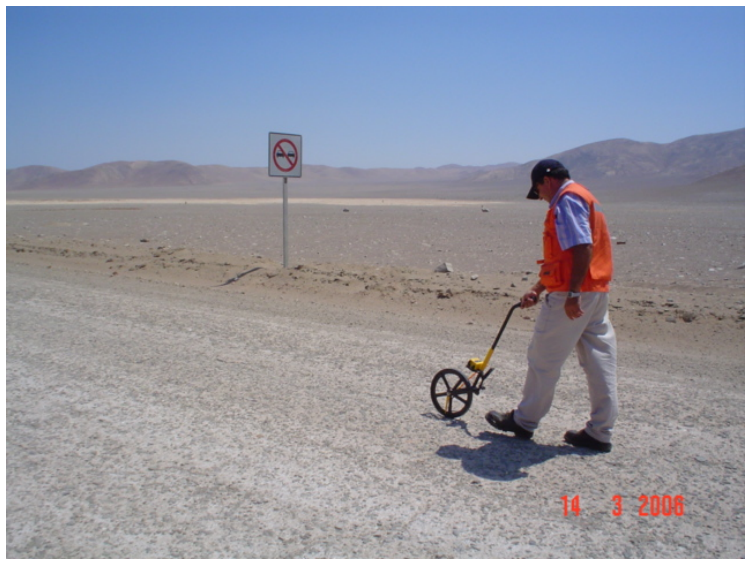

Medición con odómetro de precisión, tolerancia de $<0,02 \%$.

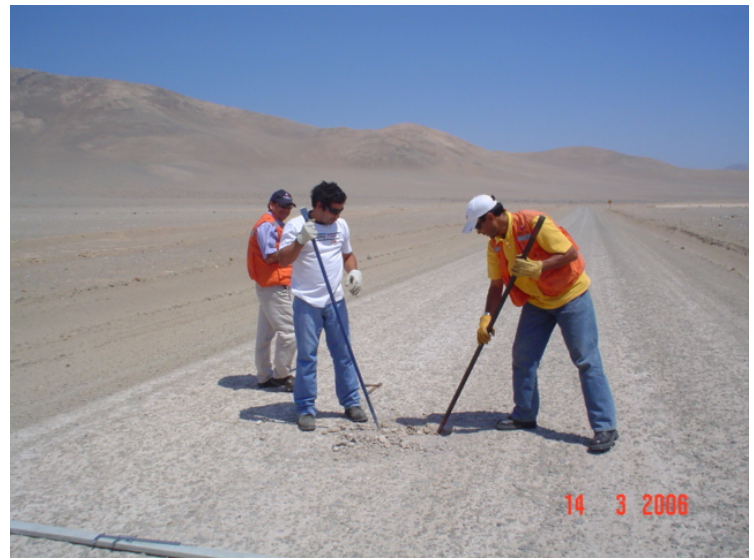

Apertura de calicatas con chuzo y pala.

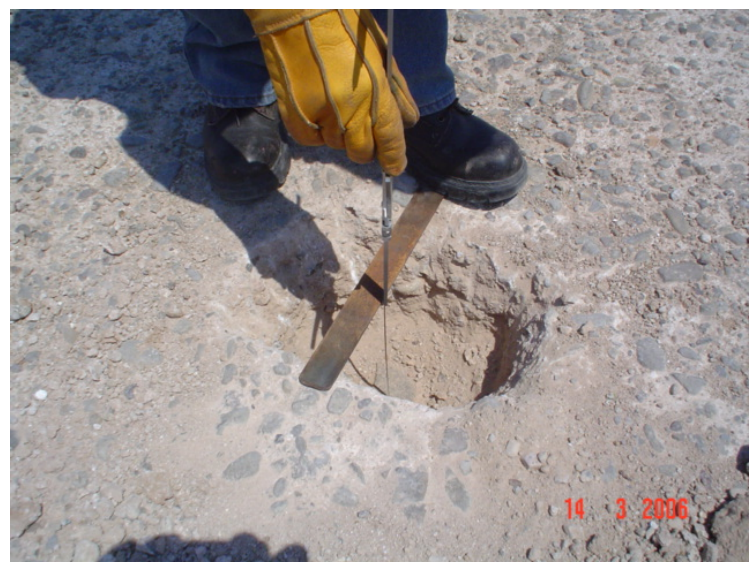

Medición de espesor remanente de las capas de rodado con piedemetro. 


\section{Imágenes varias}

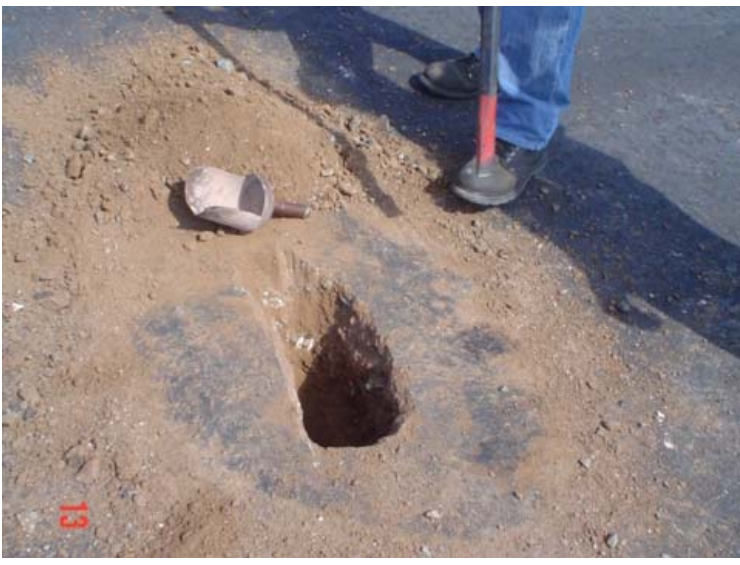

Deterioro de la capa de rodado por apertura de calicatas.

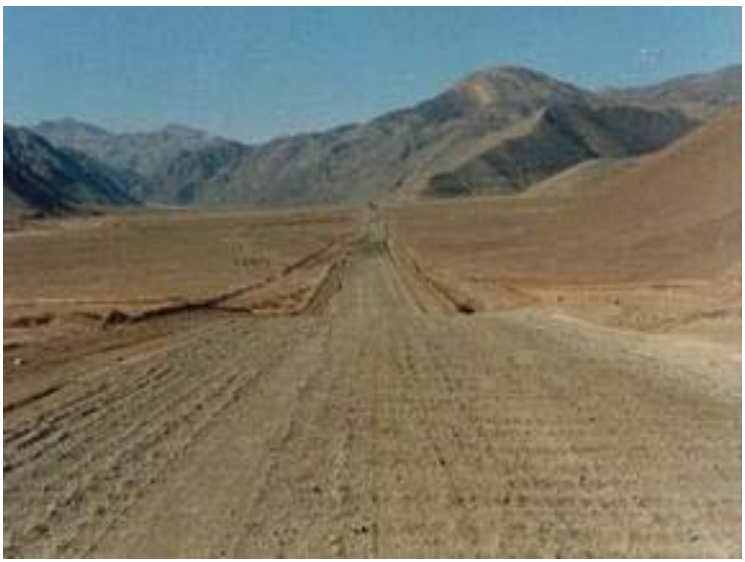

Calamina (ondulaciones) típica de los caminos no pavimentados del norte de Chile

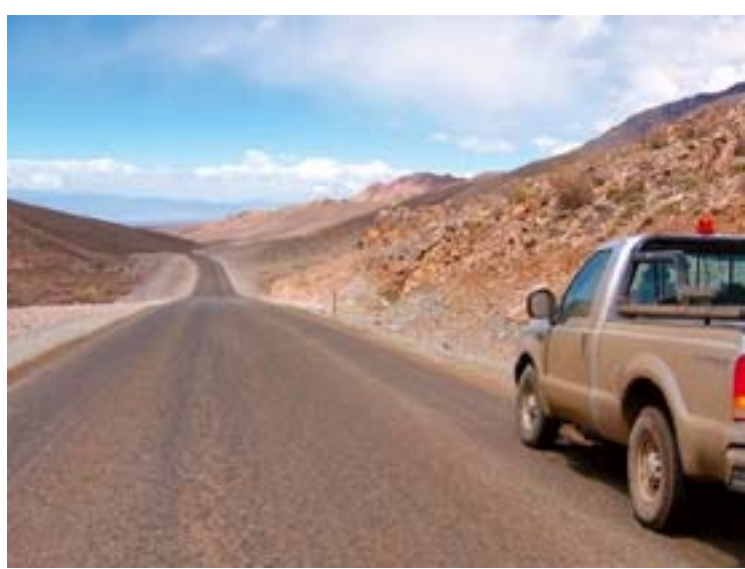

Acceso proyecto Veladero tratado con bischofita. Provincia de San Juan, Argentina.

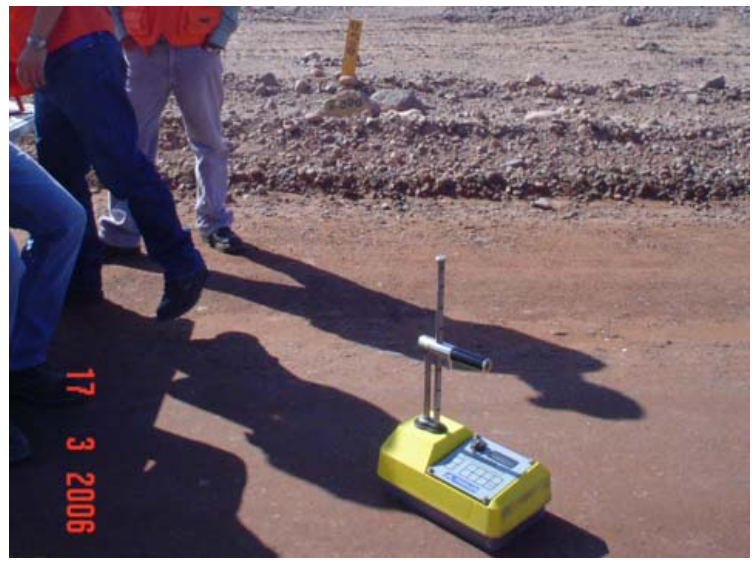

Medición in-situ de la densidad de la capa granular con densitómetro nuclear.

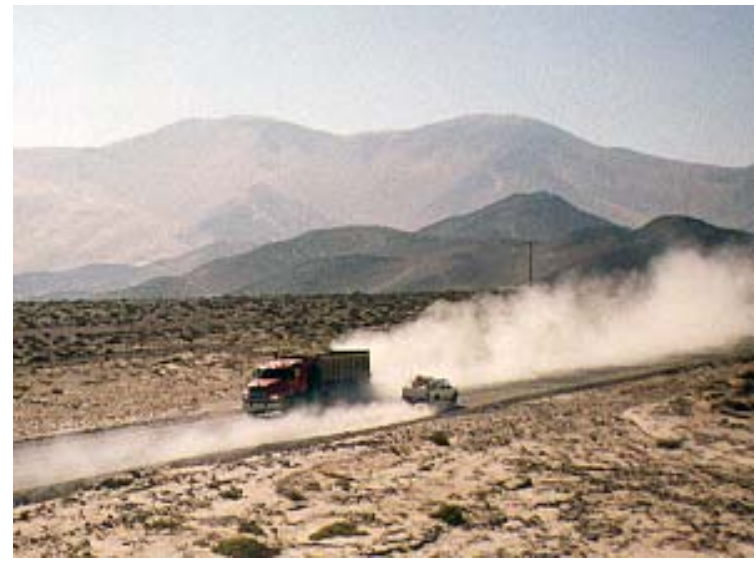

Caminos no incluidos en programa "Caminos Básicos 5.000" - Contaminación ambiental

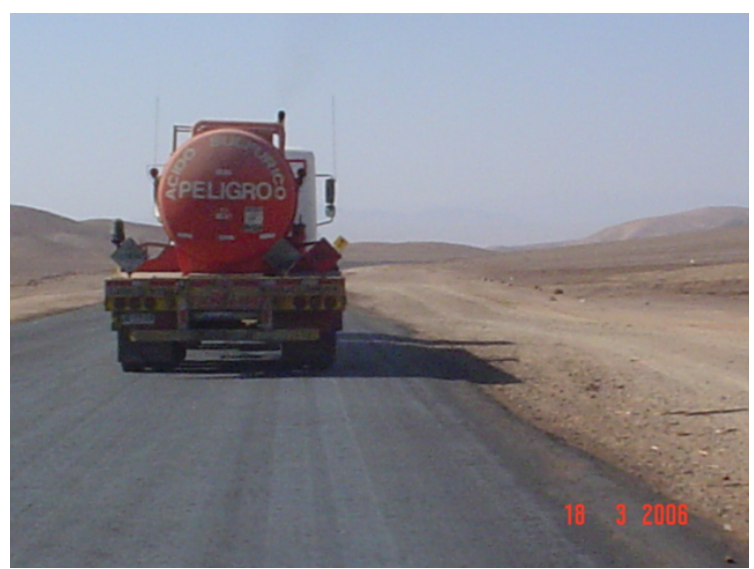

Caminos mineros en la Región de Antofagasta tratados con bischofita 